
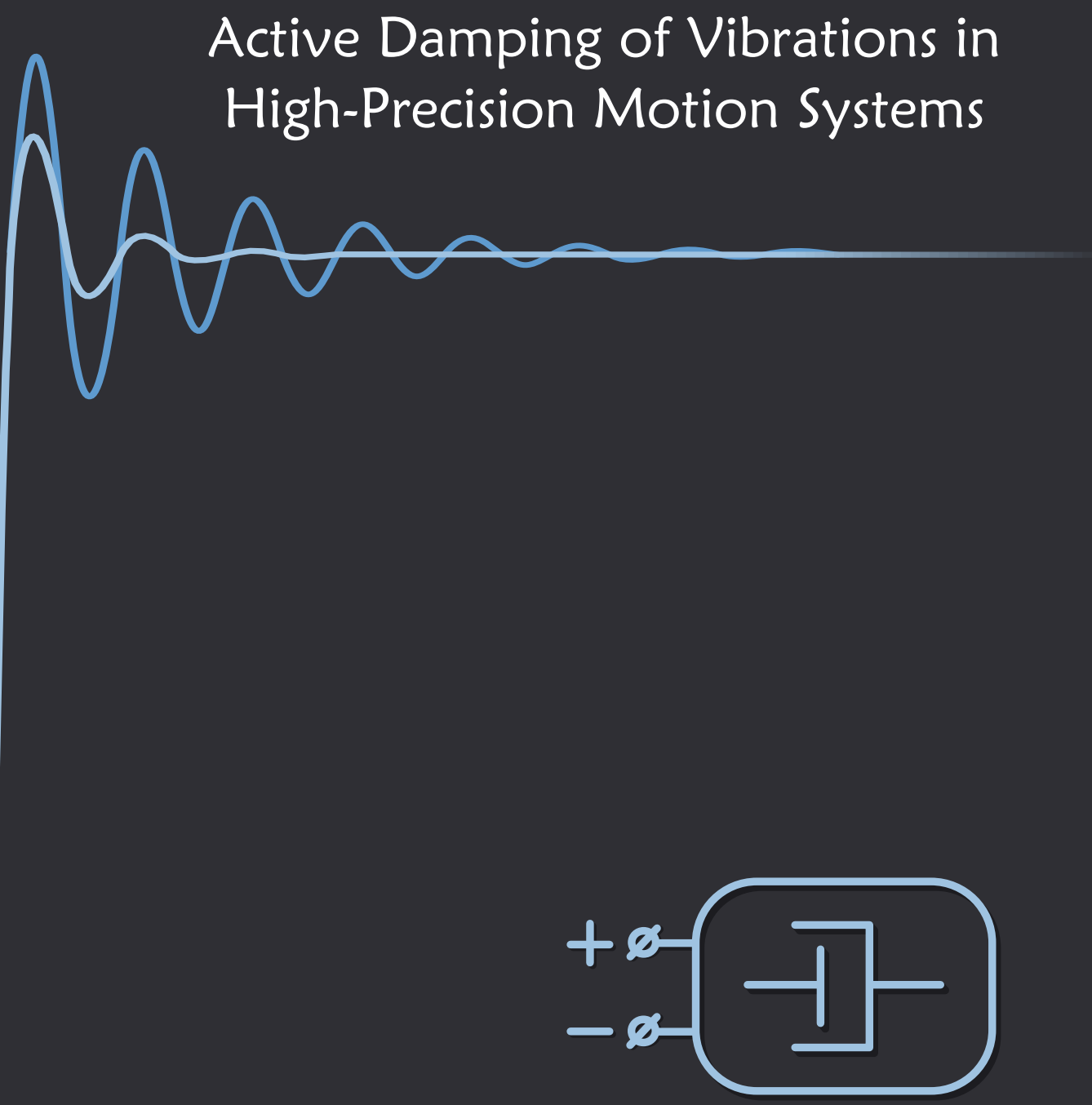

Bayan Babakhani 


\section{Propositions}

Bayan Babakhani

28-11-2012

\section{Active Damping of Vibrations in High-Precision Motion Systems}

1. To obtain optimal performance in a motion system, the AVC must be implemented in a collocated manner.

2. The use of engineering tools such as Bode plots and pole-zero plots in analysis, can help to obtain insight in the dynamics of the system, which can easily be lost in mathematical computations.

3. The mandatory Dutch summary in technical theses is redundant since it leads to texts that are even difficult to understand for Dutch peers.

4. High concentration of grants for hot topics limits the diversity of research topics, which is as necessary for the development of science as biodiversity is for the evolution.

5. The current valuation of publications leads to 'missing the science for the papers'.

6. The growing number of overlapping but independent university studies is at odds with the idea of academic training rather than vocational training.

7. The current academic training that is mainly focused on training good scientists rather than also training good educators, hinders the education of the future scientists.

8. PhD comics are funny because they are so true, and that is actually not funny (www.phdcomics.com).

9. The complexity of grey areas is due to the personal black and white borders people define for it.

10. To compromise is to give up the dream of excellence. 
Active Damping of Vibrations in High-Precision Motion Systems 
Graduation committee:

Chairperson \& Secr.: prof. dr. ir. A. J. Mouthaan Supervisor: Assistant Supervisor:

Referee:

Members: prof. dr. ir. J. van Amerongen dr. ir. T. J. A. de Vries dr. ir. J. Holterman prof. dr. ir. A. de Boer prof. dr. ir. J. L. Herder prof. dr. ir. R. H. Munnig Schmidt prof. dr. ir. H. Butler
Univ. of Twente Univ. of Twente Univ. of Twente Imotec b.v. Univ. of Twente Univ. of Twente Delft Univ. of Tech. Eindhoven Univ. of Tech.

The research described in this thesis has been conducted at the Robotics and Mechatronics group, Department of Electrical Engineering, Mathematics, and Computer Science at the University of Twente.

This research is part of the research program of the Dutch Institute of Systems and Control (DISC). The author has successfully completed the educational program of the Graduate School DISC.

The authors gratefully acknowledge the support of the Smart Mix Program of the Netherlands Ministry of Economic Affairs and the Netherlands Ministry of Education, Culture and Science.

ISBN 978-90-365-3464-2

DOI 10.3990/1.9789036534642

Copyright (c) 2012 by B. Babakhani, Enschede, The Netherlands

I, the copyright holder of this work, hereby release it into the public domain. This applies worldwide. In case this is not legally possible, I grant any entity the right to use this work for any purpose, without any conditions, unless such conditions are required by law.

Printed by Wöhrmann Print Service, Zutphen, The Netherlands. 


\title{
ACTIVE DAMPING OF VIBRATIONS IN HIGH-PRECISION MOTION SYSTEMS
}

\author{
PROEFSCHRIFT
}

ter verkrijging van

de graad van doctor aan de Universiteit Twente, op gezag van de rector magnificus,

prof. dr. H. Brinksma,

volgens besluit van het College voor Promoties

in het openbaar te verdedigen

op woensdag 28 november 2012 om 14.45 uur

door

Bayan Babakhani

geboren op 26 december 1982

te Teheran, Iran 
Dit proefschrift is goedgekeurd door:

Prof. dr. ir. J. van Amerongen, promotor Dr. ir.T.J.A. de Vries, assistent-promotor 


\section{Summary}

Technology advancements feed the need for ever faster and more accurate industrial machines. Vibration is a significant source of inaccuracy of such machines. These vibrations are either caused by external disturbances, or result from structural compliances. Moreover, a light-weight design in favor of the speed, and avoiding the use of energy-dissipating materials from the structure to omit any source of inaccuracy, contribute to a low structural damping. Consequently, vibrations manifest themselves as badly damped oscillations in the end-effector response. Hence, the performance is degraded by a long settling time and the corresponding uncertainty in the position of the end effector.

Speed and accuracy in motion systems can be attained by implementing a high-bandwidth motion controller. However, the resonances in the plant transfer impose a limit on the achievable bandwidth of such a controller.

An example of a plant with structural resonances is a machine fitted with linear actuators. The guideways on such actuators have a certain compliance. This compliance causes rotational vibrations of the end effector around the guideway: the so-called 'Rocking mode'.

The goal of this research is to investigate the addition of damping to the rotational vibration mode of a linearly actuated motion system to

- achieve a shorter settling time in the transient response of the plant to a commanded motion

- increase the achievable closed-loop motion-control bandwidth

This thesis starts by showing the influence of damping on the stability of motion systems, for P(I)D-type motion controllers. A root-locus analysis validates the increase of the achievable bandwidth resulting from the increase in modal damping, to various extents for different types of high-order modes.

Furthermore, a set of guidelines is presented that can be used for a mechatronic design of an active-damping loop. It is shown that collocated active damping increases the damping of both the poles and the zeros of the motioncontrol loop. This allows for a higher increase of motion-control bandwidth than 
when the same actuator is used in both the motion-control loop and the activedamping loop. The chosen control algorithm for collocated active damping is integral force feedback. This combination results in a robustly stable closed-loop system. The effect of active damping on the end-effector dynamics of a motion system is analyzed extensively in simulation.

The practical implementation of active damping in a setup that suffers from a rotational vibration mode showed the performance improvement by damping in the transient behavior of the plant. The increase of the bandwidth as a result of active damping could not be demonstrated using the current test setup, because of the relatively high damping that is already present in the setup. 


\section{Samenvatting}

Technologische vooruitgang zorgt voor een behoefte aan steeds snellere en nauwkeurigere industriële machines. Trillingen vormen in zulke machines een belangrijke bron van onnauwkeurigheid. Deze trillingen kunnen worden veroorzaakt door externe stoorbronnen of zijn het resultaat van slapheden in de mechanische structuur. Bovendien, een lichtgewicht ontwerp ten behoeve van de snelheid, en het vermijden van energie-dissiperende materialen om zo elke bron van onnauwkeurigheid te elimineren, dragen bij aan de lage demping van de mechanische structuur. Daardoor manfesteren trillingen zich als slecht gedempte oscillaties in de eind-effector responsie. De prestatie wordt verslechterd door een lange indicatietijd met de bijbehorende onzekerheid in de positie van de eindeffector als gevolg.

De combinatie van snelheid en nauwkeurigheid in bewegende systemen kan worden bereikt door gebruik te maken van een regelaar met een hoge bandbreedte. De resonanties in de overdracht van het systeem begrenzen echter de haalbare bandbreedte van de regelaar.

Een voorbeeld van een systeem met mechanische resonanties is een machine uitgevoerd met lineaire actuatoren. De geleidingen van deze actuatoren hebben een zekere compliantie. Deze compliantie zorgt voor rotatietrillingen van de eind-effector rondom de geleiding, de zogeheten wiebelmode ("Rocking mode").

Het doel van dit onderzoek is om demping toe te voegen aan de rotatietrillingsmode van een met lineaire actuatoren aangestuurd systeem, om zo het volgende te bereiken:

- een kortere indicatietijd in de natuurlijke (transiënt) responsie van het systeem op een referentie verandering

- de haalbare bandbeedte van het gesloten regelsysteem te verhogen

In dit proefschrift wordt eerst de invloed van demping op de stabiliteit van bewegende systemen bekeken voor regelaars van het type P(I)D. Een poolbaan analyse valideert de verhoging van de haalbare bandbreedte die het resultaat is 
van verhoging van de modale demping voor verschillende types van hoge orde modi.

Verder wordt een set vuistregels gepresenteerd die kunnen worden gebruikt bij het mechatronisch ontwerp van een actieve demping regellus. Hierbij is aangetoond dat gecolloceerde actieve demping de demping van zowel de polen als de nulpunten van de bewegingsregellus vergroot. Dit maakt een grotere verhoging van de bandbreedte van de bewegingsregellus mogelijk ten opzichte van de situatie waarin dezelfde actuator wordt gebruikt in zowel de bewegingsregellus als de actieve dempingslus. Het gekozen regelalgoritme voor gecolloceerde actieve demping is geïntegreerde kracht terugkoppeling. Deze combinatie resulteert in robuuste stabiliteit van het gesloten systeem. Het effect van actieve demping op de eind-effector dynamica van een bewegend systeem is uitgebreid geanalyseerd door middel van simulaties.

De in de praktijk uitgevoerde implementatie van actieve demping in een opstelling die last heeft van een rotatietrillingsmode heeft aangetoond dat demping van het natuurlijke gedrag van het systeem tot prestatieverbetering leidt. De met actieve demping verkregen verhoging van de haalbare bandbreedte kon op de huidige opstelling niet worden gedemonstreerd. Dit komt doordat er in de opstelling reeds een relatief hoge demping aanwezig is. 


\section{Contents}

Summary $i$

Samenvatting

1. Introduction 1

1.1 Research objectives . . . . . . . . . . . . . . . . . . . . 3

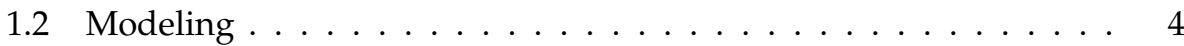

1.3 Active damping . . . . . . . . . . . . . . . . . . 5

1.4 Thesis outline . . . . . . . . . . . . . . . . . 8

2 Stability of P(I)D-controlled motion systems 11

2.1 Introduction . . . . . . . . . . . . . . . . . 12

2.2 Dynamic plant model . . . . . . . . . . . . . . . . . . . . . . . . . . . . . . . . . . . .

2.3 Motion controllen . . . . . . . . . . . . . . . . . . . . 17

2.4 Stability analysis . . . . . . . . . . . . . . . . . . . . . . . . . . . . . . . . . . . . . . .

2.4 Method . . . . . . . . . . . . . . . . . . . . . . . . . . . . . . . . . . .

2.4 .2 Stability; $\omega_{c}$ versus $\alpha$ versus $\xi \ldots \ldots$. . . . . . . . . 19

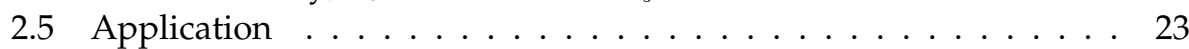

2.6 Conclusion . . . . . . . . . . . . . . . . . . . 27

3 Collocated vs Noncollocated Active Damping 29

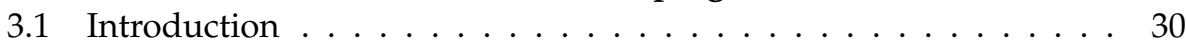

3.2 Model of the plant . . . . . . . . . . . . . . . . . . . . . . . . . . . . . . . . . . .

3.3 Active damping . . . . . . . . . . . . . . . . . . . . . . . . . . . . . . . . . . . . . . . 34

B.3.1 Collocated AVD . . . . . . . . . . . . . . . . . 35

3.3 .2 Noncollocated AVO . . . . . . . . . . . . . . . 41

3.4 Results; AVC in motion control closed loop . . . . . . . . . . . . . . 43

3.5 Simulated step responses . . . . . . . . . . . . . . . . . . . . . . . . . . . . . . . 45

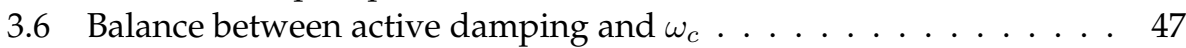

B.7 Conclusion . . . . . . . . . . . . . . . . . . . . . 48 
4 Experimental Evaluation of Active Damping 51

4.1 Introduction . . . . . . . . . . . . . . 52

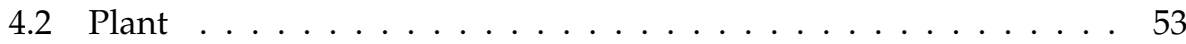

4.2 .1 Pant model . . . . . . . . . . . . . . . . 53

4.2 .2 Rocking mode demonstrator . . . . . . . . . . . . . . 54

4.2 .3 Motion controlloop . . . . . . . . . . . . . . . . 56

4.3 Active damping . . . . . . . . . . . . . . . . . . . . . . . . . . . . . . . . . . . 57

4.3 .1 Active vibration control . . . . . . . . . . . . . . . . . . . . . . . . . . 58

4.3 .2 Active damping unit . . . . . . . . . . . . . . . . . . 59

4.3 .3 Static response compensation . . . . . . . . . . . . . . 66

4.4 Measurements . . . . . . . . . . . . . . . . . . . . 68

4.5 Conclusions . . . . . . . . . . . . . . . 71

5 Off-axis Modal Active Vibration Control 73

5.1 Introduction . . . . . . . . . . . . . . . . 74

5.2 Modal model of vibrations . . . . . . . . . . . . . . . . 75

5.3 3D dynamical model of the plant . . . . . . . . . . . . . . . . . 76

5.3 .1 Rigid-body motion . . . . . . . . . . . . . . . . 77

5.3 .2 The plant model using the screw theory . . . . . . . . . . 78

5.4 Active damping . . . . . . . . . . . . . . . . . . 83

5.4 .1 Active vibration controllen . . . . . . . . . . . . . . . 83

5.4 .2 ADU and end effector interaction . . . . . . . . . . . . 84

5.5 Simulations . . . . . . . . . . . . . . . . . . . . . . . . . . . . . . . . . . 87

5.6 Conclusions . . . . . . . . . . . . . . . . . . . 89

6 Loss of Collocation 91

6.1 Introduction . . . . . . . . . . . . . . . . . . 92

6.2 Collocated active damping . . . . . . . . . . . . . . . . . . . . . . . . . . . . . . . . . . . . . . .

6.2 .1 Leaking integral force feedback . . . . . . . . . . . . . . . . . . . . . . . . . . . . . . .

6.2 .2 Active damping loop . . . . . . . . . . . . . . . . . . . . 94

6.2 .3 Stability and higher-order modes . . . . . . . . . . . . . . . 96

6.3 Stability analysis . . . . . . . . . . . . . . . . . . . . . . . . . . . . . . . . . . . . .

6.3 .1 Active damper design . . . . . . . . . . . . . . . . . . . . . . . . . . . . . . . . . .

6.3 .2 Tuning active damping controller . . . . . . . . . . . . 102

6.4 Conclusions . . . . . . . . . . . . . . . . . 103

6.5 Discussion . . . . . . . . . . . . . . . . . . . 104

7 Conclusions and Recommendations $\quad 107$

Z. Conclusions . . . . . . . . . . . . . . . . . . . . . . . 107

Z2 Recommendations ....................... . . 110

A Detailed mechanical design of the ADU 113 
\begin{tabular}{ll}
\hline List of publications & 121
\end{tabular}

\begin{tabular}{ll}
\hline Bibliography & 123
\end{tabular}

\begin{tabular}{ll}
\hline Acknowledgment & 131
\end{tabular}

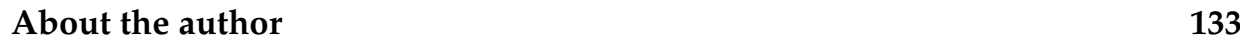




\section{Chapter 1}

\section{Introduction}

Precision machines are designed to be fast and accurate. Therefore, effort is made to omit any source of inaccuracy from the structural design of the plant. Reducing friction, using constructions with a high stiffness, and making lightweight moving parts are among the measures that can be taken. In striving for lightness and stiffness, the damping design of the structure is often overlooked. These actions lead to nearly undamped high-order resonance modes in the transfer of the plant.

The vibrations present in these machines are usually the bottleneck for achieving the desired performance. These vibrations may be caused by external disturbances, such as floor vibrations, but they also may result from structural compliances. Such vibrations appear as badly damped oscillations in the endeffector response. Hence the performance is degraded by a long settling time and the corresponding uncertainty in the position of the end effector.

Speed and accuracy in motion systems can be achieved by implementing a high-bandwidth motion controller. However, the resonances in the plant transfer impose a limit on the bandwidth of such a controller. So, to obtain a good performance, it is important to control these vibrations such that their effect on the system behavior is reduced, or if possible, even eliminated.

If the vibrations are forced and the disturbance source and the end effector are located at different places, vibration isolation can be implemented. The goal of isolation techniques is to minimize the power transfer between the disturbance source and the performance metric (MacMartin 1995, Zou \& Slotine 2005).

When the vibrations are caused by the resonance modes of the structure, and are hence distributed in nature, adding damping is profitable. The aim is then to maximize the power dissipation resulting in the damping of the resonances (Breumont 1997, Bolterman 20012, Vervoordeldonk et al, 20066).

Increasing the damping can be realized by the so-called passive damping, which means taking mechanical measures that lead to a higher dissipation in 

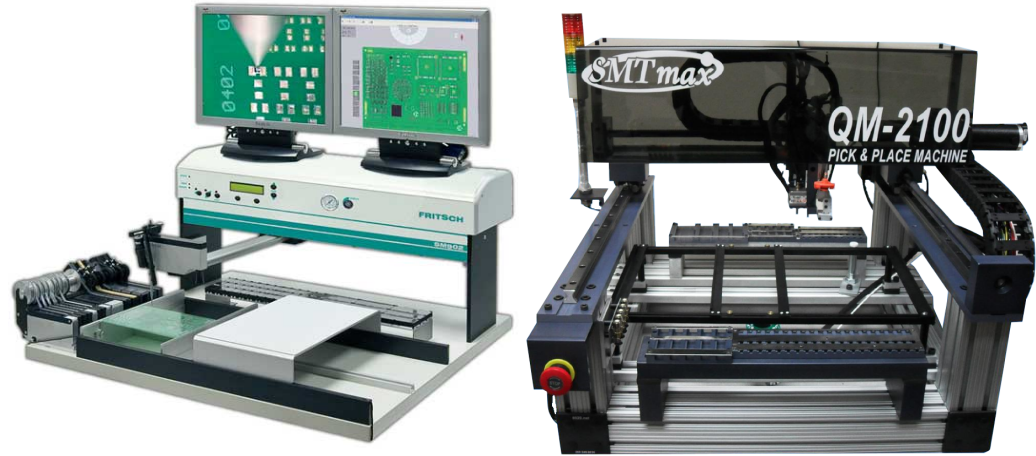

(a) SM902; semi-automatic pick-

(b) QM210011; automatic pick-andand-place machine. Photo courplace machine. Photo courtesy of tesy of Fritsch ( $\mathrm{mbH}$ (2012) Omxie/SMTmax Corp. (DIT)

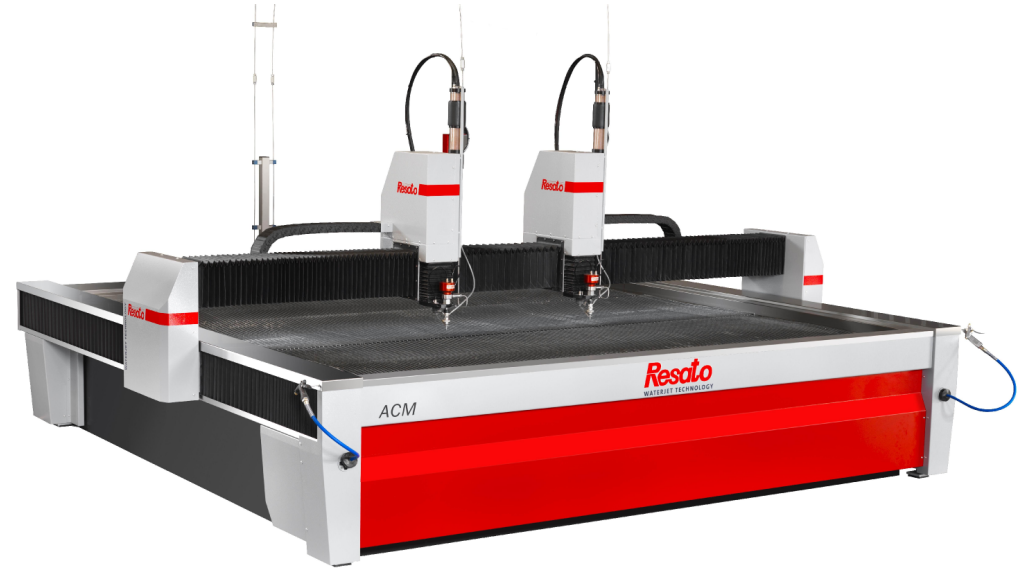

(c) ACM; waterjet cutting machine. Photo courtesy of Resato International b.v. (2012)

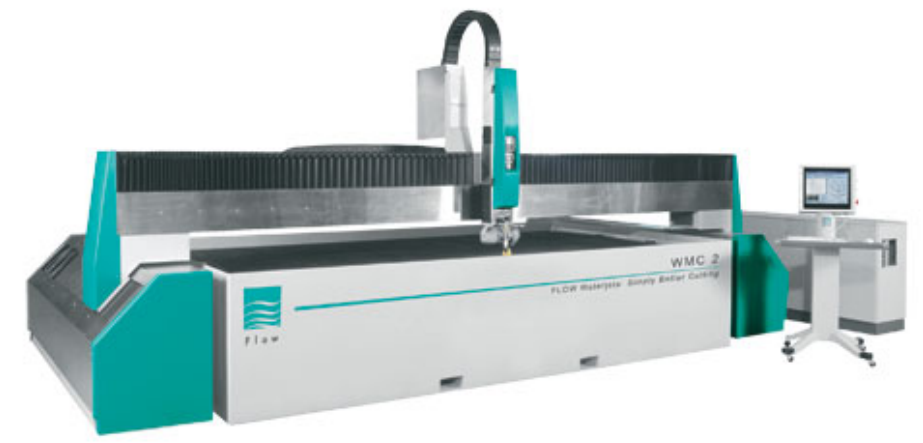

(d) Flow WMC2; waterjet machining center. Photo courtesy of Flow International Corporation (2012)

Figure 1.1: A few examples of industrial high-precision machines fitted with linear actuators. 
the plant. This can, for instance, be done by modifying the design of the structure geometry (Keane \& Bright 1996), or embedding damping material in the structure (Mead 1998, Jiang \& Miles 1999). However, passive damping can interfere with the overall system dynamics. In addition, the performance of the damping material might be subject to fatigue or sensitive to environmental factors, such as temperature.

When passive measures are not sufficient, cannot be realized, or are too costly, an intelligently designed mechatronic solution, termed active damping, can be used to add damping to the undesired vibrations. Active damping can be applied to a limited frequency band, achieving a higher damping for the target vibration mode(s).

\subsection{Research objectives}

The focus of this research is on industrial high-precision machines fitted with linear actuators, such as pick-and-place machines and cutting machines, a few examples of which are shown in Figure 1.7. The guideway on the linear actuator of such machines has a certain compliance. This compliance causes rotational vibrations of the end effector around the guideway: the so-called 'rocking mode'. Figure 1.2 shows a sketch of this situation.

When the structural damping in such machines is low, the rocking mode is manifested as oscillatory behavior of the end effector. In addition, the rocking mode makes the implementation of a motion controller more challenging. To suppress the high-frequency disturbances, it is desirable to equip the motion controller with a low-pass filter. However, implementing such a controller for a plant suffering from the rocking mode is only possible in combination with a low gain and low bandwidth. Active damping (see Figure [1.31) allows for the increase of the system bandwidth, without the danger of instability. In turn, this allows for a higher integral gain in the motion control algorithm.

The goal of this research is to investigate the addition of damping to the rotational vibration mode of a linearly actuated motion system to

- achieve a shorter settling time in the transient response of the plant to a commanded motion

- increase the achievable closed-loop motion-control bandwidth to obtain an improved performance 

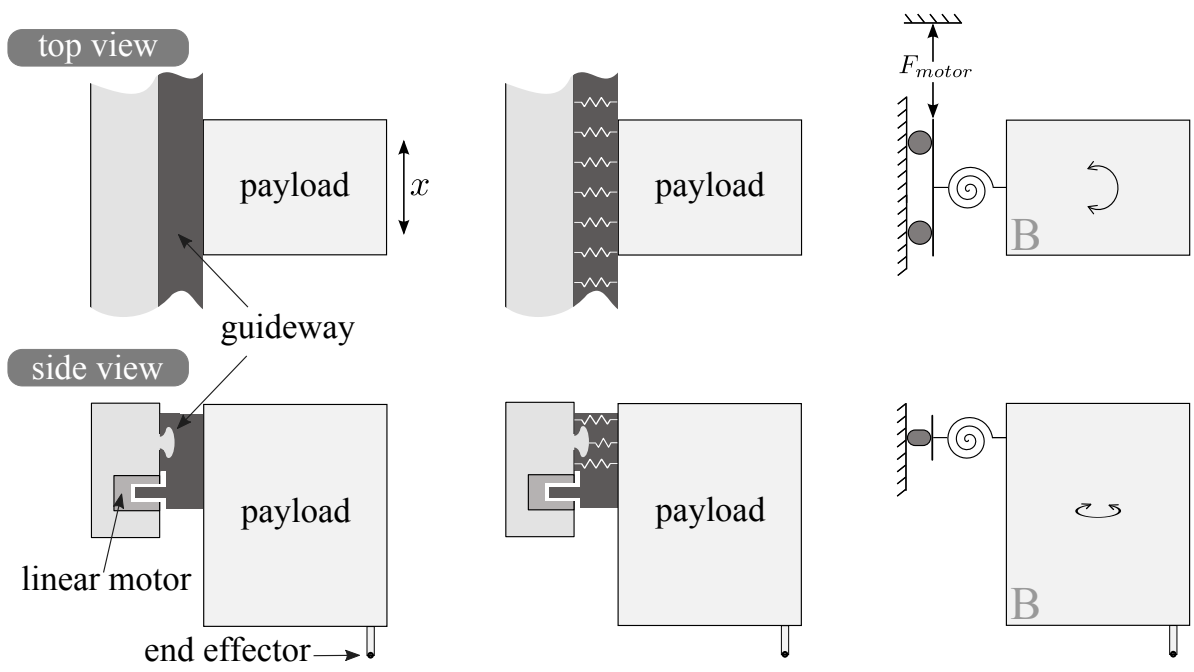

\section{side view}
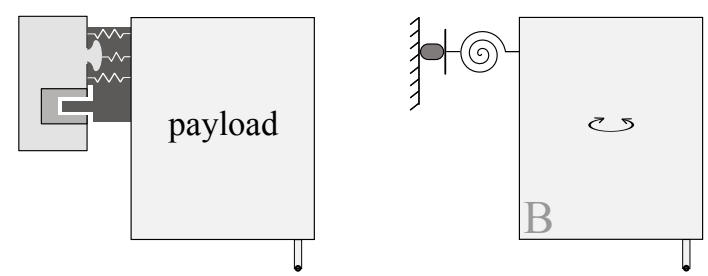

Figure 1.2: Schematic top view (top three) and side view (bottom three) of the rocking mode in a machine fitted with linear actuators. The compliances in the guideway (shown symbolically in the middle) result in rotational vibration of the payload, as shown on the right side. Note that the rotational-spring icon shown in the plot is symbolic and does not represent the rotational axis.

\section{top view}

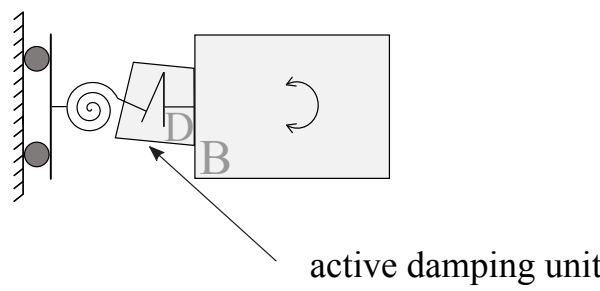

\section{side view}

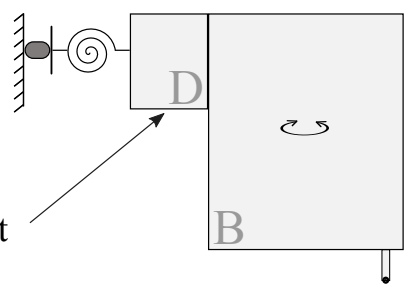

Figure 1.3: Schematic plot of the active damping in a machine suffering from the rocking mode.

\subsection{Modeling}

Analyzing the system behavior, designing a controller, and simulating the effect of any modification of the plant before the actual implementation, requires a competent model of the system. Making such a model can be done using various modeling techniques. In each chapter, a short description of both the modeling 
technique and the resulting models that are used to target the specific problem which that chapter deals with, is given. Here, a short overview will be given of the theories and the modeling techniques that are used in this thesis. The reader is referred to Meirovitch (1986), de Silva (2000), Karnopp et al. (2006), and Duindam et al. (2009) for more detailed information.

The dynamics of a motion system can be modeled as a set of differential equations of motion, derived using the Newton's second law. Here, the system is considered to have a set of lumped parameters modeled as masses, stiffnesses and dampers. These equations are generally coupled. For vibration control purposes, it is convenient to decouple the dynamic model of the system. This can be done using the orthogonality property of the modal vectors belonging to the eigenvalues of the structure.

When the relevant dynamics of a structure cannot be modeled adequately by lumped parameters, distributed system modeling techniques can be used, which represent the plant by partial differential equations. A distributed-parameter model of the Active Damping Unit (ADU) has been used in the detailed design phase of the mechanics of the active damper for analyzing the stresses and forces acting on the mechanism. This analysis assists in making a mechanically robust design. Also, a distributed model was used to validate the lumped-parameter model of the active damper (see Chapter 1 ).

The dynamics of the end effector and its performance are usually expressed in terms of Euclidean coordinates. Modeling the interaction between the active damping unit and the end effector can be performed using the screw theory. This modeling technique maps the geometry of the structure to a three-dimensional model of the plant, which can be used to transform the modal vibrations to Euclidean coordinates at the end-effector location.

\subsection{Active damping}

In literature, there are various vibration control methods available that can be chosen depending on the situation at hand. Possible consideration are the extent of the knowledge of the plant dynamics, the available signals in the control system, the parameter uncertainty, but also limitations that can apply for the mechanical implementation.

When a signal correlated to the disturbance causing vibrations is available in the control system, or the disturbances are either repetitive or predictable, feedforward control can be implemented. In effect, feedforward controllers generate a signal matching the amplitude of the disturbance, but with the opposite phase, to cancel the effect of the disturbances. Feedforward can be used for both motion control and active vibration control. A few implementations of vibration control by feedforward are the active hard mount for vibration isolation (van der Poel 2010), feedforward both with and without noncollocated 
acceleration feedback (Chen \& Tlusty 1995) and an adaptive notch filter for torsion vibration compensation in lead-screw feed drives (Zhou et al. 20(1)). Also input shaping, introduced by Singer \& Seering (1989), can be used to suppress the plant vibrations within the motion loop (Zhou \& Misawa 2005, Murphy \& Watanabe 1992, Mohamed \& Tokhi 20(14). Note that these methods either require an accurate model of the structure, or should possess learning capabilities, which can be computationally intensive. Also, they deal with the vibrations, but do not add damping to the structure.

Active damping can be realized by means of feedback control. Feedback control can be implemented in both a collocated and a noncollocated configuration. There are various control algorithms available in literature for both collocated and noncollocated feedback control. The reader is referred to Preumont (1997), Franklin et al. (2006), and Symens (20)4) for an overview of these algorithms, and Anderson \& Hagood (1994) and van Schothorst (1999) for comparisons between the performance of some of the algorithms for vibration control specifically.

Collocated control refers to the fact that the dual sensor and actuator of the control loop are physically at the same location. This configuration allows for controlling the power flow from the controller to the structure and thus enables the implementation of robustly stable control loops. Smart discs in microlithography machines (Holterman \& de Vries 2005), active truss elements and active tendons for large space structures (Preumont \& Loix 200(0)), active legs in a Stewart platform (Geng \& Haynes 1994), sensor fusion strategy on a multi-axes vibration isolation system having six collocated actuatorÜsensor pairs (Ijepkema et al. 2012), adaptive trusses for vibration isolation (Clark \& Robertshaw 1997), simultaneous piezoelectric sensing/actuation in combination with either resistive and resonant shunting (Hagood \& von Flotow 1991), or active control algorithms, applied to a cantilevered beam (Anderson \& Hagood 1994), and the hybrid approach combining low-authority wave control and highauthority modal control, implemented for a cantilever beam (Mei et al. 2001) are a few examples of collocated vibration control.

Noncollocated control requires a model of the structure. This can be resolved by using a controller that includes an implicit observer, such as $\mathrm{H}_{\infty}$ synthesis (Âström 2008) or an explicit model of the structure. Relevant works in this field are the disturbance adaptive discrete sliding mode controller for feed drives fitted with linear motors by Altintas \& Okwudire (20)8), the implementation of an $\mathrm{H}_{\infty}$ based vibration controller in a pick-and-place setup by Verscheure et al. (2006), vibration control of a flexible beam using noncollocated PZT actuators and strain gauge sensors by Manning et al. (200) ), active vibration control of a flexible beam by noncollocated acceleration feedback using both proportional and sliding mode control by Qiu et al. (20109), and integral resonant control algorithm -also known as integral force feedback- of a single-link flexible manipulator using an explicit model of the plant by Pereira et al. (20T1). Note that only the first two take 


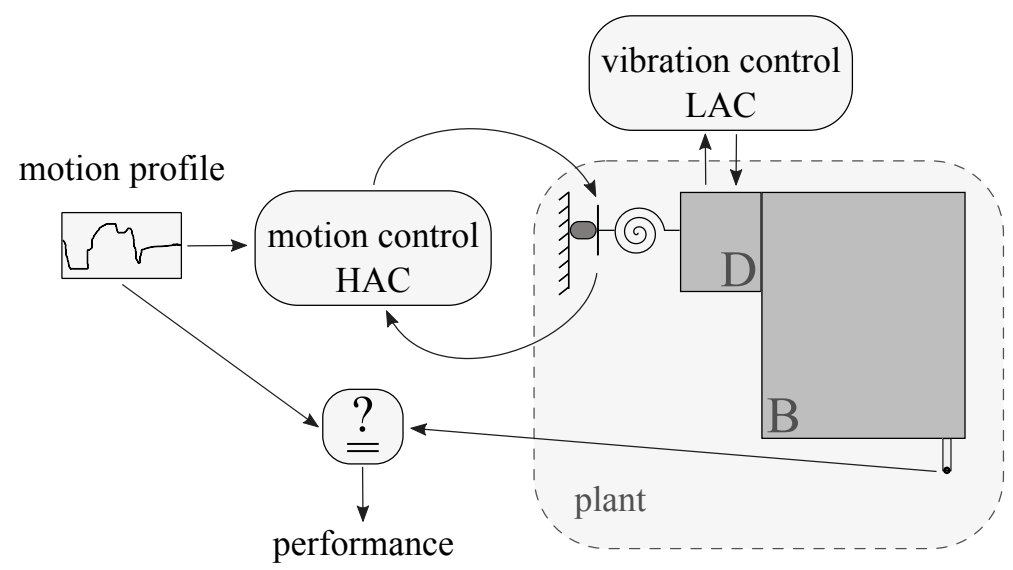

Figure 1.4: A sketch of the system showing the motion control loop (including the high-authority controller), the active damping loop (including the low-authority controller), and the performance loop.

parameter variation of the plant during operation into account.

In general, it can be stated that the more a controller is designed to suit the plant dynamics, the higher the achievable performance can be. The downside is however, the corresponding decrease of the stability robustness for modeling errors, parameter uncertainties and system variations. The most robust configuration is the collocated control, for which as little information as possible about the plant is used in designing the control algorithm, to yield a stable closed-loop system.

When implementing active damping in a motion system, the two control targets, namely motion control and active damping, can be separated. This is called the high authority/low authority control strategy (HAC/LAC), proposed by Seltzer et al. (1988), which is adopted here. This strategy has been implemented among others by Preumont (1997), Verscheure et al. (2006), and Berkhott \& Wesselink (20TT). When the HAC/LAC strategy is applied, two control loops are constructed in parallel; one responsible for motion control (HAC) and the other one for active damping (LAC) (Preumont 1997). Given that the HAC is in general designed for tracking purposes, it has a substantial influence on the system dynamics. This requires wide-band disturbance attenuation and as such, the HAC requires a high gain. This controller is designed on the basis of a model of the structure. Consequently, its stability is sensitive to parameter uncertainties and unmodelled dynamics. LAC has a moderate effect on the plant dynamics and only affects the resonance modes. Thus it typically has a low gain. Although the gain of the LAC is low, it influences the dynamics both within and beyond the bandwidth of the motion controller. The LAC is typically tuned to add optimal 
damping to the modes within the HAC bandwidth, to make the HAC more robust to parameter uncertainties. In this thesis, the LAC, responsible for active damping, is tuned to optimally damp the dominant mode outside the bandwidth of the motion controller, which limits the bandwidth of the motion control loop.

For the active damping loop, piezo actuator and sensor stacks are chosen. Piezo elements have a high accuracy, are small sized, and can be used for a wide range of frequencies. The stacks configuration makes collocation possible.

\subsection{Thesis outline}

The chapters of this thesis are revised versions of journal and conference papers that have either been published, or submitted for publication. As a consequence, the theory used in each chapter is explained in the same chapter. Though this makes repetition unavoidable, it has the advantage of self-contained chapters.

Chapter $\square$ describes the role of damping of vibration modes on the stability of the motion-control loop. Model-based motion controllers are designed using a reduced-order model of the actual plant. The high-frequency dynamics that are not included in the model may influence the stability of the closed-loop system. This chapter presents guidelines concerning the maximum achievable bandwidth for various types of high-order dynamics for $\mathrm{P}(\mathrm{I}) \mathrm{D}$-type motion controllers. Plots of stability regions show the role of damping on the stability of the motion system.

Chapter B gives an overview of the advantages and disadvantages of collocated and noncollocated active damping, as can be found in literature, which mostly address the stability of the active-damping loop and the ease of practical implementation. In addition, this chapter reveals an advantage of collocated active damping for the motion loop, compared to a group of noncollocated methods that use the same actuator as the motion-control loop. This chapter treats the interaction between the active-damping loop and the motion-control loop, with the focus on the performance improvement of the motion control loop.

Chapter 1 combines the theory on the active damping of the rocking mode and the findings of the previous chapters, which is then utilized in a practical implementation for a one-dimensional rotational mode in a motion system. The requirements for achieving the desired active damping are translated into specifications for an active damping unit. These specifications are translated into a mechanical design and a controller that have been incorporated in the test setup.

Chapter $\square$ deals with the effect of modal damping on the three-dimensional dynamics of the end effector. For obtaining the best performance, the active damping unit should be designed to actuate aligned with the vibration axis. However, internal changes of the machine, changes of the physical parameters of the ADU, and the location of the ADU with respect to the compliances in the machine can result in a difference between the modal vibration axis and 
the actuation axis of the ADU. The effect of this on both the active damping performance and the plant dynamics are dealt with in this chapter.

Chapter 1 deals with the stability limitations of the active damping loop due to unavoidable loss of collocation in practice. The results can serve as guidelines when designing a mechanism for active vibration control, as well as during the tuning phase of the vibration controller. 


\section{Chapter 2}

\section{Stability of P(I)D-controlled motion systems}

Abstract - For motion controller design, the reduced plant model is used in which high-frequency dynamics are disregarded. However, the maximum achievable closed-loop bandwidth is limited by the very same dynamics. The extent of their influence depends of the character of the high-frequency modes. Another aspect that has an impact on the stability of the closed-loop system, is the damping present in the plant. The influence of the type of high-frequency dynamics, the damping thereof, and the $\mathrm{P}(\mathrm{I}) \mathrm{D}$ controller bandwidth on the closed-loop stability is addressed in this chapter. The results provide with design rules of thumbs concerning the maximum achievable crossover frequency.

This chapter is a revised version of Babakhani \& de Vries (20106);

"On the Stability properties of P(I)D-controlled motion systems"

B. Babakhani and T. J. A. de Vries

Proceedings of the 5th IFAC Symposium on Mechatronic Systems, Cambridge, MA, USA, Sep. 13-15, 2010. 


\subsection{Introduction}

Model-based motion controllers are designed using a model of the actual plant, based on the knowledge of the system. Some effects within the system, such as static friction and damping, are difficult to model. In addition, a high-accuracy model is time consuming in simulations. Therefore, a reduced model of the actual plant is often used for simulation and control-design purposes. The rules of thumb for tuning motion controllers are similarly based on the reduced models. These low-order models approximate the input-output behavior of the plant as well as possible. However, the high-frequency dynamics that are left out of the model may influence the stability of the system. Therefore, a notion of maximum achievable bandwidth relative to the actual dynamics of the plant, is necessary for the motion control design and/or tuning. The challenge is to determine the highest achievable control bandwidth, given basic information about the lowest disregarded high-frequency dynamics.

A fundamental piece of information needed for the determination of the maximum controller bandwidth, is the resonance frequency, $\omega_{e}$, of the lowest disregarded resonance mode of the plant. Besides $\omega_{e}$, information about any anti-resonance frequency, $\omega_{a}$, is also crucial to determine the stability of the closed-loop system. The order in which $\omega_{e}$ and $\omega_{a}$ appear in the Frequency Response Function (FRF) and their relative distance play an important role in determining the extent of their interference with the stability of the closed-loop system Rankers (1997).

Another important aspect is the damping in the plant. Damping becomes evident in both the phase and amplitude of high-order dynamics and generally contributes greatly to the stability of the system. Yet when designing a plant such as a high-precision industrial machine, damping is often overlooked. This is mostly due to the fact that it is difficult to model and control passive damping in a mechanical structure. In addition, damping is sometimes left out intentionally to improve accuracy and/or conservation of energy. However, damping can also be applied locally within a desired frequency range, by means of active control. The influence of damping on the stability of a closed-loop system justifies the effort put in the implementation of active damping. The scope of this chapter concerns the role of damping in general.

In this chapter, the influence of both high-order dynamics and damping on the stability is investigated for $\mathrm{P}(\mathrm{I}) \mathrm{D}$-controlled motion systems. PID controllers are broadly implemented in industrial applications. According to Äström \& Murray (2001), over $90 \%$ of all control loops are of the PID type. PID controllers and the various tuning methods available are discussed extensively in literature, among others by Ziegler \& Nichols (1942), Skogestad (2004), and Franklin et all (2006). The tuning method used in this thesis is the one presented by van Dijk \& Aarts (2012), which uses the crossover frequency as the key tuning parameter. This 
tunning method has evolved from earlier formulations, such as Coelingh (20(0)), and de Roover (1997).

First, a general plant model in which high-order dynamic characterization is embedded, is introduced in section [2.2. Section 2.3 presents the motion control algorithm along with the tuning rules that lead to the desired closedloop performance. Section 2.4 deals with the influence of both viscous damping present in a plant, and the characteristics of the high-order plant dynamic, on the stability of the closed-loop system, in terms of a maximum achievable bandwidth.

\subsection{Dynamic plant model}

A dynamic model of the plant is assumed to be given in terms of a modal decomposition, which is an intuitive way of representing the dynamics of a system. In this section, a brief overview of modeling in modal coordinates is given. The reader is referred to Meirovitch (1997), Rankers (1997), Harris (1988) for more extensive information and theoretical background of modal analysis.

\section{Modal modeling}

A linear mechanical system without damping can be described by the following general equation of motion:

$$
\boldsymbol{M}_{x} \ddot{\boldsymbol{x}}(t)+\boldsymbol{K}_{x} \boldsymbol{x}(t)=\boldsymbol{F}_{x}(t)
$$

Here, $\boldsymbol{x}$ stands for the generalized displacements, and $\boldsymbol{F}$ for the generalized forces. $\boldsymbol{M}$ and $\boldsymbol{K}$ are respectively the mass and stiffness matrix, which are in general non-diagonal. This set of equations can be decoupled by a transformation on the basis of the solution of the following eigenvalue problem:

$$
\left(\boldsymbol{K}_{x}-\omega_{e, i}^{2} \boldsymbol{M}_{x}\right) \phi_{i}=0
$$

which results in the eigenvalues of the plant, $\omega_{e, 1}, \cdots \omega_{e, n}$, and the corresponding eigenvectors or mode-shape vectors, $\phi_{1}, \cdots, \phi_{n}$. Both the eigenvalues and the direction of the eigenvectors are defined. For the length of the eigenvectors, various scaling methods can be used. The typical choice is scaling the length of each eigenvector such that it is equal to $1,\left|\phi_{i}=1\right|$.

To obtain a set of decoupled equations of motion, a coordinate transformation can be performed using:

$$
\begin{aligned}
\boldsymbol{x}(t) & =\boldsymbol{\Phi} \boldsymbol{q}(t) \\
\boldsymbol{\Phi} & =\left[\phi_{1}, \phi_{2}, \cdots, \phi_{n}\right]
\end{aligned}
$$

This transformation results in the equation of motion in modal coordinates:

$$
\boldsymbol{M}_{m} \ddot{\boldsymbol{q}}+\boldsymbol{K}_{m} \boldsymbol{q}=\boldsymbol{\Phi}^{T} \boldsymbol{F}_{x}
$$


where:

$$
\begin{aligned}
\boldsymbol{M}_{m} & =\boldsymbol{\Phi}^{T} \boldsymbol{M}_{x} \boldsymbol{\Phi} \\
\boldsymbol{K}_{m} & =\boldsymbol{\Phi}^{T} \boldsymbol{K}_{x} \boldsymbol{\Phi}
\end{aligned}
$$

Equation 2.4 has diagonal mass and stiffness matrices, which means that the following orthogonality properties hold:

$$
\begin{aligned}
\phi_{i}^{T} \boldsymbol{M}_{x} \phi_{j} & =0 \quad(i \neq j) \\
\phi_{i}^{T} \boldsymbol{K}_{x} \phi_{j} & =0
\end{aligned}
$$

and

$$
\begin{aligned}
m_{m, i} & >0 \\
k_{m, i} & \geq 0
\end{aligned}
$$

So the equation of motion for the $i$-th eigenmode is:

$$
m_{m, i} \ddot{q}_{i}(t)+k_{m, i} q_{i}(t)=\phi_{i}^{T} \boldsymbol{F}_{x}(t)
$$

Here, $m_{m, i}$ and $k_{m, i}$ respectively represent modal mass and stiffness of the $i$-th mode. The transfer function from the local force $F_{x, j}$ (the $j$-th element of $\mathbf{F}_{x}$ ) to the local position $x_{k}$ (the $k$-th element of $\mathbf{x}$ ) is the sum of each modal contribution, which in turn is determined by the mode-shape vector elements, $\phi_{i j}$ and $\phi_{i k}$ :

$$
\frac{x_{k}}{F_{x, j}}(s)=\sum_{i=1}^{n} \frac{\phi_{i j} \phi_{i k}}{m_{m, i}(s)^{2}+k_{m, i}}
$$

Furthermore, the resonance frequency of the $i$-th vibration mode, $\omega_{e, i}$, is given by:

$$
\omega_{e, i}=\sqrt{\frac{k_{m, i}}{m_{m, i}}}
$$

Since the mechanisms leading to energy dissipation (damping) are complex, it is customary to include damping in the model by incorporating it functionally into the individual modes, instead of performing detailed modeling Karnopp et al. (2006). Including modal damping, $d_{m, i}$, into (2.9) yields the following transfer function:

$$
\frac{x_{k}}{F_{x, j}}(s)=\sum_{i=1}^{n} \frac{\phi_{i j} \phi_{i k}}{m_{m, i} s^{2}+d_{m, i} s+k_{m, i}}
$$

Assuming a viscous model for damping, $d_{m, i}$ can be expressed in terms of the modal damping ratio, $\xi_{i}$, according to:

$$
d_{m, i}=2 \xi_{i} m_{m, i} \omega_{e, i}
$$


Incorporating $([. T 0)$ and $([. T 2)$ in $($ R.TI) results in:

$$
\frac{x_{k}}{F_{x, j}}(s)=\sum_{i=1}^{n}\left(\frac{1}{m_{m, i}} \cdot \frac{\phi_{i j} \phi_{i k}}{s^{2}+2 \xi \omega_{e, i} s+\omega_{e, i}^{2}}\right)
$$

\section{Effective modal parameters}

The value of modal parameters is not unique and depends on the chosen scaling method for the eigenvectors. By transforming the modal parameters into effective modal parameters, unique parameters can be obtained that do not depend on the scaling method and have physical meaning Rankers (1997).

The effective modal parameters of the i-th eigenmode in the physical Degree Of Freedom (DOF) k can be calculated by:

$$
\begin{aligned}
m_{\text {eff }, i, k} & =m_{m, i} / \phi_{i k}^{2} \\
k_{e f f, i, k} & =k_{m, i} / \phi_{i k}^{2} \\
d_{e f f, i, k} & =d_{m, i} / \phi_{i k}^{2}
\end{aligned}
$$

The effective modal parameter in the k-th DOF is basically how a modal parameter is perceived in the k-th DOF of the structure. For instance how a modal stiffness is perceived in the rotational DOF.

The transfer function ([.]1]) in terms of effective modal parameters becomes:

$$
\frac{x_{k}}{F_{x, j}}(s)=\sum_{i=1}^{n}\left(\frac{\phi_{i j}}{\phi_{i k}} \cdot \frac{1}{m_{e f f, i, k} s^{2}+d_{e f f, i, k} s+k_{e f f, i, k}}\right)
$$

\section{Model reduction; fourth-order plant model}

In order to simplify the plant model, model reduction is usually applied to highorder plant models. This results in a model having the rigid body mode and lower-frequency modes that have a significant influence on the dynamic behavior of the plant in the frequency region of interest. In this chapter we consider a fourth order plant having a rigid body mode $(i=0)$ and a flexible mode $(i=1)$, as shown in figure [2.]. Here, $m=0.63 \mathrm{~kg}, \omega_{a}=\omega_{e} / \sqrt{2}$ and $\xi=0.1 \%$. The transfer functions of this plant can be obtained using ([2.TT):

$$
\begin{aligned}
\frac{x_{k}}{F_{x, j}}(s) & =\frac{1}{m_{m, 0} s^{2}}+\frac{\phi_{1 j} \phi_{1 k}}{m_{m, 1} s^{2}+d_{m, 1} s+k_{m, 1}} \\
& =\frac{1}{m_{e f f, 0, k} s^{2}}+\frac{\phi_{1 j}}{\phi_{1 k}} \cdot \frac{1}{m_{e f f, 1, k} s^{2}+d_{e f f, 1, k} s+k_{e f f, 1, k}} \\
& =\frac{(1+\alpha)}{m s^{2}} \cdot \frac{s^{2}+2 \xi_{a} \omega_{a} s+\omega_{a}^{2}}{s^{2}+2 \xi \omega_{e} s+\omega_{e}^{2}}
\end{aligned}
$$




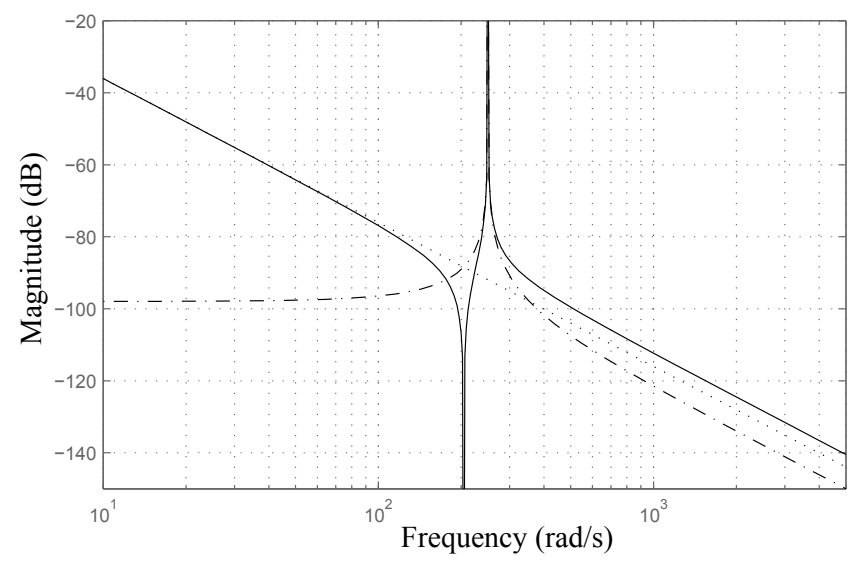

Figure 2.1: Magnitude plot: rigid body mode (dotted), $1^{\text {st }}$ flexible mode at $\omega_{e}=250 \mathrm{rad} / \mathrm{s}$ (dashed) and the resulting $4^{\text {th }}$-order plant transfer function (solide line).

Here:

$$
\begin{aligned}
m & =m_{\text {eff }, 0, k} \\
\alpha & =\frac{\phi_{1 j}}{\phi_{1 k}} \cdot \frac{m_{\text {eff }, 0, k}}{m_{e f f, 1, k}}
\end{aligned}
$$

In general, $\alpha_{i}$ is the factor relating $\omega_{e, i}$ and $\omega_{a, i}$ of the $i$-th eigenmode of a plant according to:

$$
\omega_{a, i}^{2}=\frac{\omega_{e, i}^{2}}{1+\alpha_{i}}
$$

In a mechanical system, $\alpha$ is determined by the location of the sensor w.r.t. the actuator. For $\alpha>0, \omega_{a, i}$ is smaller than $\omega_{e, i}$, implying that in the pole-zero plot there will be a pair of zeros between the rigid-body poles and the poles of the resonance. As $\alpha$ decreases, the zeros in the pole-zero plot move up along the imaginary axis toward infinity where they disappear, to appear again on the real axis when $\alpha$ decreases further (Miu 1992, Preumont 1997). The position of the zeros w.r.t. the resonance poles determines the type of the plant transfer function. Table [2.] shows different ranges of $\alpha$ and the corresponding types of plant transfer functions as discussed by Coelingh (2000), and Soemers \& de Vries $(2008)$. Type $\mathrm{N}$ is not considered hereafter. 
Table 2.1: Plant types.

\begin{tabular}{|c|c|c|}
\hline \hline$\alpha$ & Plant type & Characteristic \\
\hline$\alpha>0$ & Antiresonance - Resonance (AR) & $\omega_{a}<\omega_{e}$ \\
$\alpha=0$ & Unobservable (U) & $\omega_{a}=\omega_{e}$ \\
$-1<\alpha<0$ & Resonance - Antiresonance (RA) & $\omega_{a}>\omega_{e}$ \\
$\alpha=-1$ & Resonance (R) & $\omega_{a}=\inf$ \\
$\alpha<-1$ & Non-minimum phase (N) & zeros at $\pm \omega_{a}$ on the real axis \\
\hline \hline
\end{tabular}

\subsection{Motion controller}

The motion controller is designed to have the minimum bandwidth that is required for the desired performance. The plant is assumed to be a moving mass (high-frequency approximation), the transfer function of which is given by:

$$
P(s)=\frac{1}{m s^{2}}
$$

The controller can be composed of various combinations of proportional, $\mathrm{P}$, integral, I, and differential, D, components. To reduce the high-frequency gain, a low-pass filter can be added to the controller that adds extra high-frequency rolloff. The combination of all the above mentioned components is termed PID+, where the ' + ' sign refers to the additional low-pass filter. The PID+ controller in series form, has the following general transfer function:

$$
\begin{aligned}
C_{P I D+}(s) & =k_{P} \cdot\left(1+\frac{1}{s \tau_{I}}\right) \cdot\left(\frac{s \tau_{D}+1}{\left(s \beta \tau_{D}\right)^{2}+2 \zeta \beta \tau_{D} s+1}\right) \\
& =k_{P} \cdot \frac{\left(s \tau_{D}+1\right)\left(s \tau_{I}+1\right)}{\left(\left(s \beta \tau_{D}\right)^{2}+2 \zeta \beta \tau_{D} s+1\right) s \tau_{I}}
\end{aligned}
$$

where $\beta=0.1 \cdots 0.3$ is the tameness constant of the differentiation action within the motion controller and $\zeta=0.7 \cdots 0.9$ represents the relative damping of the second order roll-off filter. Figure 2.2 shows the Bode plot of a PID+ controller.

$$
\begin{aligned}
k_{P} & =m \omega_{c}^{2} \sqrt{\beta} \\
\tau_{D} & =\left(\omega_{c} \sqrt{\beta}\right)^{-1} \\
\tau_{I} & \geq 2 \tau_{D}
\end{aligned}
$$

Hence, the central parameter by which performance and stability are tuned, is $\omega_{c}$. 


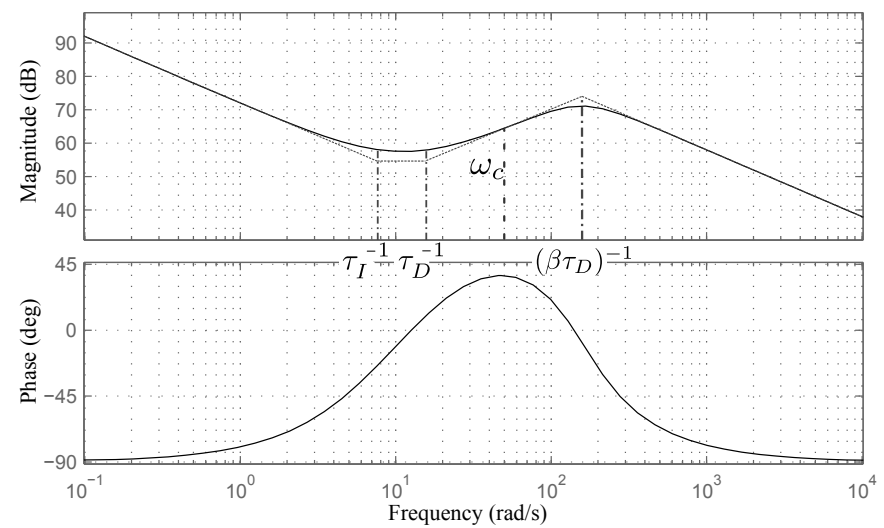

Figure 2.2: Bode plot of PID+ controller; $\tau_{I}=2 \tau_{D}, \beta=0.1, \zeta=0.7, m=0.63 \mathrm{~kg}$ and $\omega_{c}=50 \mathrm{rad} / \mathrm{s}$.

In most cases an encoder mounted on the motor is used for motion-control feedback. So the motion control is collocated. Yet, as a result of both the integral component and the low-pass filter, the motion controller is not passive and thus there is no guarantee for stability, despite the collocation of the actuator and sensor.

\subsection{Stability analysis}

Since the parameter used for tuning the motion controller is the desired $\omega_{c}$, the stability analysis of the system is performed by looking at how it is influenced by variations in $\omega_{c}$.

\subsubsection{Method}

The pole-zero plot of the system is used, where a root-locus (Hahn 1981) is obtained by increasing $\omega_{c}$ from $1^{\text {II }}$ to $\infty$. Note that this is in contrast with the conventional root-locus, where the loop gain is used as the variable to construct the loci with. Figure $\left[2.3\right.$ shows the $\omega_{c}$-based root-locus of the system consisting of the above mentioned plant (see (2.T6) and Figure [2.T) and a PID+ motion

\footnotetext{
${ }^{1}$ Setting the minimum value for $\omega_{c}$ to 0 will result in division by zero in the calculation of $\tau_{D}$. Hence, on the basis of the plant dynamics, an sufficiently low value that is greater than zero should be chosen.
} 


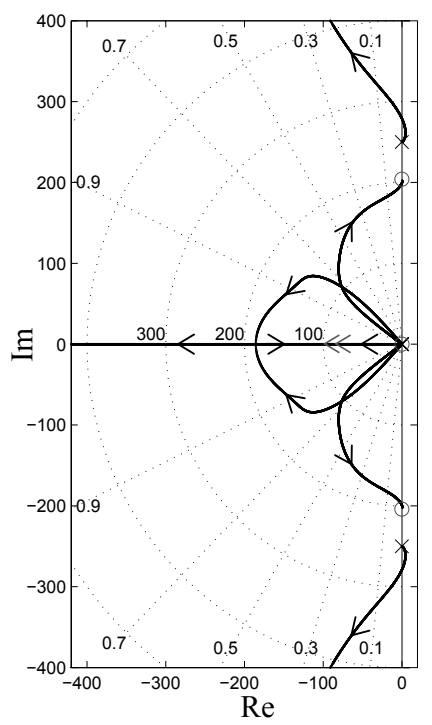

Figure 2.3: Root-locus showing pole-locations as a function of $\omega_{c}$. Note that the two motion-control zeros move along the real negative axis for an increasing $\omega_{c}$, as shown by the two gray arrows.

controller (see (2.201) and Figure [2.2). Using this root-locus plot, a range of $\omega_{c}$ can be deduced, for which the closed-loop system is stable (Borger 2010). It can be seen from figure 2.3 that, due to the resonance poles, this system is unstable for low $\omega_{c}$ and that it becomes and remains stable when $\omega_{c}$ is increased. The latter is only valid under the assumption that no unmodelled high-frequency modes are present.

\subsubsection{Stability; $\omega_{c}$ versus $\alpha$ versus $\xi$}

For several values of damping, $\xi=0.1 \% \cdots 5 \%$, the lower and upper-bound of $\omega_{c}$ have been determined for which a system consisting of a fourth order plant, described by ([.T6), and a PID-type motion controller, as discussed above, is unstable. The results are depicted in Figures 2.4, 2.5), and 2.6, where $\gamma($ (2.22) is plotted versus $\alpha$ (2.18).

$$
\gamma=\frac{\omega_{c}}{\omega_{e}}
$$

The lower bound in each plot is an indication of the maximum achievable bandwidth for which the corresponding closed-loop system is guaranteed to be stable. Uncertainty in plant parameters has not been considered here. This means 
that the stability boundaries can be subject to shift as a result of discrepancies between the model and the plant. Yet the results can still be used as a rule of thumb, when designing motion controllers. Above the upper bound, should one be present, unmodelled modes may exist that can destabilize the system. Hence the stability in this region is uncertain.

\section{PID}

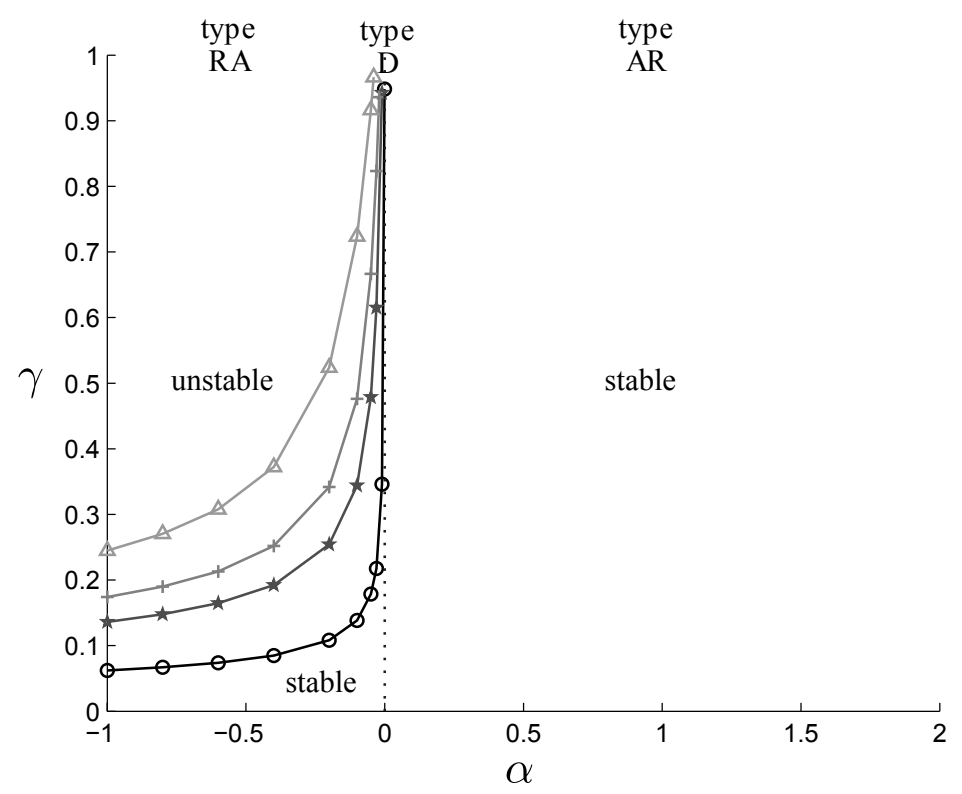

Figure 2.4: Unstable regions; PID without high-frequency roll-off. Increasing $\xi$ from dark to light; $\circ: 0.1 \%, \star: 1 \%,+: 2 \%, \triangle: 5 \%$. Lower bound: solid line, Upper bound: dashed line.

Figure 2.4, shows the stability regions of the PID motion controller ${ }^{\square}$, without high-frequency roll-off, which is described by:

$$
C_{P I D}(s)=k_{P} \cdot\left(1+\frac{1}{s \tau_{I}}\right) \cdot \frac{s \tau_{D}+1}{s \beta \tau_{D}+1}
$$

What strikes most is the poor stability of the RA-type plant transfer in contrast with the stability robustness of the AR type. Damping improves the stability of

\footnotetext{
${ }^{2}$ The stability regions plotted in Figure 2.4 also apply for the PD motion controller without highfrequency roll-off
} 
the RA type considerably, but in case of low damping, it is better to design the control loop such that the plant transfer is of the AR type.

Despite the advantageous stability properties of the AR-type plant transfer in combination with a PID motion controller, it could be undesirable, if not impossible, to implement it in practice. This could for instance be due to the noise present in the system, which in addition to being loud, can wear out the mechanics. More important are the limited dynamics of the actuator and sensor of the motion loop and the required anti-aliasing filter in case of digital control. Thus, having some sort of high-frequency roll-off is inevitable.

PID+

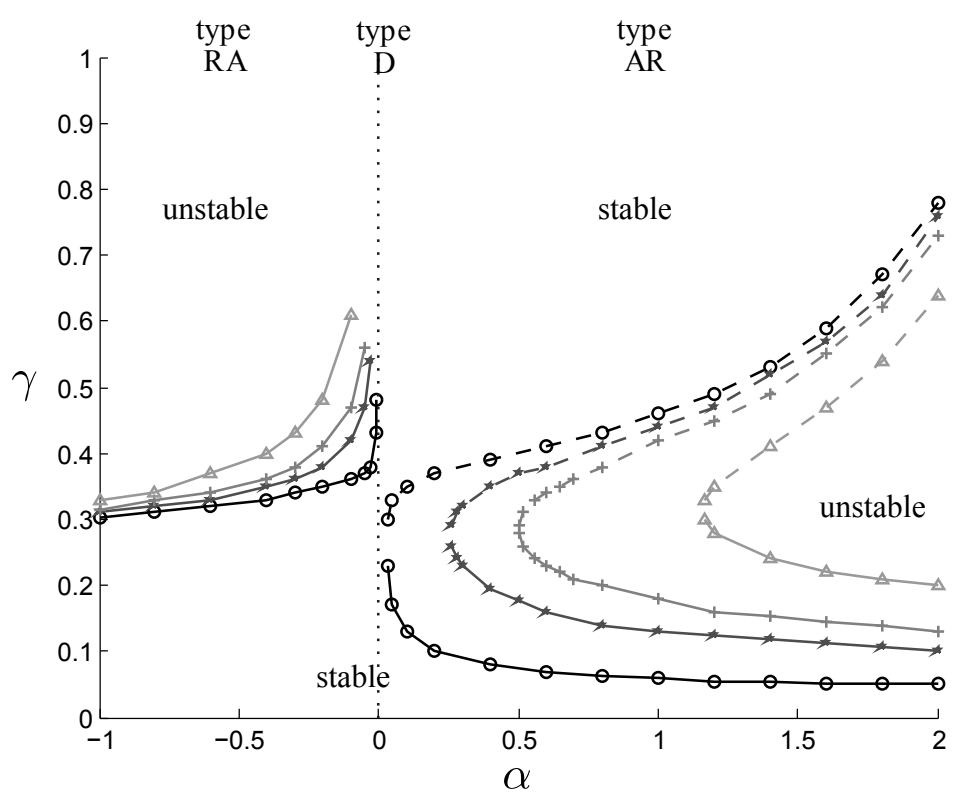

Figure 2.5: Unstable regions; PID+. Increasing $\xi$ from dark to light; $\circ: 0.1 \%$, $\star: 1 \%,+: 2 \%, \triangle: 5 \%$. Lower bound: solid line, Upper bound: dashed line.

The stability regions for the PID+ motion controller (2.201) are shown in Figure 2.5. The first thing that catches the attention is that the lower bound of the RA-type transfer function is larger compared to that of the AR type. Looking at Figure [2.5, $\omega_{c} \leq \omega_{e} / 3$ seems to be an appropriate rule of thumb for obtaining a stable closedloop system for the RA type. As for the AR type, when $\xi=0.1 \%$, which is typically the case for high-precision industrial machines, $\gamma$ should be well below 0.1 . 
For $\alpha=0, \omega_{e}=\omega_{a}$ and the plant transfer functions is equal to that of a moving mass. Since the motion controller is designed for this type of plant, the closed-loop system is stable around this point. The closer $\alpha$ is to zero, i.e. the smaller the distance between $\omega_{e}$ and $\omega_{a}$, the larger the stable $\gamma$ range (higher maximum $\omega_{c}$ ). As damping increases, this region expands simultaneously with the elevation of the lower bound and, if applicable, the decrease of the upper bound of instability region. As $\xi$ increases, the instability vanishes for a growing range of $\alpha$. This effect is more noticeable for the AR-type plant, of which the lower bound of the instability region elevates significantly due to the increase of damping. The relative profit in terms of achievable $\omega_{c}$ is considerable.

PD+

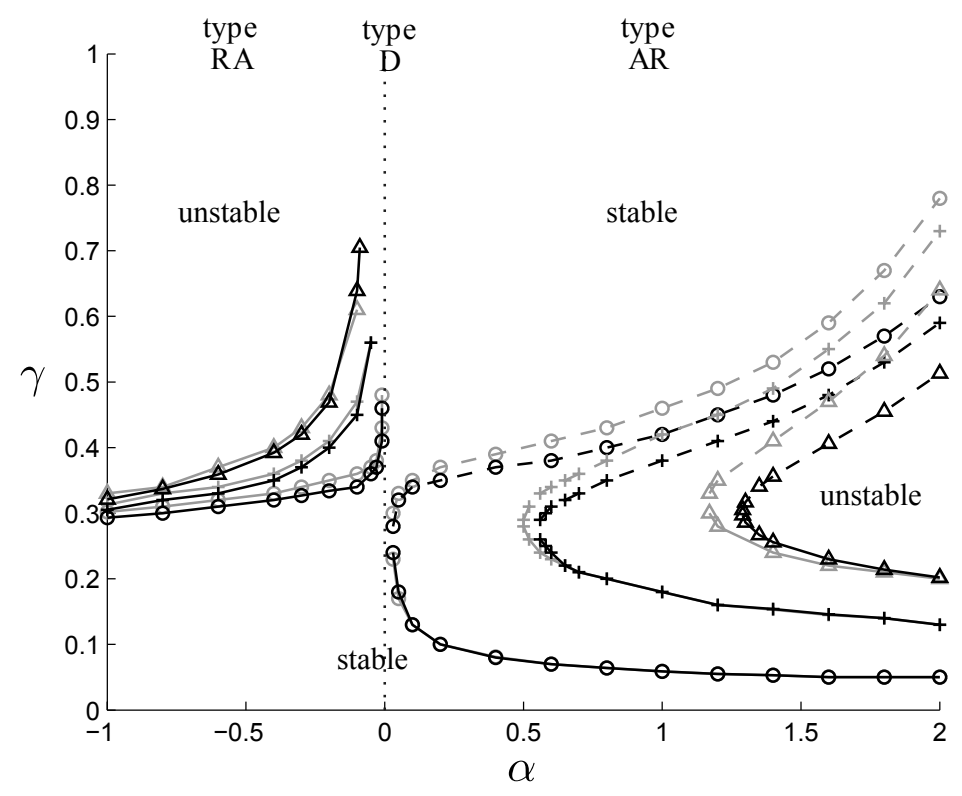

Figure 2.6: Unstable regions; dark: PD+, light: PID+. $\circ: \xi=0.1 \%,+: \xi=2 \%$ and $\triangle: \xi=5 \%$.Lower bound: solid line, Upper bound: dashed line.

Figure 2.6 shows the stability regions for both the PID+ and PD+ motion controller. The transfer function of the PD+ controller is given by :

$$
C_{P D+}(s)=k_{P} \cdot \frac{s \tau_{D}+1}{\left(s \beta \tau_{D}\right)^{2}+2 \zeta \beta \tau_{D} s+1}
$$




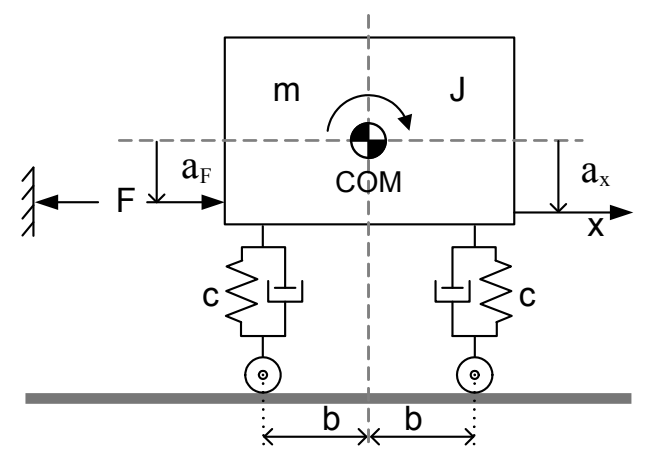

Figure 2.7: Schematic view of the positioning system.

The values considered for $\xi$ are $\xi=0.1 \%, \xi=2 \%$ and $\xi=5 \%$. It can be seen that the unstable region for the RA type of plant transfer function expands over the entire $\alpha$ range. There is a small improvement due to higher damping visible close to the $\alpha=0$ line, but the overall influence of damping is not significant for this type of transfer function.

As opposed to the RA type, the unstable region for the AR-type plants does decrease when no integration is applied in the motion control. This effect becomes more beneficial as $\alpha$ increases. Note that the gain in the stable region is largely due to the shift of the upper bound of the instability region. As mentioned before, the stability in this region may be influenced by the unmodelled high frequency dynamics of the plant and thus, when the knowledge about the plant's high-frequency dynamics is limited, this $\gamma$ range should be avoided. This makes the enlargement of the stable region less useful. However, at the edge of the instability region, for lower values of $\alpha$, also the elevation of the lower bound contributes to the shrinkage of the instability region. The shift of this edge increases as modal damping grows.

\subsection{Application}

Consider a positioning system consisting of a carriage, with $m=0.63 \mathrm{~kg}$, on a linear motor, as depicted in figure 2.]. The guideway of the linear actuator has a certain compliance, which results in vibrations in the transient response of the plant. The compliance is shown by means of two linear stiffnesses, indicated by $c$, in Figure 2.7. We are only interested in the displacement of the carriage in the $x$-direction. The force applied to the carriage, $F$, excites two modes (Rankers 1997):

- rigid body mode, which is a translational movement in the $x$-direction; 
DOF: $x, m_{e f f, 0, x}=m$.

- rocking mode, which is a rotation about the Center Of Mass (COM);

DOF: $\phi, m_{\text {eff }, 1, \phi}=J, k_{\text {eff }, 1, \phi}=2 c b^{2}$.

The resulting displacement of the carriage, $x$, is measured by a position sensor at the distance $a_{x}$ from the COM. The transfer function of the plant (see (2.16)), incorporating both the aforementioned translation and rotation, is given by:

$$
\begin{aligned}
\frac{x_{k}}{F_{x, j}}(s) & =\frac{1}{m s^{2}}+\frac{a_{F} a_{x}}{J s^{2}+2 \xi \sqrt{J k} s+k} \\
& =\frac{1+\alpha}{m s^{2}} \cdot \frac{s^{2}+2 \xi_{a} \omega_{a} s+\omega_{a}^{2}}{s^{2}+2 \xi_{e} \omega_{e} s+\omega_{e}^{2}}
\end{aligned}
$$

Here, $m$ represents the mass of the carriage, $J$ its inertia, $k$ is the rotational stiffness, and $a_{F}$ and $a_{x}$ are the relative positions of the actuator and sensor with respect to the COM. For the damping in the plant, the viscous damping model has been used, resulting in the $2 \xi \omega_{e}$ term in (2.25). Furthermore:

$$
\begin{aligned}
k & =2 c b^{2} \\
\alpha & =\frac{m}{J} a_{F} a_{x} \\
\omega_{e} & =\sqrt{\frac{k}{J}} \\
\omega_{a} & =\sqrt{\frac{k}{J+m a_{F} a_{x}}}
\end{aligned}
$$

The guideway in this example causes a vibration mode at $\omega_{e}=250 \mathrm{rad} / \mathrm{s}$. We assume this mode to be badly damped. Hence, $\xi=0.1 \%$ is used in the plant model, which is a typical value for $\xi$ in high precision machines (Holterman 20(22). The measured displacement of the carriage, $x$, is used as the feedback to the motion controller. The position sensor is mounted such that $\alpha=0.5$. Hence the plant has an AR-type transfer function.

The motion controller is a PID+, with $\beta=0.1$ and $\zeta=0.7$. The position reference signal is a third-degree polynomial with a maximum jerk of $j_{\max }=$ $8 \mathrm{~m} / \mathrm{s}^{3}$. The allowed set-point error is $e_{s p}=1 \mathrm{~mm}$. For this type of motion controller in combination with a third order set-point, the minimum value for $\omega_{c}$, called $\omega_{d}$, which is needed in order to stay within the required set-point error margin, can be determined using:

$$
\omega_{d}^{3}=\frac{2 \cdot j_{\max }}{\beta \cdot e_{s p}}
$$

The aforementioned parameters of this positioning system lead to $\omega_{d}=54 \mathrm{rad} / \mathrm{s}$, 

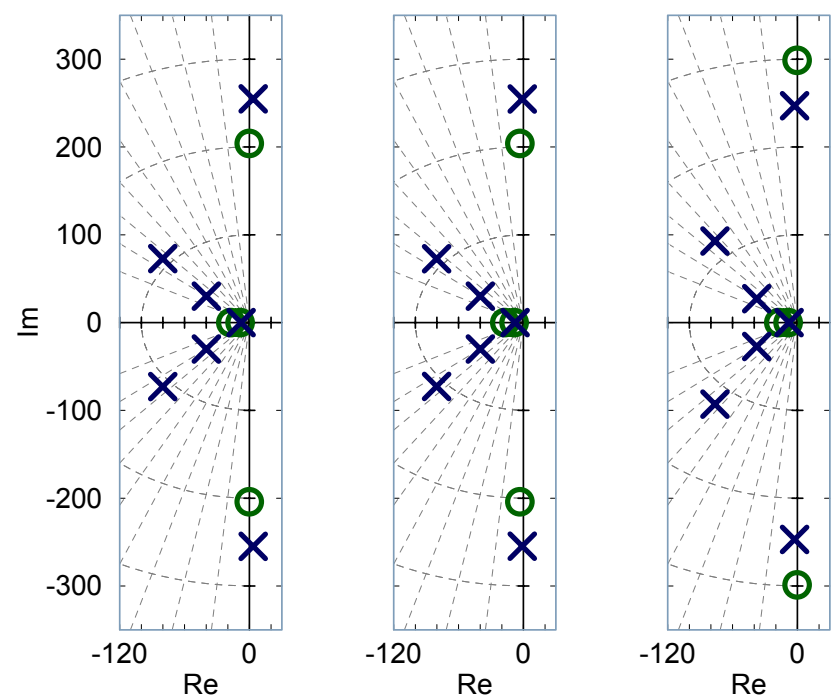

Figure 2.8: Pole-zero plot of the closed-loop system. Left: AR type plant, $\xi=$ $0.1 \%$. Middle: AR type plant, $\xi=2 \%$. Right: $R A$ type plant, $\xi=0.1 \%$.

hence $\gamma=0.22$. Figure 2.5 shows that this value of $\gamma$ is located in the unstable region. The pole-zero plot shown at the left side of Figure 2.8 shows that the complex poles of the plant in the closed-loop system are located in the right-half s-plane.

If possible, the controller should be changed to the PID type, which will yield a stable system according to Figure 2.4. In case this is not possible, using Figure [.5, it can be found that there are two options to stabilize the system. The first option is to move the sensor, in other words change $a_{x}$, such that a pole-zero flipping occurs, as depicted in the right plot in Figure 2.8. The transfer function of the plant will then become of the RA type, which is stable for $\gamma=0.22$. However, it might not be possible to relocate the sensor on the machine in such a way that this change in the transfer function type occurs.

The second option is to increase the damping of the vibration mode. Figure 2.5 shows that $\xi=2 \%$ is high enough to obtain a stable system. This results in the pole-zero plot in the middle in Figure 2.8. If passive means are not sufficient to reach this level of damping, active damping should be considered. Figure 2.9 shows the simulation results with both $\xi=0.1 \%$ and $\xi=2 \%$. The results are as predicted by Figure 2.5. 


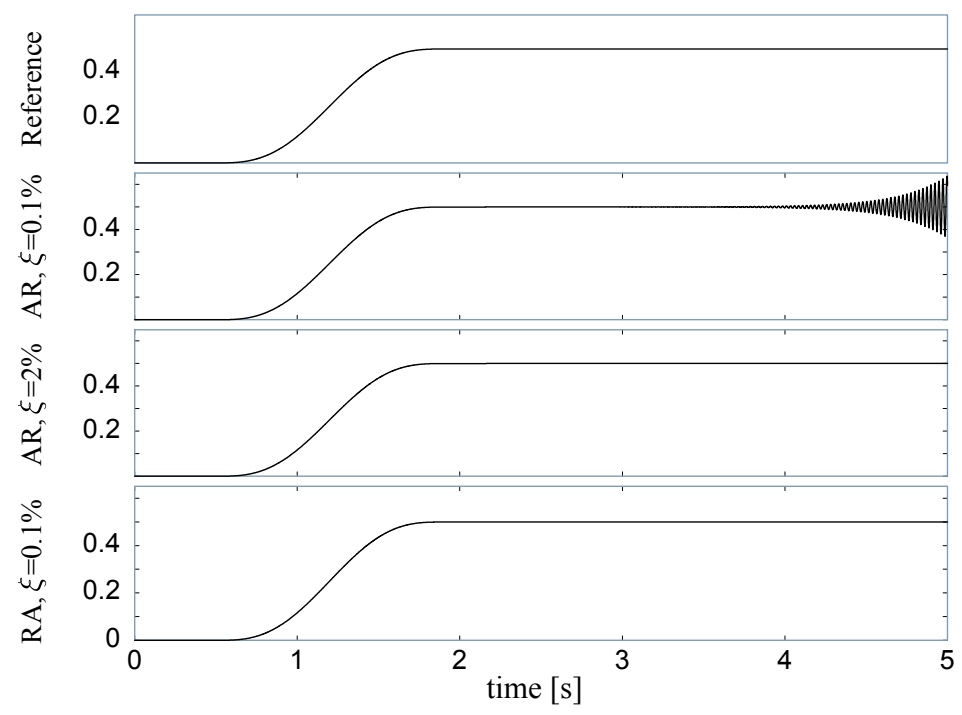

Figure 2.9: Position (m) vs. time (s). Top: third degree polynomial position reference signal. Second: position of the $A R$ type plant, $\xi=0.1 \%$. Third: position of the AR type plant, $\xi=2 \%$. Forth: position of the $R A$ type plant, $\xi=0.1 \%$. 


\subsection{Conclusion}

This chapter analyzed the influence of various types of high-order dynamics on the stability of a closed-loop system, where the controller is tuned using a reduced model of the plant. Results have been summarized in figures [2.4, 2.5. and 2.6. These figures show stable and unstable regions in terms of $\alpha$ (relating antiresonance, $\omega_{a}$, to resonance, $\omega_{e}$ ) and $\gamma$ (relating tuning parameter $\omega_{c}$ to resonance $\omega_{e}$ ) for varying relative damping of the resonance.

The maximum achievable open-loop crossover frequency, $\omega_{c}$, which is directly related to the closed-loop bandwidth, can be deduced from these figures by using no more information than the $\alpha$ and $\xi$ belonging to the most critical high-order dynamics. Hence, the results provide guidelines concerning the achievable $\omega_{c}$, which can be used in the process of motion control design.

The results also show the beneficial effect of structural damping on the stability regions. The extent of the corresponding improvement depends on both the plant type and the type of motion controller. The best performance can be achieved for the AR type of plant transfer function with sufficient damping. 


\title{
Chapter 3
}

\section{Collocated vs Noncollocated Active Damping}

\begin{abstract}
In this chapter, both collocated and noncollocated Active Vibration Control (AVC) of vibrations in a motion system are considered. Pole-zero plots of both the AVC loop and the motion-control loop are used to analyze the effect of the applied active damping on the system dynamics. Using these plots and the simulated end-effector position of the actively damped plant, a comparison is made between the collocated AVC, using Integral Force Feedback (IFF), and noncollocated AVC, by means of acceleration feedback.

It is demonstrated that collocated AVC improves the performance of the plant by adding damping to both the resonance and antiresonance mode of the plant and making it possible to increase the motion-control bandwidth. The applied noncollocated AVC improves the performance by adding damping to the resonance mode. However, as opposed to the collocated AVC, for the applied noncollocated AVC, there is a trade-off between various performance criteria, such as rise time and settling time, that is determined by the balance between the added damping and the increase of the bandwidth. This is true for all the AVC methods that do not increase the damping of the antiresonance mode.
\end{abstract}

This chapter is a revised version of (Babakhani, de Vries \& van Amerongen 2012a);

"A comparison of the performance improvement by collocated and noncollocated active damping in motion systems"

B. Babakhani and T. J. A. de Vries and J. van Amerongen

IEEE/ASME transactions on Mechatronics, to be published. 


\subsection{Introduction}

Resonance is one of the main performance limiting factors in industrial motion systems. For high-performance motion systems, which are designed to have a high mechanical stiffness, little friction and a small load mass, the typical situation is that a PID-type controller is present in the motion-control loop that adds servo stiffness and servo damping to the moving mass dynamics of the plant. The plant will exhibit lightly-damped higher-order vibration modes in addition. These higher-order modes appear as nearly undamped resonances (pole pairs on the imaginary axis) and, depending on the sensor configuration, as nearly undamped antiresonances (zero pairs close to the imaginary axis) in the plant transfer. When the gain of the PID-type Motion Controller (MC) is increased, these resonances will cause vibrations or even instability in the response of the end effector.

An example of such a resonance mode is the so-called rocking mode. The rocking mode phenomenon refers to the rotational vibration mode that is commonly present in motion systems fitted with linear guideways. Due to compliance in the guideway, the cart (end effector) can slightly rotate, i.e. "rock", around its Center Of Mass (COM) when it is driven by an eccentric force, which may result in oscillations in the measured position. This resonance mode restricts the bandwidth of the motion system and its performance.

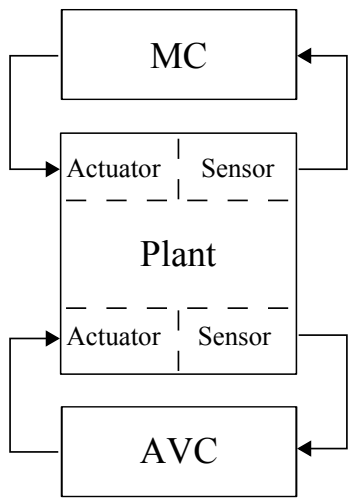

(a) Collocated AVC

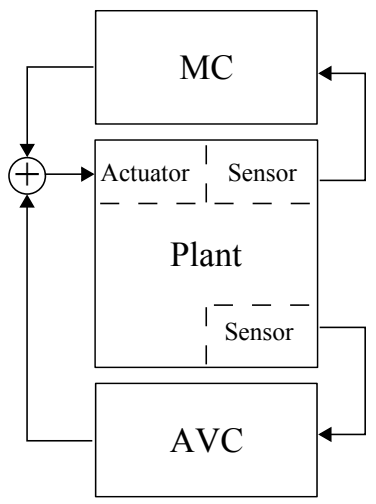

(b) Noncollocated AVC

Figure 3.1: Plant in both MC and AVC closed loop.

By adding damping to the resonance mode of the plant, the oscillations of the end effector can be damped faster, which will result in a shorter settling time. In addition, the previous chapter showed that by adding extra damping to the plant, the maximum achievable bandwidth can be increased. This will reduce 
the response time even further. So, damping improves the performance of the systems on two levels.

Generally, it is quite impossible to realize passive (mechanical) damping in the plant, hence active damping can be considered. And usually, that cannot be realized with the actuator-sensor pair that is in use for motion control, because the observability of the vibration modes in the resulting plant transfer is insufficient. Therefore, it is desirable to add another sensor and possibly another actuator for the purpose of realizing active damping. This leads to two alternative implementations of an Active Vibration Controller (AVC): a noncollocated one, with an additional sensor only, and a collocated one, with an additional actuatorsensor pair (see Figure B.T).

Possible feedback signals are force, position, velocity, or acceleration. The latter three can be converted to one another by means of signal manipulation. Depending on the chosen sensor, various AVC algorithms can be implemented.

When the force is measured, Integral Force Feedback (IFF), which is treated extensively by Preumont (2006) and applied, among others, by Holterman \& de Vries (2004) and Fleming (2010), can be implemented.

Positive position feedback, which was introduced by Goh \& Caughey (1985) and implemented among others by (Denoyer \& Kwak 1996) and Baz \& Poh (1996), is an option for which a position measurement should be performed. For frequency varying structures, the adaptive positive position feedback algorithms can be implemented (Mahmoodi et al, 2010), Hegewald \& Inman 2001, Rew et al. 2002).

When the acceleration of the end effector is known, a second-order filter with sufficient damping can be used to generate a force that is proportional to the acceleration. This force can then be used for active damping (Preumont 1997, Verscheure et al. 2006). The measured acceleration can also be integrated for direct velocity feedback, which is a particular case of lead control (Balas 1979, Yang 1994, Vervoordeldonk et al, 2006).

For the aforementioned active damping methods, the model of the structure is not required. In addition, the stability of the system can be guaranteed by using collocated actuator and sensor pairs, provided that their dynamics are nonrestrictive (Preumont 1997).

The state variable method is a model-based approach that can be applied for AVC, for which the internal states of the system could be estimated using an (implicit) observer. This method is not robust against unmodeled plant dynamics (Fuller 1997).

When applying AVC in a motion system that suffers from the rocking mode, adding damping to the resonance mode of the plant is the means for improving the closed-loop motion performance. The usefulness of collocated AVC for 1D rocking mode has been shown by Babakhani \& de Vries (2010a). This chapter analyzes the effect that active damping in motion systems has on the performance 


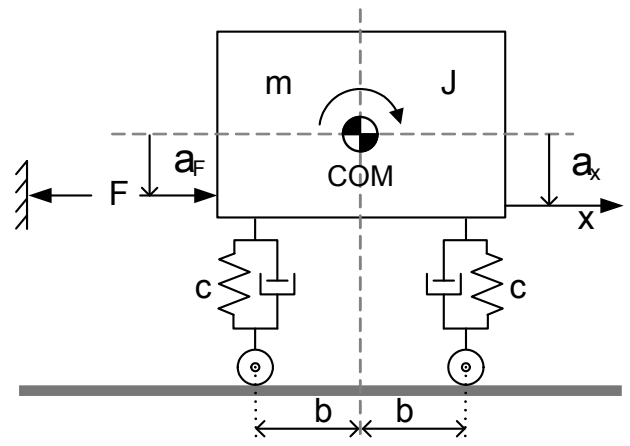

Figure 3.2: $1 D$ model of a plant with rocking mode.

for both the collocated and the noncollocated AVC concept. The emphasis is not so much on choosing the best algorithm for these two control concepts; rather it is on the difference in their effects on the motion-control loop. Assuming that both control concepts are feasible and that the same amount of active damping can be achieved by both, it is interesting to know the resulting performance improvement of the closed-loop system by each concept. The results apply to the class of systems with an internal resonance mode that limits the control bandwidth of the collocated motion controller.

A model of the rocking mode in a one-dimensional setting is presented in Section B.2. The achievable performance in closed loop without AVC is also shown. Section B.3 describes the collocated and the noncollocated AVC and their effect on both the plant and the MC loop. The consequence of including these two vibration controllers for the closed-loop performance is discussed in Section 3.4. The conclusions are presented in Section B.7.

\subsection{Model of the plant}

We are investigating rotational vibration modes in systems with flexible guideways. In a one-dimensional setting, this can be modeled as a fourth-order plant of the flexible guidance class (Coelingh 2000). Figure B.2 shows a schematic representation of this model. It shows a plant, having a mass $m$ and inertia $J$, mounted on a flexible guideway of a linear actuator. The wheels, springs and dampers represent the flexible guideway. The stiffness of the guideway rollers is represented by $c$. This type of constructions typically has a low relative damping (around 0.1\% according to Holterman (20102)), which is represented by the damping icons. $F$ is the actuator force that is to initiate a translational movement $x$. In addition to this translation, $F$ also excites a rocking mode around the $\mathrm{COM}($ rotation $\varphi$ ). This causes a ripple on the measured position of the end 
effector, which limits the performance of the plant.

The transfer function, from the input force $F$ to the end-effector position $x$ of such a plant is given by (see Section 2.2.):

$$
\begin{aligned}
P(s)=\frac{x}{F} & =\overbrace{\frac{1}{m s^{2}}}^{\text {rigid body mode }}+\overbrace{\frac{a_{x} a_{F}}{J s^{2}+R s+k}}^{\text {rotational mode }} \\
& =\frac{1+\frac{m}{J} a_{x} a_{F}}{m s^{2}} \cdot \frac{s^{2}+2 \xi_{a} \omega_{a} s+\omega_{a}^{2}}{s^{2}+2 \xi_{e} \omega_{e} s+\omega_{e}^{2}}
\end{aligned}
$$

where $R$ and $k$ respectively represent the damping and stiffness present in the mechanical structure of the plant, $\xi$ is the damping ratio and $\omega_{e}$ and $\omega_{a}$, respectively, represent the resonance and antiresonance frequency of the plant. $a_{F}$ refers to the actuation arm and $a_{x}$ is the distance between the COM and the displacement location of interest. For the MC loop $a_{x}=a_{x_{M C}}$ and for the endeffector position $a_{x}=a_{x_{\text {perf }}}$.

The relation between the model parameters is as follows:

$$
\begin{aligned}
R & =2 \xi_{e} J \omega_{e} \\
& =2 \xi_{a}\left(J+m a_{F} a_{x}\right) \omega_{a} \\
k & =2 c b^{2} \\
\omega_{a} & =\sqrt{\frac{k}{J+m a_{F} a_{x}}} \\
\omega_{e} & =\sqrt{\frac{k}{J}}
\end{aligned}
$$

For motion control, a PID controller with high-frequency roll-off is applied. Such a controller is generally implemented in commercial off-the-shelf servo drives, and hence has wide applicability. On the basis of the desired performance the minimum required crossover frequency, $\omega_{c}$, is determined. This $\omega_{c}$ is used for tuning the motion controller (see Section $[2.3$ ).

Figure 3.3 shows how the poles of the closed-loop system move in the complex plane for an increasing crossover frequency $\omega_{c}$, where $m=0.65 \mathrm{~kg}, k=50$ $\mathrm{Nm} / \mathrm{rad}, \xi_{e}=2 \%, a_{F}=0.12 \mathrm{~m}, a_{x_{M C}}=0.12 \mathrm{~m}$ and $\omega_{e}=73 \mathrm{rad} / \mathrm{s}$. Note that the two MC zeros move along the negative real axis for an increasing $\omega_{c}$ (the two gray arrows in Figure (3.3). From this plot it becomes clear that $\omega_{c}=13 \mathrm{rad} / \mathrm{s}$ is the maximum achievable crossover frequency for the system having the above mentioned parameters. It seems that the system stabilizes for high $\omega_{c}$ 's. However, this can only happen when there is no higher-order mode in the vicinity of the modeled vibration mode. Otherwise, the higher-order mode(s) can influence the locus of the modeled resonance mode in such a way that it remains in the right half-plane. Also it is possible that the unmodeled higher-order modes destabilize 


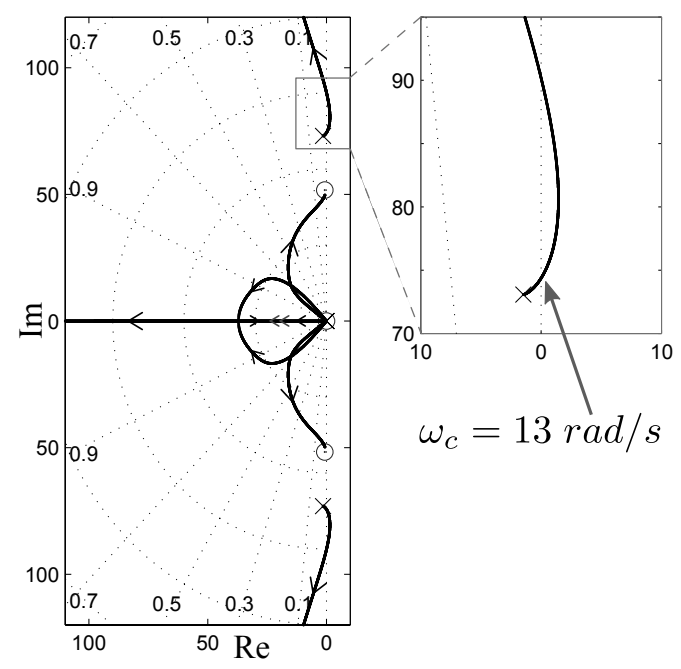

Figure 3.3: Loci of the poles and zeros of the plant with low damping in motioncontrol loop for a varying $\omega_{c}$.

the system for a high $\omega_{c}$. Therefore, the $\omega_{c}$ for which the modeled resonance mode destabilizes the system should be considered the maximum achievable $\omega_{c}$.

\subsection{Active damping}

The adopted control structure for adding active damping in this chapter is the well known high authority/low authority control (HAC/LAC), shown in Figure 3.4, where the LAC is responsible for active damping and the HAC is dedicated to the motion control purpose (Preumont 1997, Seltzer et al, 1988). These two controllers operate in parallel, but independent of each other. So all in all, there are three transfer functions that play a role in the dynamics of the closed-loop system:

- $H_{\text {perf }}$, where $L_{\text {perf }}=P_{\text {perf }} \cdot C_{M C}$

- $H_{M C}$, where $L_{M C}=P_{M C} \cdot C_{M C}$

- $H_{A V C}$, where $L_{A V C}=P_{A V C} \cdot C_{A V C}$

In general, $H_{\text {perf }}$ is equal to $H_{M C}$, except that the plant zeros are not present. The system is described by: 


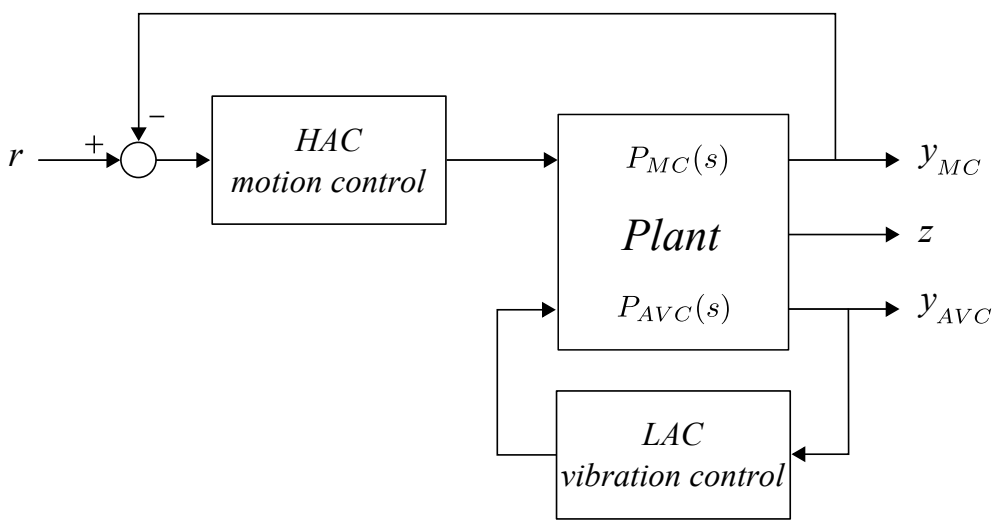

Figure 3.4: The control scheme including both the motion controller and the active vibration controller according to the HAC/LAC strategy.

$$
\left[\begin{array}{c}
z \\
y_{M C} \\
y_{A V C}
\end{array}\right]=\left[\begin{array}{cc}
H_{\text {perf }} & 0 \\
H_{M C} & 0 \\
0 & H_{A V C}
\end{array}\right]\left[\begin{array}{c}
r_{M C} \\
r_{A V C}
\end{array}\right]
$$

Where $z$ is the end-effector state of interest (in this case its position), $y_{M C}$ represents the output of the sensor for $\mathrm{MC}, y_{A V C}$ is the state of the plant used for vibration control and $r_{M C}$ and $r_{A V C}$ are the references for the MC and AVC, respectively.

There are two control concepts, namely the collocated control concept and the noncollocated control concept, referring to the locations of the controller actuator and sensor with respect to each other. The motion controller is assumed to be collocated. For the active damping, either one can be implemented on the basis of the situation at hand. In the following subsections, both alternatives are discussed. Preumont (1997) gives a full description of all the control algorithms that are used in this chapter.

\subsubsection{Collocated AVC}

Collocated control means that the sensor and actuator in the control loop are energetically conjugated, which leads to the following properties:

+ Guarantee of stability: a collocated sensor and actuator pair forms a power port between the controller and the plant. This makes it possible to control the power flow to the plant. As a consequence, by applying an energetically passive or a dissipative controller -in this case the AVC-, the stability of the 


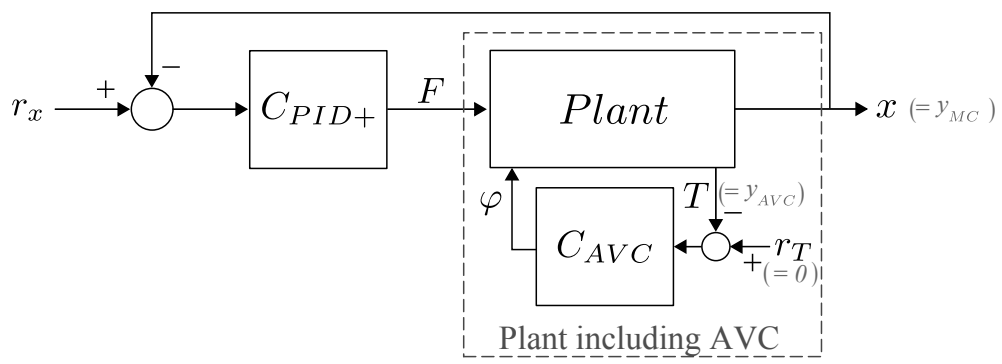

Figure 3.5: The control scheme including both the motion controller and the collocated active vibration controller.

plant-when the active vibration controller is included-is guaranteed ${ }^{\text {W. }}$.

+ Robustness: collocated control is robust against possible system parameter variations and unmodeled dynamics (Preumont 1997).

- Practical limitations: the mechanical structure of a plant can make it challenging, or even impossible, to put the sensor and actuator at the same location.

The control scheme including the collocated AVC is shown in Figure 3.5. Active damping can be applied by measuring any torque present and counteracting it using a position actuator $\left(r_{A V C}=r_{T}=0\right)$. By placing the actuator and sensor at the same location, for instance by using a piezo actuator and sensor stack, collocated control can be realized. By dividing the plant into a carrier and the end effector as shown in Figure B.6, the so-called Active Damping Unit (ADU) -consisting of the actuator and sensor stack for the AVC - can be placed between the guideway and the end effector. In Figure B.6, the wheels, the translational stiffnesses and dampers of Figure B.2, representing the guideway, are replaced by their rotational counterparts, $k$ and $R$. The mass of the carrier is assumed to be negligibly small and is therefore not included in the models. The ADU consists of a torque-sensor and a rotation actuator that is shown in parallel with a stiffness, $k_{p l}$. This stiffness is used to apply the necessary preload force over the piezo actuator in the ADU.

\section{AVC algorithm}

Although the sensor and actuator of the aforementioned ADU are collocated, the measured and actuated variables (torque, $T$, and rotation $\phi$, respectively) are not dual. Since duality is a necessary condition for guaranteeing stability,

\footnotetext{
${ }^{1}$ This of course only holds if there are no limitations imposed by the electronics, actuators and sensors used.
} 


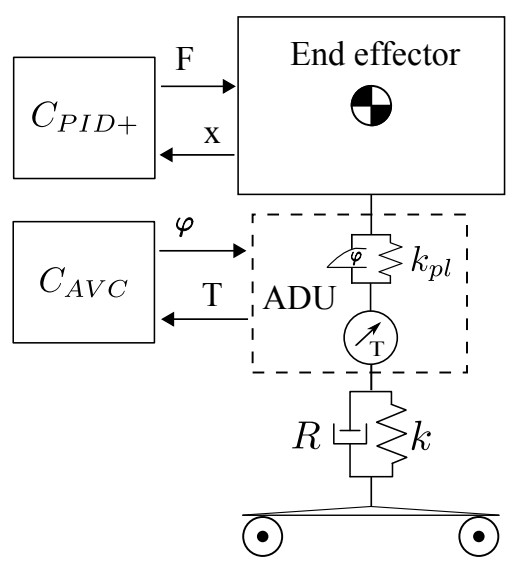

Figure 3.6: Schematic view of the plant in combination with both collocated AVC and motion controller.

it is desirable that an energetically intrinsic passive relation between the corresponding dual variables, namely torque and velocity, is realized. This can be achieved by choosing an appropriate control algorithm, such as IFF (Preumont 1997, Holterman \& de Vries 20(05), which basically results in (viscous) damper dynamics between the dual variables $T$ and $s \phi$ :

$$
\begin{aligned}
C_{I F F}(s) & =\frac{K_{I F F}}{s} \\
\phi(s) & =\frac{K_{I F F}}{s}\left(r_{T}(s)-T(s)\right) \\
& =-\frac{K_{I F F}}{s} T(s) \\
K_{I F F}^{-1} s \phi(s) & =-T(s) \\
d_{A V C} s \phi(s) & =-T(s)
\end{aligned}
$$

Equation (B.5) shows that the power flow from the controller to the plant, $P_{C \rightarrow P \text {, }}$ is always non-positive.

$$
\begin{aligned}
d_{A V C} s \phi(s) & =-T(s) \\
d_{A V C} \dot{\phi}(t) & =-T(t) \\
P_{C \rightarrow P}(t) & =\dot{\phi}(t) T(t) \\
P_{C \rightarrow P}(t) & =-d_{A V C} \dot{\phi}^{2} \leq 0
\end{aligned}
$$

To avoid actuator saturation, a forgetting factor is added to $C_{I F F}(s)$. The resulting algorithm is termed Leaking Integral Force Feedback (LIFF) (3.6). This 


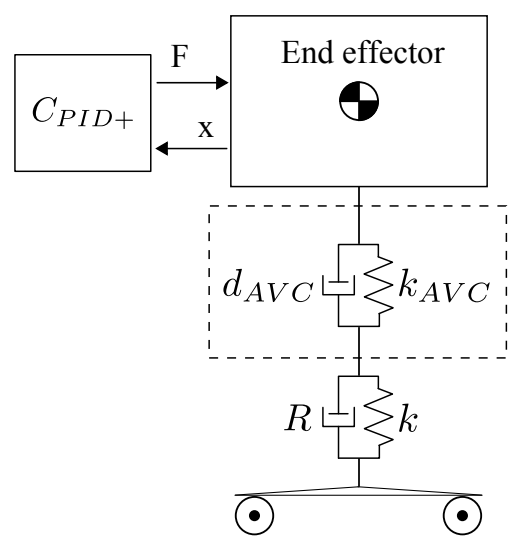

Figure 3.7: Schematic view of the plant in combination with both collocated $A V C$ and motion controller. The collocated AVC is represented by its dynamic behavior (compare with Figure 3.6 ).

control algorithm is in fact a $1^{\text {st }}$ order low-pass filter, having the gain $K_{L I F F}$. The forgetting factor results in spring dynamics between $T$ and $s \phi$, as can be seen from (B.6).

$$
\begin{aligned}
C_{L I F F}(s) & =\frac{K_{L I F F}}{s+p_{L P}} \\
K_{I F F}^{-1} s \phi(s)+p_{L P} K_{I F F}^{-1} \phi(s) & =-T(s) \\
d_{A V C} s \phi(s)+k_{A V C} \phi(s) & =-T(s)
\end{aligned}
$$

So by choosing the LIFF as the AVC algorithm, the following holds:

$$
\begin{aligned}
C_{A V C}(s) & =\frac{K_{L I F F}}{s+p_{L P}}=\frac{1}{d_{A V C} s+k_{A V C}} \\
d_{A V C} & =K_{L I F F}^{-1} \\
k_{A V C} & =p_{L P} K_{L I F F}^{-1}
\end{aligned}
$$

Hence, this controller behaves as a mechanical damper $d_{A V C}$, for high-frequencies (higher than $p_{L P}$ ), and as a stiffness $k_{A V C}$, for low frequencies (see Figure B.7, and so it is energetically passive ).

The parameters of the collocated AVC have been tuned according to the rules of thumb deduced by Holterman (2002). This tuning method merely uses the target $\omega_{e}$ of the plant. Consequently, by using this method any model uncertainty is irrelevant.

The transfer function of the plant in the AVC loop, from the ADU actuator to its 
sensor, is:

$$
P_{A V C}(s)=\frac{T}{\varphi}=k_{p r} \cdot \frac{J s^{2}+R s+k_{s r}}{J s^{2}+R s+k}
$$

Where:

$$
\begin{aligned}
k_{p r} & =k_{p l}+k \\
k_{s r}^{-1} & =k_{p l}^{-1}+k^{-1}
\end{aligned}
$$

\section{Filter}

Adding any filter to this controller will result in the loss of passivity and therefore the guarantee of stability is lost as well. Still, for improving the static stiffness of the plant when the AVC is included, a high-pass filter, described by (B.TU), might be added to the AVC. If the cut-off frequency of this filter is small w.r.t. the resonance frequency, the closed-loop stability is preserved (Holterman 2002).

$$
H_{H P}(s)=\frac{s}{s+p_{H P}}
$$

The transfer function of the plant in the motion-control loop, when the active vibration controller is included becomes:

$$
P_{M C}(s)=\frac{x}{F}=\frac{1}{m s^{2}} \cdot \frac{\left(J+a_{x} a_{F} m\right) s^{2}+(R+\psi) s+\kappa k}{J s^{2}+(R+\psi) s+\kappa k}
$$

Where:

$$
\begin{aligned}
\psi & =\frac{k}{s+p_{L P}+H_{H P}(s) K_{L I F F}\left(k_{p l}+k\right)} \\
\kappa & =\frac{p_{L P}+H_{H P}(s) K_{L I F F} k_{p l}}{s+p_{L P}+H_{H P}(s) K_{L I F F}\left(k_{p l}+k\right)}
\end{aligned}
$$

Note that both the poles and the zeros of the MC loop are damped by the collocated AVC.

\section{Realizable active damping}

Considering the transfer function of an AVC loop, the distance between its poles and zeros in the pole-zero plot is the measure for the controllability of that mode. The poles represent the resonance frequency of the plant $\omega_{e}$, and the zeros of the AVC loop $\omega_{a_{A V C}}$, the resonance frequency of the subsystem constrained by the ADU actuator and sensor (Miu 1992). The larger the distance between the two, the higher the controllability of a certain mode, and therefore, the larger the 


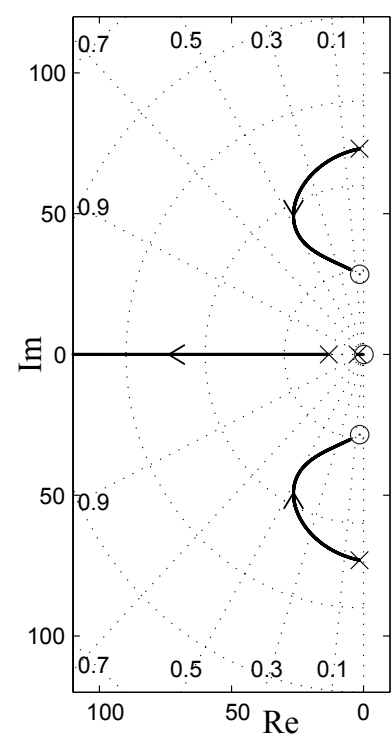

(a) root-locus of $P_{A V C}(s)$ (B.8), $\varphi \rightarrow T$

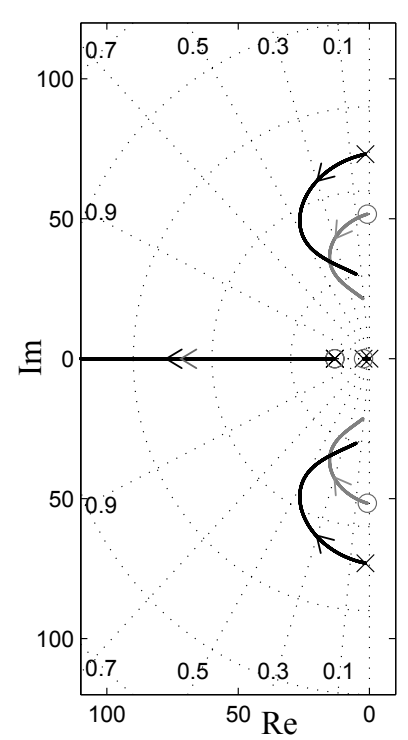

(b) loci of the poles and zeros of $P_{M C}(s)$ (B.TI), $F \rightarrow x$

Figure 3.8: Loci of the poles (black line) and zeros (gray line) of the plant including collocated $A V C$, with high-pass filter (3.T0), for a varying $K_{L I F F}$.

maximum achievable damping. The maximum achievable damping for $\omega_{a_{A V C}}>$ $\omega_{e} / 3$, can be determined according to (Preumont 1997):

$$
\xi_{e}^{\text {max }}=\frac{\omega_{e}-\omega_{a_{A V C}}}{2 \omega_{a_{A V C}}}
$$

Effect on $P_{A V C}$ and $P_{M C}$

Equation (B.TI) shows that the AVC can add damping to both poles and zeros of the MC loop, as mentioned previously. This can also be seen in Figure 3.8 which shows the loci of the poles and zeros of the plant including the collocated AVC, for a varying $K_{L I F F}$ (where $k_{p l}=9 \mathrm{Nm} / \mathrm{rad}, p_{L P}=0.18 \cdot \omega_{e}$ and $p_{H P}=0.17 \cdot p_{L P}$ ). Figure 3.8 a shows that the resonance poles travel to the zeros of the AVC loop when $K_{L I F F}$ is increased. $\xi_{e}$ increases at first, but after achieving its maximum value, it decreases when $K_{L I F F}$ is increased further. The same trend applies for the $\omega_{a_{M C}}$. Figure $\overline{3.8 \mathrm{~b}}$ shows that $\xi_{a_{M C}}$ changes simultaneously and to the same extent as $\xi_{e}$ for an increasing $K_{L I F F}$.

Besides the increase of $\xi_{e}$ for the target resonance mode, this active damping algorithm also increases $\xi_{e}$ of the higher modes of the plant that were not 


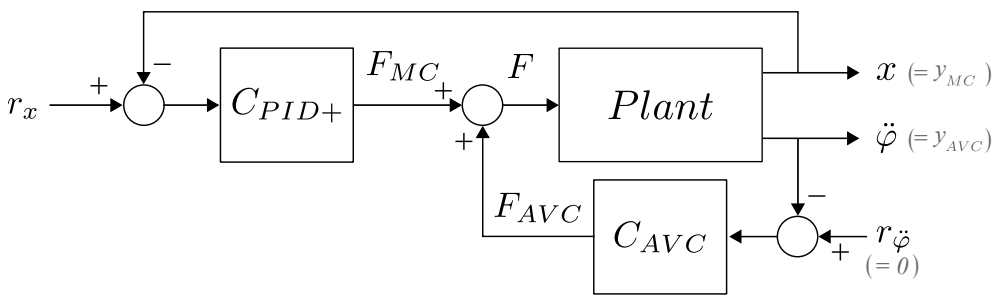

Figure 3.9: The control scheme including both the motion controller and the noncollocated active vibration controller.

included in the reduced model (B.]). The extent of the resulting $\xi_{e}$ for the higher modes is generally unknown because of the lack of information about these modes. So the higher-order modes can put an upper bound on the achievable $\omega_{c}$.

\subsubsection{Noncollocated AVC}

When noncollocated control is applied, sensing and actuating take place at different parts of the plant. This has the following consequences:

+ Practical implementation: the advantage of noncollocated AVC is that it is relatively easy to implement in the plant. After all, just adding a sensor to the plant takes less effort than adding both a sensor and an actuator.

- No guarantee of stability: unlike for collocated control, noncollocated control can jeopardize the stability of the system due to its effect on the residual modes.

- Less robust: noncollocated control is less robust against parameter variations and discrepancies between the used plant model and the actual plant.

To obtain a better observability of the performance limiting vibration mode, a sensor can be added to the plant. Here, the noncollocated AVC uses the acceleration of the end effector that can be measured by means of an acceleration sensor; $r_{A V C}=r_{\ddot{\varphi}}=0$. It is relatively easy to measure the absolute acceleration of the end effector compared to, e.g., its absolute position and/or velocity. The AVC uses the same actuator as the MC. Figure B.9 shows a schematic overview of the control-loops acting on the plant.

\section{AVC algorithm}

Equation (B.13) shows the transfer function of the AVC, where $g$ represents the gain. Due to the integral action in the controller, this is in effect the same concept 
as the direct velocity feedback (Preumont 1997).

$$
C_{A V C}(s)=\frac{g}{s}
$$

The transfer function of active damping control loop is:

$$
P_{A V C}(s)=\frac{\ddot{\varphi}}{F_{A V C}}=\frac{a_{F} s^{2}}{J s^{2}+R s+k}
$$

\section{Filter}

To decrease the interference of the AVC with the MC and to suppress any effect of high-frequency modes and/or noise, both a high-pass and a low-pass filter, described by (3.15), have been added to the AVC.

$$
H_{H L}(s)=\frac{1}{s+p_{L P}} \cdot \frac{s}{s+p_{H P}}
$$

The integral action on the acceleration is canceled by the high-pass filter. So any drift that might have been caused by the integration is hereby excluded. As a whole, the AVC including the filters is in effect the same as feeding the measured acceleration to a second order filter, $H(s)$, in combination with a proportional controller (Preumont 1997), represented by the following equation:

$$
H(s)=\frac{g}{s^{2}+2 \xi_{f} \omega_{f} s+\omega_{f}^{2}}
$$

where:

$$
\begin{aligned}
\omega_{f} & =\sqrt{p_{L P} \cdot p_{H P}} \\
\xi_{f} & =\frac{p_{L P}+p_{H P}}{2 \sqrt{p_{L P} \cdot p_{H P}}}
\end{aligned}
$$

The center frequency of this filter, $\omega_{f}$, is chosen equal to $\omega_{e}$.

The transfer function of the plant when the AVC including the band-pass filter is included becomes:

$$
P_{M C}(s)=\frac{1}{m s^{2}} \cdot \frac{\left(J+a_{x} a_{F} m\right) s^{2}+k}{J s^{2}+\left(R+H_{H L}(s) g a_{F}\right) s+k}
$$

Effect on $P_{A V C}$ and $P_{M C}$

Given that $g$ only appears in the denominator of (B.18), it is clear that the noncollocated AVC only adds damping to $\omega_{e}$. Figure B.10 shows the loci of the poles and zeros of the plant including the noncollocated AVC for a varying $g$ 


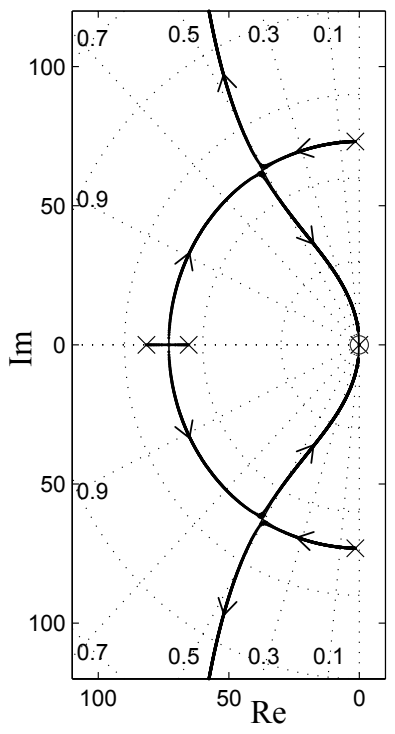

(a) root-locus of $P_{A V C}(s)$ (B.T4), $F_{A V C} \rightarrow \ddot{\varphi}$

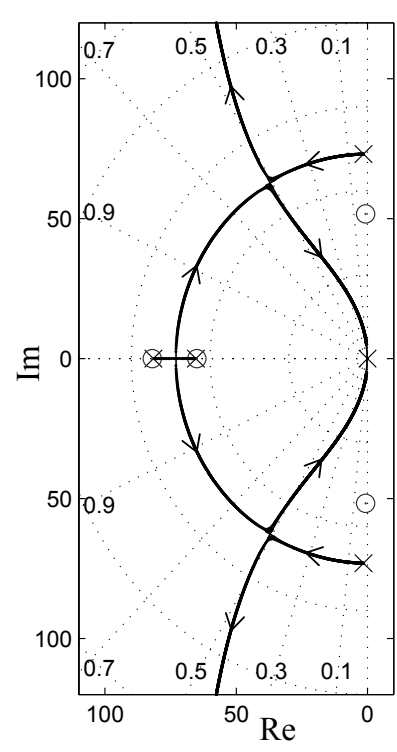

(b) loci of the poles and zeros of $P_{M C}(s)$ (B.T8), $F_{M C} \rightarrow x$

Figure 3.10: Loci of the poles and zeros of the plant including noncollocated AVC for a varying $g, \omega_{f}=\omega_{e}$.

(where $p_{L P}=82$ and $p_{H P}=65$ ). It shows that increasing $g$ increases $\xi_{e}$ to a certain extent, beyond which $\xi_{e}$ decreases as the poles move towards the zeros of the AVC loop, located at the origin as depicted in B.10a. The amount of damping added to the resonance mode depends on $g, a_{F}$ and ratio of $\omega_{f}$ to $\omega_{e}$. Note that $\xi_{a_{M C}}$, shown in B.10b, is unaffected by the noncollocated AVC. Therefore, there is no gray line representing the movement of the zeros, unlike the collocated AVC case that was shown in Figure 3.86.

\subsection{Results; AVC in motion control closed loop}

Since active damping has been applied in a motion system, the total system including both the AVC and the MC should be considered when it comes to evaluating the performance.

Figure $3 . T]$ shows a part of the locus of the $H_{M C}$ poles for $\omega_{c}$ varying from 1 to $\infty$. The loci of both the resonance and the rigid-body poles for the plant including the collocated AVC is shown in B.11a. Figure B.110 shows the same for the noncollocated AVC. 


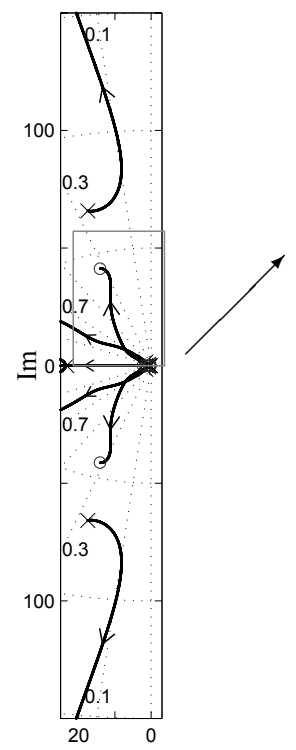

(a) collocated
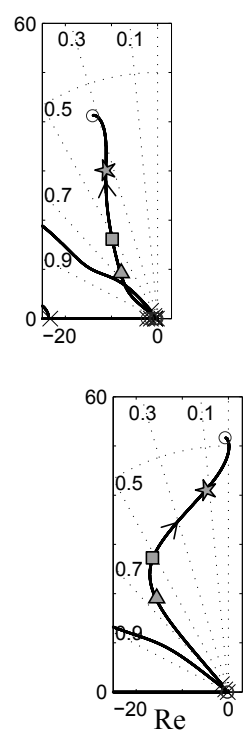

(b) zoomed in

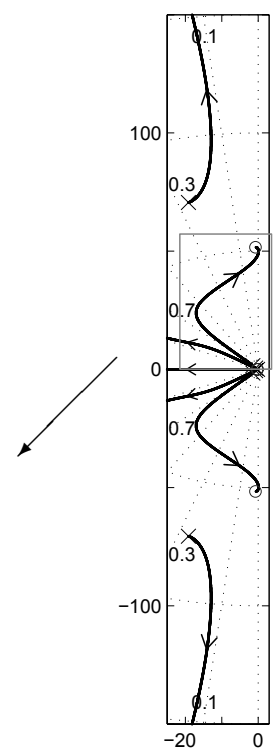

(c) noncollocated

Figure 3.11: Locus of the poles of the motion-control loop including AVC for a varying $\omega_{c} ; \mathbf{\Delta} \omega_{c}=13 \mathrm{rad} / \mathrm{s}, \mathbf{\square}: \omega_{c}=20 \mathrm{rad} / \mathrm{s}, \star: \omega_{c}=50 \mathrm{rad} / \mathrm{s}$.

These figures show that for an increasing $\omega_{c}, \xi_{e}$, mostly achieved by the AVC, decreases for both the collocated and noncollocated AVC. In other words, the poles shift toward the imaginary axis, yet stay stable and damped.

More important is the effect of an increasing $\omega_{c}$ on the poles in the origin. These rigid-body poles move toward the zeros of the MC loop. The noncollocated AVC does not add damping to these zeros so they are located very close to the imaginary axis. So the relative damping of the closed-loop poles that move toward these zeros when $\omega_{c}$ is increased (in this case the rigid-body poles), decreases. This counteracts any improvement of the performance achieved by the added active damping to the resonance mode. The reader is referred to Section 3.6 for more information on the balance between active damping and $\omega_{c}$.

Moreover, since the MC operates in a collocated fashion, the MC loop zeros are at a lower frequency than the resonance mode. This means that the oscillations due to the poles in the vicinity of these zeros will have a higher amplitude than the oscillations caused by the resonance mode. Thus, although the oscillations due to the resonance mode have been damped by the noncollocated AVC, the rigid-body poles cause oscillations in the plant's response. 
Table 3.1: Performance of the plant with and without AVC $\left(\xi_{e}=0.25\right)$, in closed loop.

\begin{tabular}{|c|c|c|c|c|}
\hline \hline AVC & $\begin{array}{c}\omega_{c} \\
(\mathrm{rad} / \mathrm{s})\end{array}$ & $\begin{array}{c}\text { Overshoot } \\
(\%)\end{array}$ & $\begin{array}{c}\text { Rise-time } \\
(\mathrm{s})\end{array}$ & $\begin{array}{c}\text { Settling time } \\
(s), \text { error }<1 \%\end{array}$ \\
\hline No & 13 & $53 \%$ & 0.12 & $>>2$ \\
\hline \multirow{3}{*}{ Collocated } & 13 & $22 \%$ & 0.13 & 0.70 \\
& 20 & $17 \%$ & 0.09 & 0.47 \\
& 50 & $32 \%$ & 0.05 & 0.19 \\
\hline \multirow{3}{*}{ Noncollocated } & 13 & $37 \%$ & 0.12 & 0.62 \\
& 20 & $44 \%$ & 0.08 & 0.41 \\
& 50 & $75 \%$ & 0.05 & 0.54 \\
\hline \hline
\end{tabular}

The decrease of performance for high motion-control bandwidths was also mentioned in (Verscheure et al, 20016), where an $H_{\infty}$ AVC and a lead-lag MC implemented, without any explanation however.

This is a major difference between the noncollocated method for AVC and the collocated method. In the latter case the MC loop zeros are damped to the same extent as the poles. Since the rigid-body poles remain in the vicinity of the $\mathrm{MC}$ loop zeros, the decrease of their relative damping for an increasing $\omega_{c}$ is limited and will not become significantly smaller than the damping added by the collocated AVC.

It should be noted that the zeros of $H_{M C}$ do not appear in the performance loop, $H_{\text {perf }}$ (see Section 3.3). This means that the distance between the closed-loop poles and the $H_{M C}$ zeros does not play a role in the closed-loop performance. It is the location of the closed-loop poles in the complex plane that should be considered for performance.

\subsection{Simulated step responses}

Figure B.T2 shows the simulated step response of the plant with and without active damping, where both the collocated AVC and the noncollocated AVC are tuned to yield $\xi_{e}=0.25$. The model parameters are the same as in Section 3.2. Furthermore, $a_{x_{M C}}=0.12 \mathrm{~m}$ and $a_{x_{\text {perf }}}=-0.08 \mathrm{~m}$. These plots together with Table 3.1 confirm the conclusions drawn previously from the pole-zero plots. The simulations also show a significant improvement in terms of overshoot for collocated AVC. Both collocated and noncollocated AVC reduce the settling time considerably. However, there is a trade-off between decreasing the rise- 


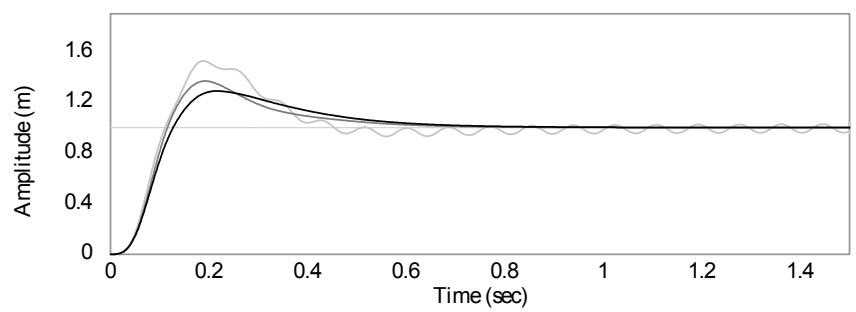

(a) $\omega_{c}=13 \mathrm{rad} / \mathrm{s}$

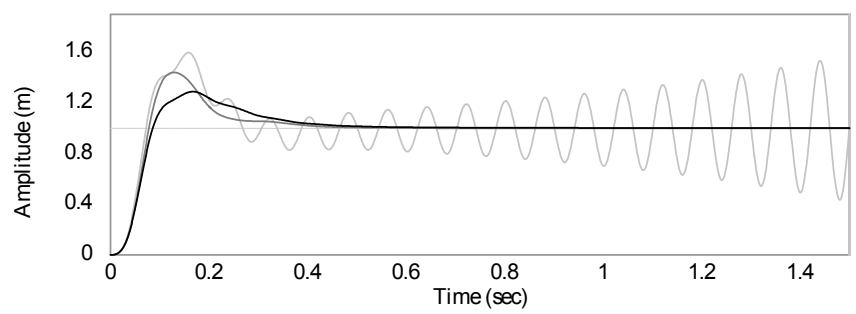

(b) $\omega_{c}=20 \mathrm{rad} / \mathrm{s}$

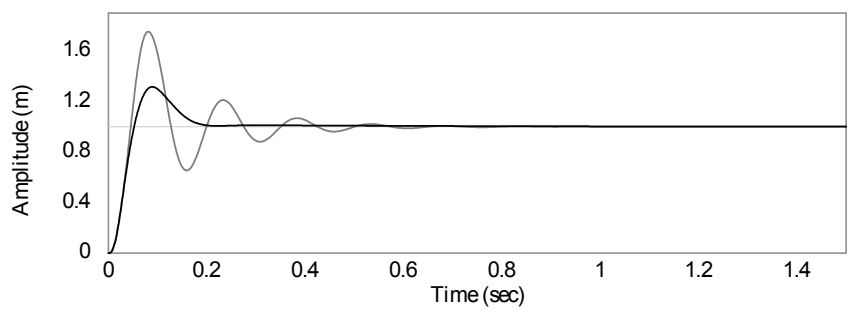

(c) $\omega_{c}=50 \mathrm{rad} / \mathrm{s}$

Figure 3.12: Step responses of the closed-loop system for various $\omega_{c}$ 's, amplitude versus time; light gray: No AVC, dark gray: noncollocated $A V C$ with $g=300$, black: collocated AVC with $K_{L I F F}=0.7$. 
time (increasing $\omega_{c}$ ) and decreasing the settling time (increasing the relative damping of the poles), beyond a certain $\omega_{c}$ for the noncollocated AVC control. The noncollocated AVC can of course be optimized and the control algorithm itself can be altered to achieve better damping characteristics. Nevertheless, the fact remains that as long as the $\mathrm{MC}$ loop zeros have a low relative damping, the system performance does not benefit from active damping for high $\mathrm{MC}$ bandwidths.

\subsection{Balance between active damping and $\omega_{c}$}

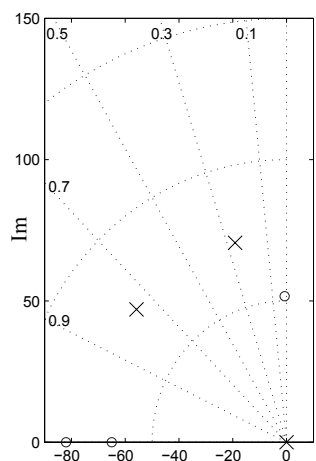

(a) No MC, $g=300$

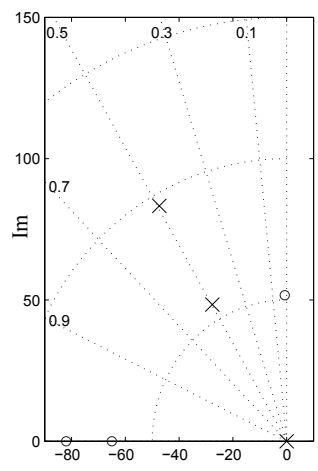

(d) No MC, $g=500$

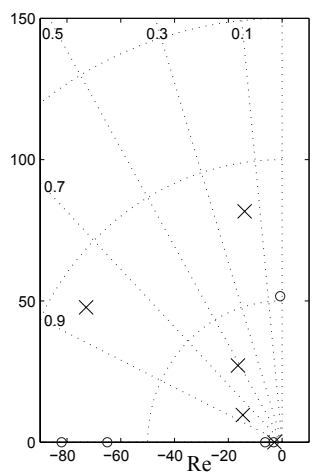

(b) $\omega_{c}=20, g=300$

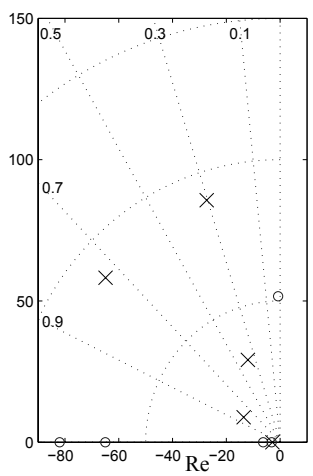

(e) $\omega_{c}=20, g=500$

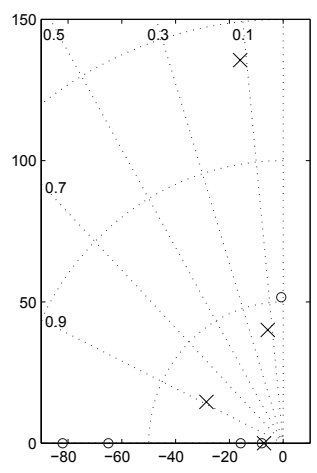

(c) $\omega_{c}=50, g=300$

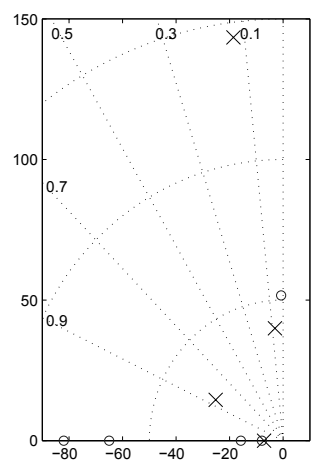

(f) $\omega_{c}=50, g=500$

Figure 3.13: A part of the pole-zero plot of the plant including the noncollocated $A V C$, without MC ( $a$ and $d)$ and in motion control closed loop (b, $c$, e and $f$ ), for various values of $g$ and $\omega_{c}(\mathrm{rad} / \mathrm{s})$. 
The closed-loop poles for various values of $\omega_{c}$ and $g$ are shown in Figure B.T3. The two values of $g$ in this figure are $g=300$ that results in $\xi_{e}=0.25$ and $g=500$ that results in $\xi_{e}=0.5$, which is the highest achievable damping for the noncollocated AVC, as shown previously in Figure B.TU. For convenience only a small part of the pole-zero plot is shown, which contains the most relevant information concerning the performance. Figure B.T3 shows that when $\xi_{e}$ is increased, the rigid-body poles move faster toward the undamped closedloop zeros for an increasing $\omega_{c}$. The higher the relative damping of the resonance poles, the faster the rigid-body poles approach the imaginary axis when increasing the MC bandwidth. So the combination of maximum achievable damping and bandwidth does not necessarily yield the best performance. The latter is confirmed by the step responses depicted in Figure 3.14, where $a_{x_{M C}}=$ $0.12 \mathrm{~m}$ and $a_{x_{\text {perf }}}=-0.08 \mathrm{~m}$. It shows that for a high $\omega_{c}$, a lower noncollocated active damping is more beneficial. The balance between the applied active damping and $\omega_{c}$ for the best performance should be determined using the plant model. So the achieved performance improvement depends on the available plant knowledge.

For comparison, also the step responses of the closed-loop system including the collocated AVC, having the same $\xi_{e}$ 's and $\omega_{c}$ 's, are shown in Figure 3.15 . These step responses show that the performance of the closed-loop system, including the collocated AVC, does not vary significantly by variations in either the AVC gain or the $\omega_{c}$.

\subsection{Conclusion}

In motion systems, AVC can be used to add damping to vibration modes (poles) and therewith allows for bandwidth increase. This chapter shows that an important difference between collocated and noncollocated active damping is that only in the collocated case, damping is added to the antiresonances (zeros) of a vibration mode. As a result, for the noncollocated case, a further bandwidth increase eventually leads to closed-loop poles in the vicinity of the imaginary axis and consequently oscillations in the closed-loop dynamics worsening the performance. Hence, noncollocated active damping only improves the performance for low MC bandwidths. In contrast, for the collocated case the closed-loop poles remain well damped and additional performance gain is obtained. 


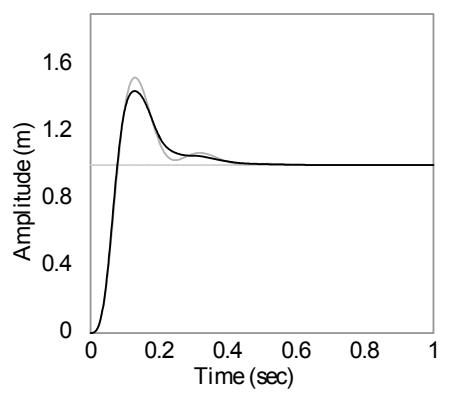

(a) $\omega_{c}=20$

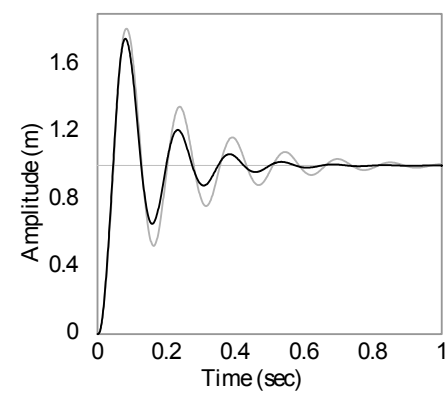

(b) $\omega_{c}=50$

Figure 3.14: Step responces of the closed-loop system including the noncollocated $A V C$, for various values of $\omega_{c}$ and $g$; gray: $g=500$ and $\xi_{e}=0.5$, black $g=300$ and $\xi_{e}=0.25$.

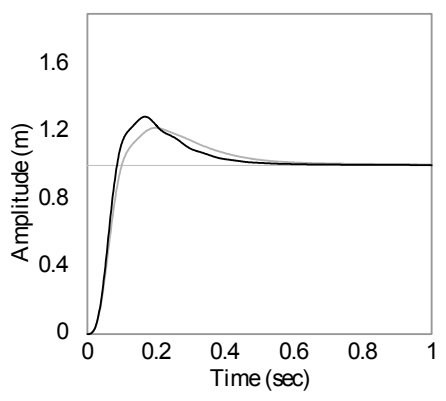

(a) $\omega_{c}=20$

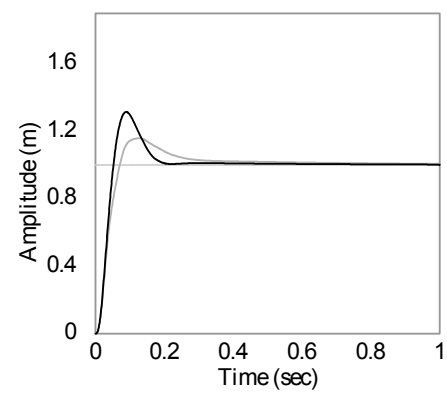

(b) $\omega_{c}=50$

Figure 3.15: Step responces of the closed-loop system including the collocated $A V C$, for various values of $\omega_{c}$ and $K_{L I F F}$; gray: $K_{L I F F}=1.5$ and $\xi_{e}=0.5$, black: $K_{L I F F}=0.7$ and $\xi_{e}=0.25$. 
50 CHAPTER 3. COLLOCATED VS NONCOLLOCATED ACTIVE DAMPING 


\title{
Chapter 4
}

\section{Experimental Evaluation of Active Damping}

\begin{abstract}
A rotational vibration mode is a performance limiting phenomenon that typically occurs in machines fitted with linear actuation. Active damping of this mode in motion systems allows for increasing the motion-control bandwidth and results in a better performance. This chapter describes an experiment designed to show the effect of active damping. After deriving a set of requirements from the dynamical model, the mechanical design of an active damping unit containing collocated actuator and sensor pairs is described. The implemented active vibration controller algorithm is a leaking integral force feedback, which makes use of force measurement and position actuation. Measurements show a five times higher relative damping of the resonance mode after implementing active damping. The improvement in the transient response of the test setup is evident in the measured end-effector position.
\end{abstract}

This chapter is a revised version of (Babakhani, Brouwer, de Vries \& van Amerongen 2012);

"Experimental Evaluation of Active Damping of a Rotational Vibration Mode in Motion Systems"

B. Babakhani, and D. M. Brouwer, and T. J. A. de Vries and J. van Amerongen submitted to IEEE/ASME Transactions on Mechatronics. 


\subsection{Introduction}

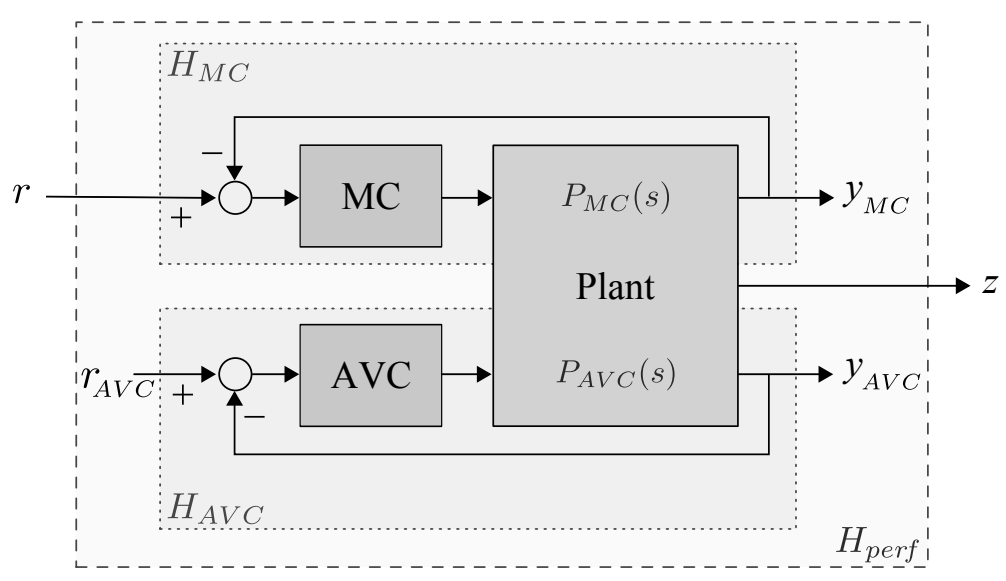

Figure 4.1: Control scheme for both the motion control (MC) and the active vibration control (AVC) loop. $z$ : the end-effector state of interest, $r$ : the reference for $z, r_{A V C}$ : the reference for the $A V C$, which is equal to zero, $H_{M C}$ : the transfer function of the MC loop from $r$ to $y_{M C}, H_{A V C}$ : the transfer function of the AVC loop from $r_{A V C}$ to $y_{A V C}, H_{\text {perf }}$ : the transfer function of performance loop from $r$ to $z$.

Resonance in industrial machines is an important performance liming factor. An example of such a vibration is the rotational vibration that is present in plants fitted with linear actuation. The compliance of the guideway in such a plant induces a poorly damped rotational vibration in the end effector. This rotational vibration mode is known as the rocking mode, referring to the 'rocking' motion of the end effector around the guideway.

These vibrations are usually not (sufficiently) observable and/or controllable in the motion control transfer $H_{M C}$ (see Figure 4.T). Thus the motion controller (MC) cannot inject (enough) damping into these modes, which results in performance degradation. Adding damping will improve the performance by reducing the settling time of the end effector. In addition to its effect on the performance loop $H_{\text {perf }}$, damping also allows for a higher bandwidth of the motion controller as shown in Chapter $\rrbracket$, which in turn improves the system performance in terms of both settling time and low-frequency disturbance rejection. When damping by passive means is proven insufficient or not realizable with the applicable requirements such as maximum weight and cost, active damping can provide a solution.

Active damping can be implemented in both a collocated and a noncollocated manner (Preumont 1997, Holterman 20172, Anderson \& Hagood 1994, van 
Schothorst 1999). Collocated damping has been chosen in this chapter because of its stability robustness and its superior performance improvement properties compared to the noncollocated active damping (see Chapter B). The active damping controller (AVC) operates in parallel with, but independently of the motion controller. This control structure is the high authority/low authority control, HAC/LAC, proposed by (Seltzer et al. 1988) and implemented among others by Preumont (1997), Symens et al. (2003), and Pereira et al. (2011). The resulting control scheme is depicted in Figure 4.1 .

This concept of collocated active damping of the rocking mode is presented in Chapter 3 and (Babakhani \& de Vries 2010a), where the effect of active damping on the plant performance has been shown using simulations. In this chapter, the results of previous chapters are combined in an implementation of active damping on a test setup. This chapter deals with practicalities of AVC and presents measurement results showing the effect of damping on the performance of a motion system.

First, a model of the kind of plant considered here is given in Section 4.2. Also, the test setup made to show the effect of the rocking mode and the damping thereof, on the dynamics of a plant, is introduced in this section. Section 4.3 describes both the theory behind the selected active damping algorithm, and its practical implementation in the test setup. The measurement results are discussed in Section 4.4, leading to the conclusions presented in Section 4.5.

\subsection{Plant}

In this section a dynamic model of a plant suffering from the rocking mode is presented in terms of a modal decomposition. The test setup used for demonstrating the effect of rocking mode and the implementation of active damping is introduced along with the expected achievable performance on the basis of plant parameters.

\subsubsection{Plant model}

Considering vibrations to be small motions around an equilibrium allows to use linear equations of motion (L.T) to describe their dynamics. Using this equation, a general plant transfer function in terms of modal coordinates can be obtained by summation of all modal contributions, as described in Section 2.2. So, considering a lightly-damped motion system suffering from the rocking mode, a reducedorder model incorporating both the plant's rigid body mode $(i=0)$ and its (most dominant) rocking mode $(\mathrm{i}=1)$ is given by (4.T). This equation describes the transfer function from the $j$-th force input $F_{x, j}$, to the $k$-th position output $x_{k}$ 
(see also (2.25)):

$$
\begin{aligned}
\frac{x_{k}}{F_{x, j}}(s) & =\frac{1}{m s^{2}}+\frac{a_{F} a_{x}}{J s^{2}+R s+k} \\
& =\frac{1+\alpha}{m s^{2}} \cdot \frac{s^{2}+2 \xi_{a} \omega_{a} s+\omega_{a}^{2}}{s^{2}+2 \xi_{e} \omega_{e} s+\omega_{e}^{2}}
\end{aligned}
$$

where, assuming a viscous model for damping:

$$
\begin{aligned}
\alpha & =\frac{m}{J} a_{F} a_{x} \\
R & =2 \xi_{e} \sqrt{J k} \\
\xi_{a} & =\xi_{e} / \sqrt{1+\alpha}
\end{aligned}
$$

In case $x_{k}$ is the end-effector state of interest, (4.T) represents the plant transfer function for the performance loop, $P_{\text {perf }}$. The plant transfer function belonging to the motion-control loop, $P_{M C}$, is in general of the same form, where $x_{k}$ stands for the state that is used for feedback to the motion controller. The feedback loop usually makes use of a position measurement performed by an encoder mounted on the motor (collocated control).

\subsubsection{Rocking mode demonstrator}

The Rocking Mode Demonstrator (RMD), shown in Figure 4.2, is a setup designed and realized by Imotec b.v. (one of the industrial SmartPie project partners) to visualize both the effect of the rocking mode, and damping thereof, on the dynamics of a plant (Borger \& Holterman 2010). To this end, the following requirements should be satisfied:

1. Mimicking the rocking mode.

Realization: for demonstration purposes, the vibrations are exaggerated by implementing a local compliance, in the form of a leaf spring, in the setup, mimicking the rocking mode. By doing so, a new, isolated, vibration mode is introduced in the low-frequency range, which is more dominant than the modes caused by other compliances in the setup ${ }^{m}$.

2. Facility for measuring both the MC feedback and the performance.

Realization: this setup is fitted with two sensors; an encoder mounted on the motor carrier, which enables collocated position measurement $\left(x_{e n c}\right)$ for motion control, and a magnetic position sensor for noncollocated position measurements $\left(x_{x s e n s}\right)$ that can be used as an indication for performance at the end-effector location. The position sensor can be shifted underneath the bar, and hence positioned w.r.t. the Center Of Mass (COM) of the bar.

\footnotetext{
${ }^{1}$ Hence, among others the compliance in the guideway of the RMD can be disregarded.
} 


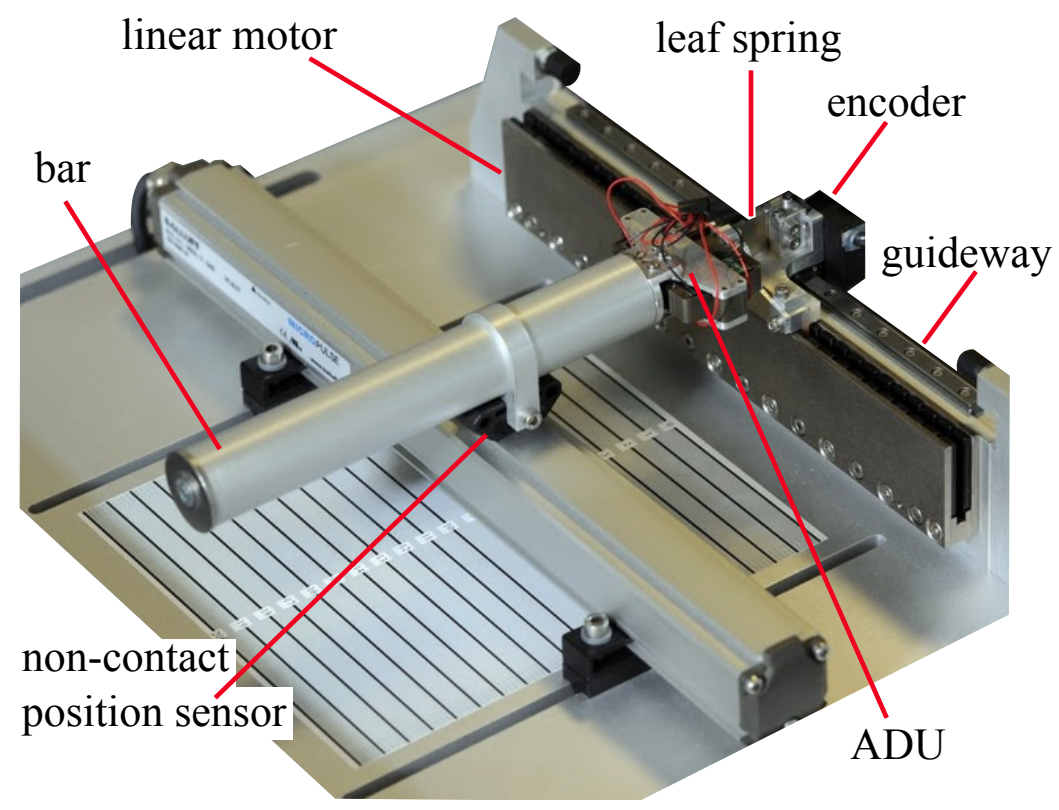

Figure 4.2: Picture of the RMD, side view.

3. Low friction.

As mentioned before, the leaf spring causes a low-frequency resonance mode. This means that the maximum achievable MC bandwidth is low. As a consequence, it is important to have a low friction.

4. Low damping.

Precision machines suffer from low-damping modes; in general $\xi_{e} \leq 0.01$. To simulate the effect of the rocking mode realistically, the RMD should be designed with low damping.

Realization: the RMD is designed to have low-weight moving parts and high-stiffness constructions.

Figure 4.31 shows a schematic picture of the RMD, where the relevant parameters are highlighted (see Table 4.7 for the parameter values). The actuation of the motor carrier is assumed to be aligned with its COM. 
a.
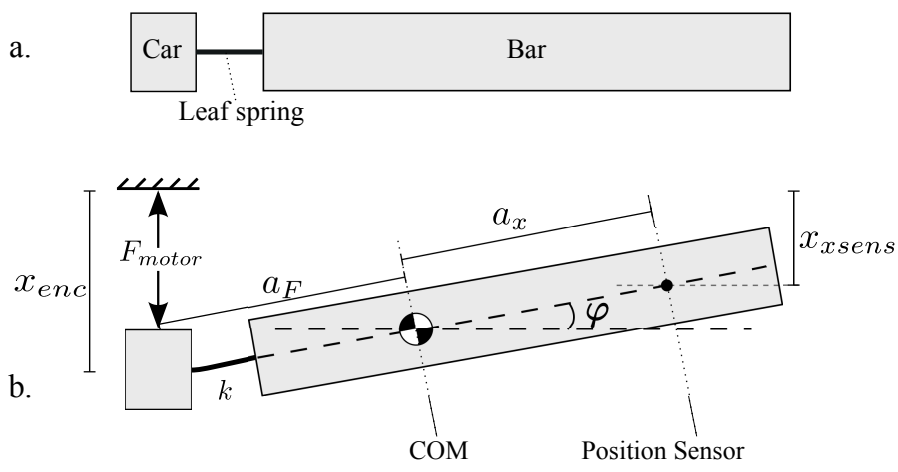

Figure 4.3: Schematic top view of the RMD (a) and its relevant parameters (b); $x_{\text {enc }}$ : collocated position measurement by encoder, $x_{x s e n s}$ : noncollocated position measurement by position sensor.

\subsubsection{Motion control loop}

For motion control the following PID control algorithm with a high-frequency roll-off has been chosen (see Chapter [1):

$$
C_{P I D+}(s)=k_{P} \cdot \frac{\left(s \tau_{D}+1\right)\left(s \tau_{I}+1\right)}{\left(\left(s \beta \tau_{D}\right)^{2}+2 \zeta \beta \tau_{D} s+1\right) s \tau_{I}}
$$

When tuning this control algorithm, the plant is assumed to be a moving mass. The rigid-body mass of the plant and the desired crossover frequency are used to tune the controller. For the RMD, $m=0.6 \mathrm{~kg}, \zeta=0.7, \beta=0.1$, and the following relations hold:

$$
\begin{aligned}
k_{P} & =m \omega_{c}^{2} \sqrt{\beta} \\
\tau_{D} & =\left(\omega_{c} \sqrt{\beta}\right)^{-1} \\
\tau_{I} & =2 \tau_{D}
\end{aligned}
$$

Table 4.1: RMD parameters.

\begin{tabular}{|c|cc|}
\hline \hline Parameter & Value & Unit \\
\hline$m$ & 0.6 & $\mathrm{~kg}$ \\
$J$ & 0.014 & $\mathrm{kgm}^{2} / \mathrm{rad}$ \\
$a_{F}$ & 0.12 & $\mathrm{~m}$ \\
$a_{x}$ & -0.08 & $\mathrm{~m}$ \\
$k$ & 150 & $\mathrm{Nm} / \mathrm{rad}$ \\
\hline
\end{tabular}


The open-loop crossover frequency $\omega_{c}$, has a unique and direct relation with the bandwidth. The desired $\omega_{c}$ follows from the performance requirements. However, its maximum achievable value for obtaining a stable system might be restricted by the high-order modes of the plant. For instance, for the RMD, the rotational vibration mode, caused by the leaf spring, in the mode that restricts the maximum achievable bandwidth. This vibration mode occurs at the frequency of $\omega_{e}=62 \mathrm{rad} / \mathrm{s}$. Beyond the corresponding maximum achievable $\omega_{c}$, the motion loop enters the 'instability region'. The boundaries of the instability region depend greatly on the damping. Assuming a low damping, $\xi_{e} \leq 0.01$, in case of the RMD $\omega_{c}=10 \cdots 22 \mathrm{rad} / \mathrm{s}$ would yield an unstable MC loop $(\alpha=0.6)$. This follows directly from the guidelines presented in Chapter $\square$, specifically from Figure 2.5.

Measurements on the RMD lead to an estimated damping of the rotational vibration mode of $\xi_{e} \approx 0.04$, which is high enough for the RMD to remain stable in the aforementioned $\omega_{c}$ region. The main reasons for this high damping are the thick cables of the RMD, specially the cable of the linear actuator, and the dissipation due to the friction (two of the main sources of damping in mechanical structures (Rivin 1999)). The friction is mostly caused by asymmetric loading of the linear guideway. The friction ia partially compensated for by the addition of feedforward control in the motion-control loop.

Since the rotational vibration mode introduced by the leaf spring is rather isolated, the motion loop remains stable beyond the instability region corresponding the rotational vibration mode induced by the leaf spring (see Section 2.4.2). The higher-order modes of the RMD, appearing at relatively high frequencies, still put a limit on the achievable $\omega_{c}$. However, due to the typically present low-pass filter in the AVC loop (see Section 4.3.2), it is not possible to add active damping to those modes.

So adding damping will not result in increasing the achievable $\omega_{c}$, but can still increase the performance of the RMD by decreasing the settling time.

\subsection{Active damping}

Due to the stability robustness of collocated control, active damping has been implemented using collocated actuators and sensors. Applying an intrinsically passive control algorithm (Stramigioli 20001), which by definition is energetically passive or dissipative, in combination with collocated and dual actuator and sensor pairs, guarantees the stability of the control loop. Dual and collocated actuator and sensor pairs form an energy port between the controller and the plant. This can be realized by either the combination of force measurement and velocity actuation, or the combination of velocity measurement and force actuation.

For the RMD, use is made of piezo stacks (collocation) consisting of a force 


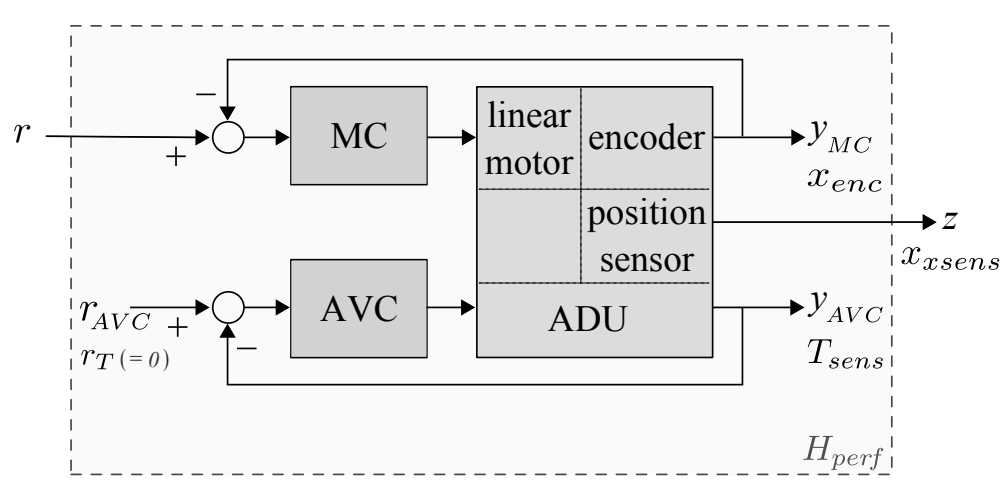

Figure 4.4: Control scheme of the plant in both motion control (MC) and active vibration control $(A V C)$ loop, including the various actuators, sensors and the relevant input and outputs.

sensor and position (displacement) actuator (duality). The passivity of this control has been addressd in Section B.3.T.

\subsubsection{Active vibration control}

The transfer function of the plant from the actuator of the AVC loop to its sensor for each vibration mode is given by:

$$
\left(\frac{T}{\varphi}\right)_{i}(s)=\frac{\phi_{i, A D U}^{2} k_{m, i} s^{2}}{s^{2}+\omega_{e, i}^{2}}
$$

The AVC algorithm, $C_{A V C}(s)$ (4.6), is the Leaking Integral Force Feedback (LIFF), which is an integral action, of which the pole is slightly shifted to the left of the origin (hence the term 'leaking') in combination with a low-frequent high-pass filter to avoid actuator saturation (Preumont 1997).

$$
C_{A V C}(s)=\frac{K_{L I F F}}{s+p_{L P}} \cdot \frac{s}{s+p_{H P}}
$$

The integral force feedback algorithm is intrinsically passive (see Section B.3.TI), but this does not apply for (4.6) because of the added filter. However, the stability is not endangered if the controller is tuned according to Holterman (20102):

$$
p_{L P} \leq 0.2 \omega_{e, 1} \quad p_{H P} \leq 0.2 p_{L P}
$$

Besides the increase of damping for the target resonance mode, this implementation of active damping also increases the damping of the higher-order modes of 
the plant that were not included in the reduced-order model (4.T). However, the extent of the resulting damping for the higher modes is unknown because of lack of information about these modes (एreumont 1997). The AVC controller is tuned optimally for the targeted rocking mode and might not add significant damping to the higher-order modes. Hence, these higher-order modes put an upper bound on the achievable bandwidth for motion control.

\subsubsection{Active damping unit}

The actuator and sensor of the AVC loop, possibly embedded in a mechanical structure, are termed the Active Damping Unit (ADU). To achieve sufficient damping, the ADU should fulfill the following requirements:

\section{Rotational actuation.}

Our objective is to add damping to the rotational vibration mode of the plant. Hence the ADU should be designed such that it affects the rotation of the end effector.

2. High stiffness.

Keeping the resonance frequency of the plant as high as possible benefits the performance. Hence the addition of the ADU should preferably not decrease the stiffness.

3. Actuated stroke proportional to the resonance amplitude.

It goes without saying that a good performance of the AVC loop depends, among others, on the capabilities of its actuator. To prevent nonlinearity caused by actuator saturation, the ADU should be designed such that its stroke is proportional to the resonance amplitude. This requirement also relates to the trade-off between the required stiffness, the available space and the allowable stresses in the mechanics of the ADU when in operation. The actuated rotation has been set to $0.70^{\circ}$.

\section{High controllability.}

The maximum achievable damping depends on the controllability of the resonance mode (Preumont 1997). So the ADU design should strive to achieve a high controllability. A system is said to be controllable if its controllability matrix, as defined by Kalman (1960) is full rank. For the collocated active damping loop, the controllability of each vibration mode is reflected in the distance between the corresponding pole and zero. By choosing proper ratios between the stiffnesses in the ADU, the distance between the pole and zero of the target mode can be maximized.

These requirements are translated in a mechanical design in what follows. 


\section{ADU model}

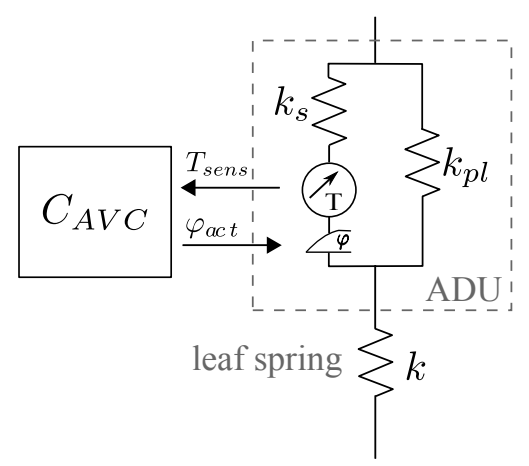

Figure 4.5: The lumped model of the ADU.

The stiffness's in the ADU can be modeled as two lumped stiffnesses; the stack stiffness, $k_{s}$, and the mechanism stiffness, $k_{p l}$, which can be represented as a stiffness parallel to the dynamic model of the stack, as shown in Figure 4.5. The stack stiffness is a property of the actuator and sensor used. The mechanism stiffness results from the hinges in the ADU ${ }^{\square}$. These stiffnesses together with the stiffness causing the rotational vibration mode (in this case the leaf spring), determine the controllability of the AVC loop, which is a measure for the maximum achievable active damping.

The transfer function of the active damping loop from the actuated angle to the measured torque is given by:

$$
P_{A V C}(s)=\frac{T_{\text {sens }}}{\varphi_{a c t}}(s)=g \cdot \frac{J s^{2}+d_{a} s+k_{a}}{J s^{2}+d_{e} s+k_{e}}
$$

where

$$
\begin{aligned}
g^{-1} & =\left(k_{p l}+k\right)^{-1}+k_{s}^{-1} \\
k_{a}^{-1} & =k_{p l}^{-1}+k^{-1} \\
k_{e}^{-1} & =\left(k_{p l}+k_{s}\right)^{-1}+k^{-1} \\
d_{\bullet} & =2 \xi_{\bullet} J \omega_{\bullet}
\end{aligned}
$$

The poles of $P_{A V C}, \omega_{e_{A V C}}$ (4.10), represent the resonance mode of the plant. Thus $\omega_{e_{A V C}}=\omega_{e}$. The resonance frequency of the subsystem constrained by the ADU

\footnotetext{
${ }^{2} k_{p l}$ is typical for mechanisms containing piezo's, as it can be used to apply a preload force to avoid tensile forces on the piezos
} 
actuator and sensor is the antiresonance of the AVC loop (the zeros), $\omega_{a_{A V C}}$ (4.TI) (Miu 11994).

$$
\begin{aligned}
\omega_{e_{A V C}} & =\sqrt{k_{e} / J} \\
\omega_{a_{A V C}} & =\sqrt{k_{a} / J}
\end{aligned}
$$

The larger the difference between $\omega_{e}$ and $\omega_{a_{A V C}}$, the more controllable the resonance mode is and hence the higher the maximum achievable damping, which, for a single mode, is equal to (Preumont 1997):

$$
\xi_{i}^{\max }=\frac{\omega_{e, i}-\omega_{a, i}}{2 \omega_{a, i}}
$$

The open-loop gain, $K_{o l}=g \cdot K_{L I F F}$, for which $\xi_{e, i}^{\max }$ can be obtained is (Preumont 1997):

$$
K_{o l}^{\xi^{\max }}=\omega_{e, i} \sqrt{\frac{\omega_{e, i}}{\omega_{a, i}}}
$$

Equation (4.9) shows that by choosing a low value for $k_{p l}$, it will become the dominant parameter in the equation for $k_{a}$. This results in a low $\omega_{a_{A V C}}$ and makes $k_{s}$ and $k$ together the dominant parameters that determine $\omega_{e}$. Therefore, $k_{p l}$ should be chosen as small as possible w.r.t. $k$ for a high controllability, and $k_{s}$ should be as large as possible to avoid decreasing $\omega_{e}$. The requirements for the ADU are $k_{p l}=9 \mathrm{Nm} / \mathrm{rad}$ and $k_{s}=80 \mathrm{Nm} / \mathrm{rad}$, which should result in $\omega_{e}=62$ $\mathrm{rad} / \mathrm{s}$ and $\omega_{a_{A V C}}=25 \mathrm{rad} / \mathrm{s}$, according to the dynamical model.

Note that $k_{s}<k$, which means that the plant stiffness decreases considerably due to the presence of the ADU, which contradicts requirements number 2 . The relatively low value of $k_{s}$ is because the ADU was designed for an earlier version of the RMD which had a more compliant leaf spring, namely $k=50 \mathrm{Nm} / \mathrm{rad}$. Measurements on this realization of the RMD showed a higher antiresonance frequency than expected, making the difference between the resonance and antiresonance frequency of the first mode much smaller than intended by the ADU design. One of the possible explanations was a higher $k_{p l}$ in the realized ADU compared to the design, for instance due to thicker hinges. If so, the controllability would increase by increasing $k$. Increasing $k$ results in an increase of both $k_{e}$ and $k_{a}$ (4.9), but to different extents. Consequently, $\omega_{e}$ increases more than $\omega_{a}$, yielding a higher controllability. So the original leaf spring was replaced by the current one, for which $k=150 \mathrm{Nm} / \mathrm{rad}$.

Replacing the leaf spring did not have the desired effect, and so it excluded the investigated possibility as an explanation for the low controllability. Another consequence of replacing the leaf spring is that the resulting increase of $\omega_{e}$ allows for a higher bandwidth, which is favorable in light of the high friction in the RMD. So it was decided to keep the new leaf spring, but time constraints prevented the redesign of the ADU to match $k_{s}$ and $k$ as desired. 


\section{Mechanical design of the ADU}

On the basis of the deduced requirements for the ADU, a flexure mechanism has been designed by Dr. ir. D. M. Brouwer and Imotec b.v. (Holterman 20T0), in which two piezo actuator-sensor stacks are embedded (see Appendix $\triangle$ for the detailed design). An integrated preload adjustment screw assures that the piezo is loaded compressively at all times. A three-dimensional view of this mechanism is shown in Figure 4.6 .

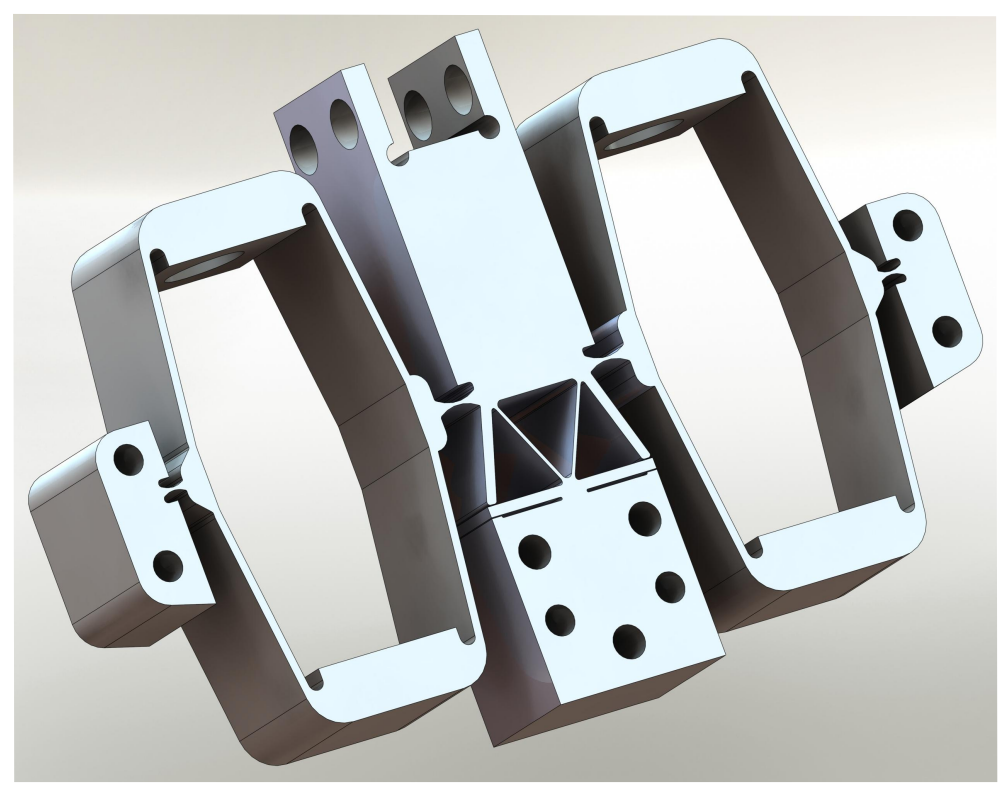

Figure 4.6: 3D view of the Active Damping Unit.

This mechanism transforms the $20 \mu \mathrm{m}$ translational motion of the piezo stacks into a $0.70^{\circ}$ rotation. To obtain the required transformation ratio, the stroke of the piezo stacks should be amplified. This is realized by means of a range amplification mechanism (elliptic shell) as shown in Figure 4.7 (Holterman \& Groen 2012). The resulting stroke amplification, $g_{\text {shell }}$, (calculated in Appendix is 6.9 .

The transformation of the amplified translational stroke to rotation is performed by a main flexure. The main flexure hinge consists of two leaf springs, as shown in Figure 4.6. The intersection of the leaf springs creates an instant center of rotation, creating a compliant degree of freedom around the $z$-axis. The other five degrees of freedom are stiffly supported. In terms of stiffness, these leaf springs act in parallel. Hinges 2 and 3 are mainly loaded axially, and therefore these can conveniently be designed as notch hinges. The longitudinal stiffness of 


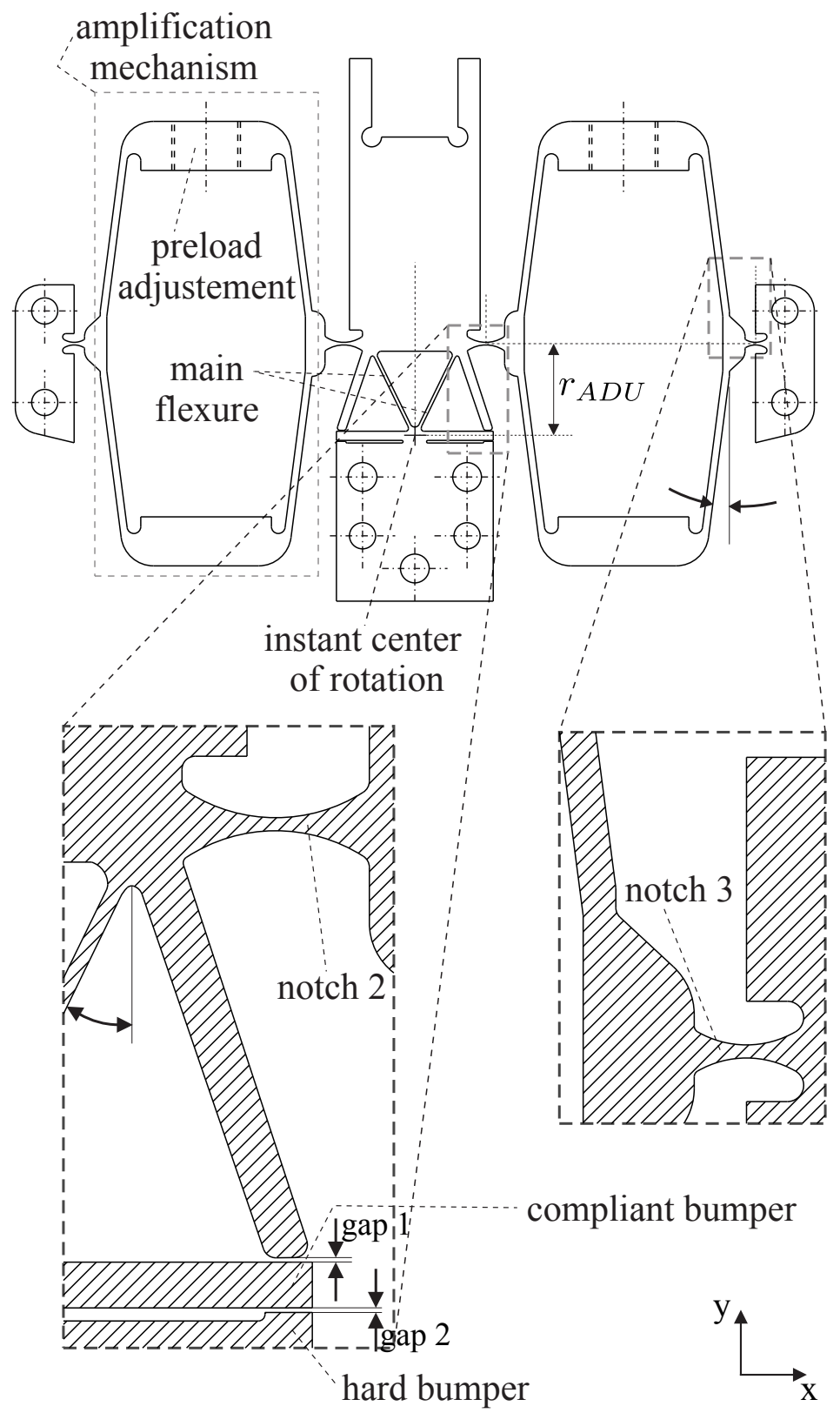

Figure 4.7: Drawing top view of the Active Damping Unit. 
the notch hinges and the stiffness in $x$-direction of the main flexure are several orders higher than stiffness of the stack and hence can be disregarded. So $k_{s}$ is mostly determined by stack stiffness. The stackstiffness of two stacks is calculated to be $98.5 \mathrm{Nm} / \mathrm{rad}$.

A FEM model however showed a stroke amplification factor of $g_{\text {shell }}=6.3$ and a stiffness of $k_{s}=82 \mathrm{Nm} / \mathrm{rad}$. The difference is caused by the parasitic stiffnesses in the FEM model which add extra compliance.

The combined rotational stiffness of the flexure mechanism $k_{p l}$, without piezo's and piezo amplification mechanism is a combination of the stiffness of the main flexure, and notch hinges 2 and 3 . With the dimensions listed in Appendix 因, $k_{p l}$ becomes $9.2 \mathrm{Nm} / \mathrm{rad}$. Table 4.2 shows an overview of the relevant parameters of the ADU.

Table 4.2: The parameters of the ADU.

\begin{tabular}{|c|cc|}
\hline \hline Parameter & Value & Unit \\
\hline$k_{p l}$ & 9.2 & $\mathrm{Nm} / \mathrm{rad}$ \\
$k_{s}$ & 82 & $\mathrm{Nm} / \mathrm{rad}$ \\
$g_{\text {shell }}$ & 6.3 & - \\
$r_{A D U}$ & $6.5 \mathrm{e}-3$ & $\mathrm{~m}$ \\
\hline \hline
\end{tabular}

To protect the flexure from overloading by the actuators, a compliant bumper is introduced, as depicted in Figure 4.7. When the rotation exceeds the allowable value, gap 1 is reduced to zero and the compliant bumper is collided with. The stiffness of this bumper is ten times the rotational stiffness of the flexure. This way the collision forces are limited. After running through gap 2 a second bumper with large stiffness stops the rotational motion entirely.

\section{ADU in RMD}

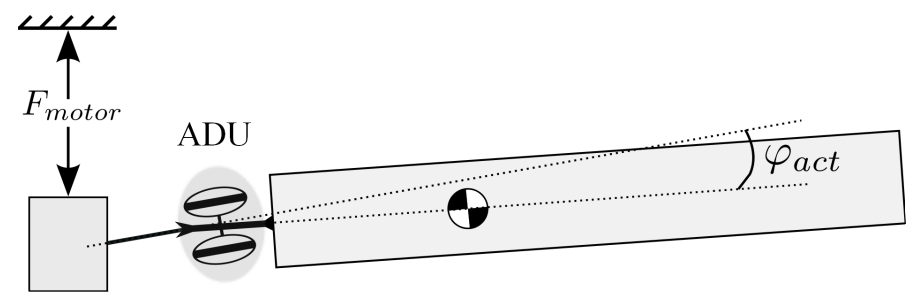

Figure 4.8: Schematic top view of the ADU in RMD.

Figure 4.8 shows a schematic representation of Figure 4.2 . The torque between the leaf spring and the bar is measured in the form of two equal linear forces 
in opposite directions, by the piezo force sensors; $F_{\text {sens } 1}=-F_{\text {sens } 2}$. The transformation ratio between the measured force and the corresponding torque is $r_{A D U}$. The differential actuation of piezoelectric actuators results in the desired angle, $\varphi_{\text {act }}$, determined by the active damping controller.

$\boldsymbol{A}_{\text {sens }}$ and $\boldsymbol{A}_{\text {act }}$ (see Figure 4.9) incorporate the transformation ratios $r_{\text {sens }}$ and $r_{a c t}$. These ratios transform the $C_{A V C}$ input and output signals between the rotational and translational domain, the electrical and mechanical domain, and the modal and local plant coordinates;

$$
\begin{aligned}
\boldsymbol{A}_{\text {sens }} & =r_{\text {sens }} \cdot\left[\begin{array}{ll}
1 & -1
\end{array}\right]^{T} \\
T_{\text {sens }} & =\boldsymbol{A}_{\text {sens }}\left[\begin{array}{ll}
F_{\text {sens } 1} & F_{\text {sens } 2}
\end{array}\right]^{T} \\
\boldsymbol{A}_{\text {act }} & =r_{\text {act }} \cdot\left[\begin{array}{ll}
1 & -1
\end{array}\right] \\
{\left[\begin{array}{ll}
x_{\text {act } 1} & x_{\text {act } 2}
\end{array}\right]^{T} } & =\boldsymbol{A}_{\text {act }} \varphi_{\text {act }}
\end{aligned}
$$

The ADU is controlled under the assumption that the structure is symmetrical, which means that the transfer functions between the two actuators and sensors are equal. Measurements show that this is indeed the case for the realized ADU.

The finite element model of the ADU mechanics predicts $\omega_{a_{A V C}}=42 \mathrm{rad} / \mathrm{s}$. This means that the stiffnesses in the ADU that form the lumped parameter $k_{p l}$ in the dynamical model, are not as anticipated. An explanation could be that modeling the elliptic shell as a linear stiffness (and stroke amplifier) does not give a competent description of its dynamics.

The measurements show a resonance mode at $\omega_{e}=62 \mathrm{rad} / \mathrm{s}$ as expected from the plant parameters. However, the measured antiresonance frequency of $\omega_{a_{A V C}}=55 \mathrm{rad} / \mathrm{s}$ is much higher than predicted by both the dynamical model and the finite element model of the RMD including the ADU. The reason for this significant discrepancy has not been found yet $t^{\text {B. }}$.

The combination of the measured $\omega_{e}$ and $\omega_{a_{A V C}}$ leads to $\xi^{\max }=0.06$, which is significant in terms of motion loop stability, but is quite low in terms of achieving a well-damped transient response.

Furthermore, the measured transfer function shows a $1^{s t}$-order low-pass filter $H_{f l t}$ (see Figure 4.10 ), with a cut-off frequency of around $\omega=170 \mathrm{rad} / \mathrm{s}$ in the actuator amplifier that is currently available in our laboratory. This filter limits the active damping of the higher-order modes. 


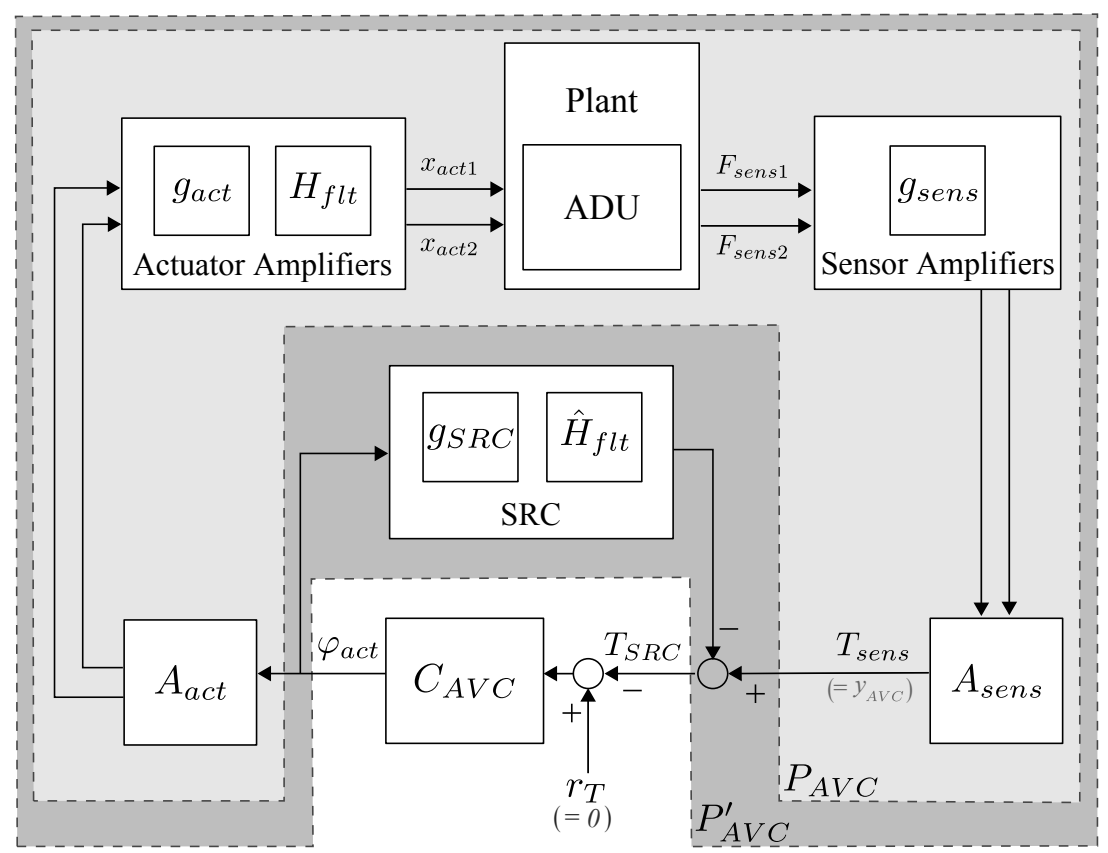

Figure 4.9: AVC loop including static response compensation.

\subsubsection{Static response compensation}

To increase the controllability of the AVC loop, Static Response Compensation (SRC) can be performed. SRC will shift the antiresonance frequency of the vibration mode towards the origin, by accumulating an inverse stiffness to the plant's response in a parallel path, termed the compensation path, as shown in Figure 4.9. This is basically the same idea as 'feedthrough compensation' presented by Holterman \& de Vries (20(1)4) and implemented among others by Aphale et al. (20)7).

The gain of the compensation path, $g_{S R C}$, should ideally be equal to the static response level (low-frequency gain) to obtain the maximum achievable controllability.

$$
\begin{aligned}
g_{S R C} & =\hat{g} \cdot \hat{k}_{a} / \hat{k}_{e} & & \text { static response level estimation } \\
g_{S R C, i d l} & =g \cdot k_{a} / k_{e} & & \text { ideal situation }
\end{aligned}
$$

\footnotetext{
${ }^{3}$ For further analysis, measurements directly on the flexure mechanism and piezo stacks should be performed. However, the mechanism is not accessible anymore due to metal sheets glued both on top and bottom, connecting the rotational fixed worlds, and the piezos are in turn glued to the flexure mechanism.
} 
An inverse stiffness increasing beyond the static response level of the AVC loop moves the zeros belonging to the antiresonance along the real axis of the s-plane to infinity, after which they come back on the imaginary axis. The latter situation is also referred to as 'pole-zero flipping' ([reumont 1997). Thus $g_{S R C}>g_{S R C, i d l}$ endangers the stability of the AVC loop.

Starting from (4.8), the compensated AVC loop transfer function can be deduced as follows:

$$
\begin{aligned}
& P_{A V C}^{\prime}(s)=P_{A V C}(s)-g_{S R C} \\
& =g \cdot \frac{J s^{2}+d_{a} s+k_{a}}{J s^{2}+d_{e} s+k_{e}}-\hat{g} \cdot \frac{\hat{k}_{a}}{\hat{k}_{e}} \\
& =\frac{\left(g-\hat{g} \frac{\hat{k}_{a}}{\hat{k}_{e}}\right) J s^{2}+\left(g d_{a}-\hat{g} \hat{k}_{a}\right.}{J s_{e}+d_{e} s+k_{e}} s+\left(g k_{a}-\hat{g} \hat{⿳}_{\hat{k}_{e}} k_{e}\right)
\end{aligned}
$$

Assuming $g_{S R C}=r_{c} \cdot g_{S R C, i d l}$ with $0 \leq r_{c} \leq 1$ :

$$
P_{A V C}^{\prime}(s)=g \cdot \frac{\left(1-r_{c} \frac{k_{a}}{k_{e}}\right) J s^{2}+\left(d_{a}-r_{c} \frac{k_{a}}{k_{e}} d_{e}\right) s+\left(1-r_{c}\right) k_{a}}{J s^{2}+d_{e} s+k_{e}}
$$

The transfer function of $P_{A V C}^{\prime}$ shows that if $d_{e}>d_{a} \cdot k_{e} / k_{a}$, SRC can result in non-minimum phase zeros. In addition, the fact that the non-linear behavior of piezo (hysteresis) is compensated by a linear stiffness can lead to non-minimum phase zeros.

The compensation path should also contain an estimate of the additional dynamics in the frequency region of interest, in this case the low-pass filter $\hat{H}_{f l t}$.

The measured Frequency Response Function (FRF) of the compensated loop, from the output of $C_{A V C}$ (indicated by $\phi_{a c t}$ in Figure 4.9) to $T_{S R C}$, is depicted in Figure 4.10 for various values of $r_{c}$. The phase plot shows that the zeros of the AVC loop not only shift towards the origin as $r_{c}$ increases, they also exhibit non-minimum phase behavior. This could be caused by the non-linearity of the piezo elements, as well as the fact that $\xi_{e} \approx 0.04$ and $\xi_{a} \approx 0.02$, thus $\xi_{e}>\xi_{a}(\xi$ relates to $d$ according to (4.9)). The consequence of non-minimum phase zeros is that for an increasing $K_{L I F F}$, depending on the location of AVC zeros, either the resonance poles, or the AVC poles, first approach the imaginary axis, and for even higher values of $K_{L I F F}$ eventually enter the right half-plane and become unstable. Nonetheless, this is not expected to jeopardize the stability of the AVC loop, since the open-loop gain of the AVC loop should be tuned such that $K_{o l} \leq$ $K_{o l}^{\xi_{\text {max }}}$. The maximum achievable active damping however, will decrease slightly by the shift of the zeros to the right half of the complex plane.

Because of the non-minimum phase zeros of the AVC loop and the presence of the low-pass filter, the rules for the achieving maximum damping, (4.12) and 

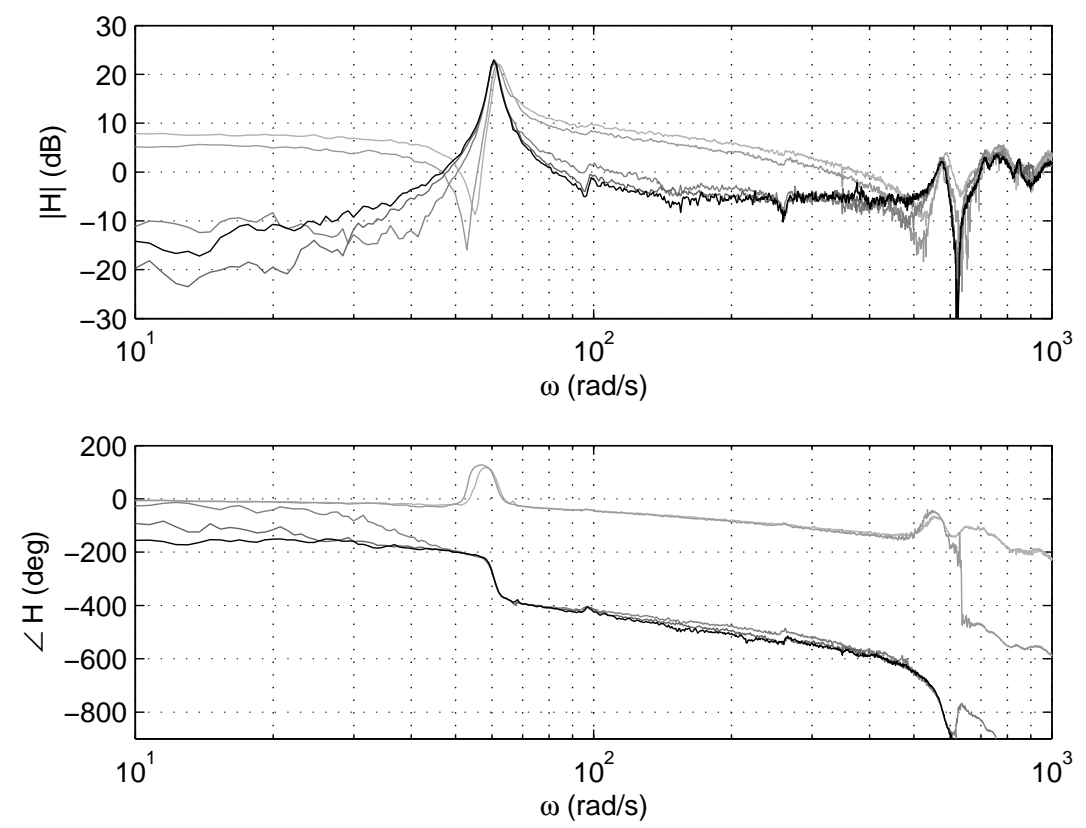

Figure 4.10: The measured bode plot of the open AVC loop, without SRC (light gray), and the resulting bode plots after SRC for various ratios of $g_{S R C}$. The shown ratios are, from light gray to black: 0, 0.2, 0.8, 1 .

(4.T3), are not exact anymore. The gain of the AVC loop can be tuned using the measured open-loop transfer function shown in Figure 4.71 , and the optimal $K_{L I F F}$ can be chosen using the closed-loop transfer function.

\subsection{Measurements}

Figure $4 . T$ shows the frequency response functions from $r_{A V C}$ to $T_{R S C}$ (see Figure 4.9) for both open and closed AVC loop. Figure 4.T2 shows the same for the transfer function from $r_{A V C}$ to $T_{\text {sens }}$, which is the not compensated transfer functions of the AVC loop. The value of $K_{L I F F}$ is first estimated using the measured FRF of the open AVC loop after SRC, as shown in Figure 4.T0 (ratio of $g_{S R C}$ is equal to one). Then, the estimated $K_{L I F F}$ is tuned on the basis of the closed-loop FRF, and time measurements, using the magnetic position sensor. The increased damping of the resonance mode is clearly visible in both plots by the flattened resonance peak. Using the magnification-factor method (de Silva 

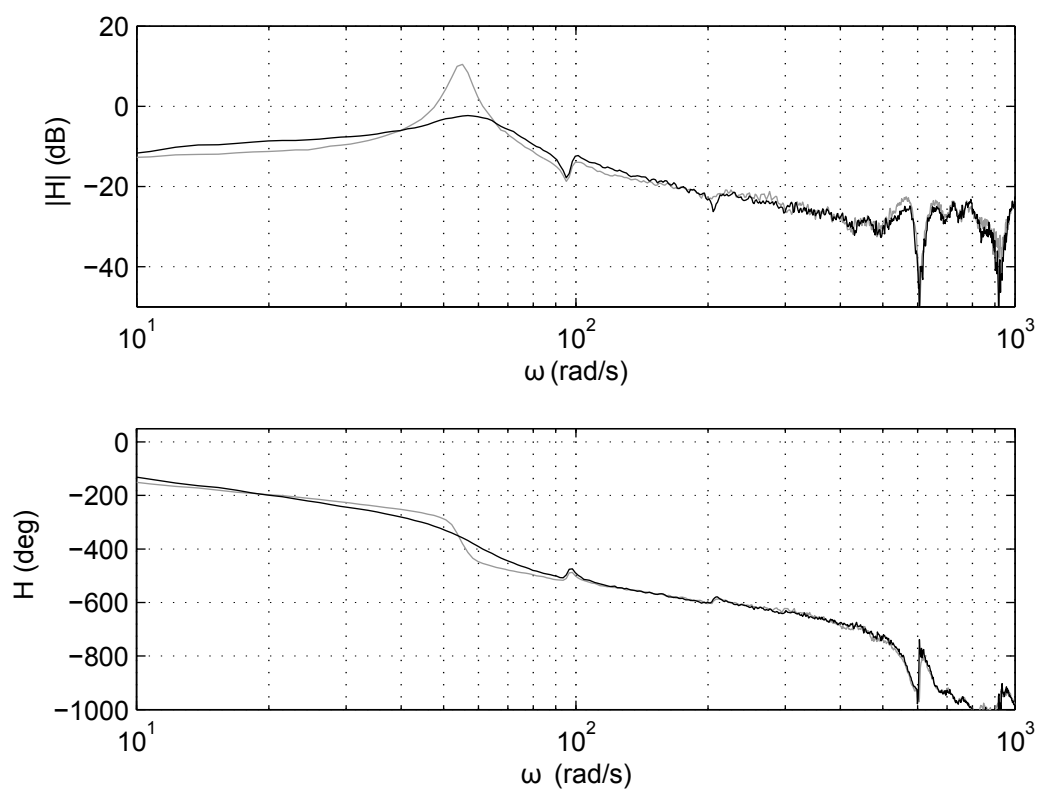

Figure 4.11: The measured bode plot of AVC loop, from $r_{A V C}$ to $T_{R S C}$, with SRC, gray: open loop, black: closed loop; $K_{L I F F}=30$.
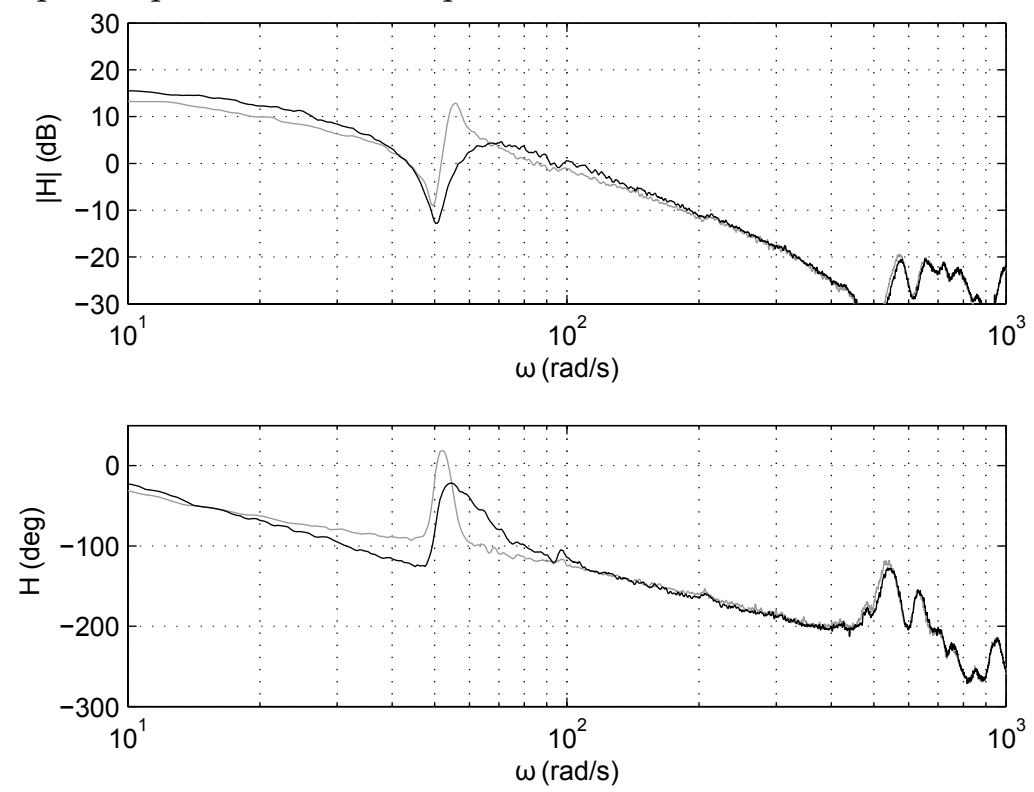

Figure 4.12: The measured bode plot of AVC loop, from $r_{A V C}$ to $T_{\text {sens, }}$ with SRC, gray: open loop, black: closed loop; $K_{L I F F}=30$. 
2000) and curve fitting, the increased modal damping is estimated to be $\xi_{e}=0.2$. By tuning both the SRC parameters and $K_{L I F F}$, the achieved active damping could be further increased. The SRC gain can be used for varying the location of the zeros belonging to the AVC loop (tuning the real part versus the imaginary part). By adjusting $K_{L I F F}$ at the same time, the achieved damping could be maximized. However, striving for the maximum possible damping decreases the stability robustness of the AVC loop, due to the presence of the non-minimum phase zeros.
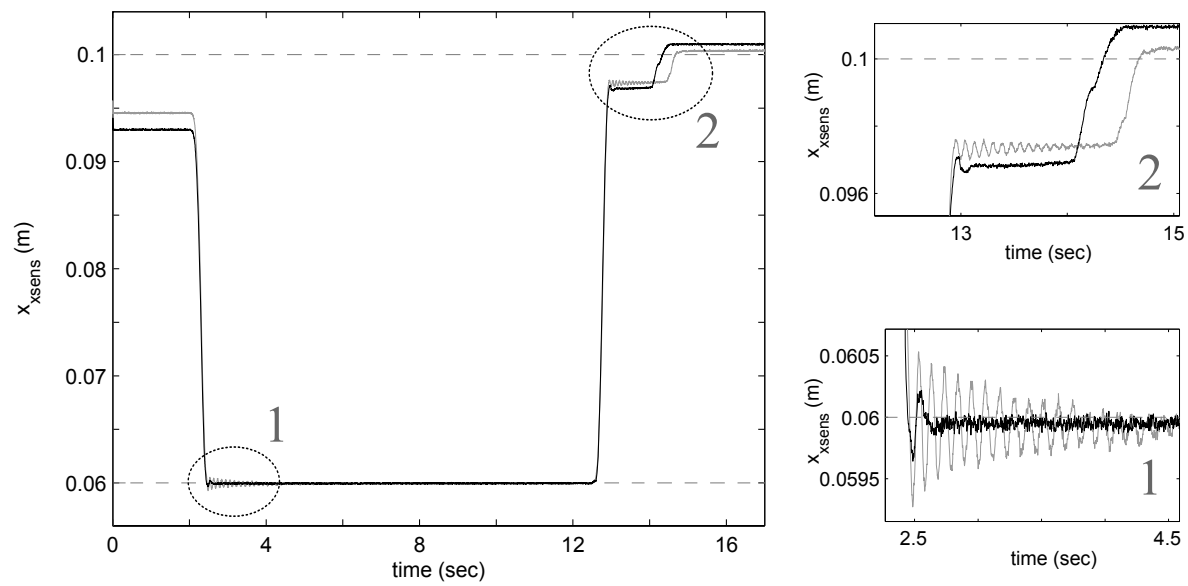

Figure 4.13: Position measurement, $x_{x s e n s}(\mathrm{~m})$ v.s. time $(s), \omega_{c}=20 \mathrm{rad} / \mathrm{s}$, gray: no AVC, black: with $A V C, K_{L I F F}=30$. The motion profile starts 2 seconds after an open-loop homing. The dashed lines show the set-points that the third-order motion profile is designed to reach.

The resulting performance improvement can be seen from the measured position at the tip of the bar $x_{x s e n s}$, in Figure 4.13. This figure shows the reduction of the settling time resulting from the implemented active damping. Both measurements (with and without AVC) are performed after a homing procedure. The homing procedure is open loop, which results in different starting position of the end effector for each measurement; $x_{e n c}(0) \approx 0.09 \mathrm{~m}$. The motion profile starts after 2 seconds. The enlarged post-transition position measurements are shown on the right. In the top zoomed-in plot, the effect of (static) friction on the performance is clearly visible. Although the effect of friction is the main performance-limiting factor in this case, the improvement in the transient response resulting from active damping is evident from these measurements. 


\subsection{Conclusions}

Collocated active damping has been implemented in a test setup suffering from a rotational vibration mode. As a result of the implemented active damping, the relative damping of the rotational vibration mode of the setup was increased by a factor five. The beneficial effect of the increased damping is clearly visible in the position of the end effector.

The controllability of the rotational vibration mode with the current active damping unit is low. To apply sufficient damping, the controllability has been increased by compensating the static term of the loop transfer. This compensation leads to non-minimum phase zeros, decreasing the stability robustness of the active damping loop. However, it enables a relative damping that is three times higher than the theoretical maximum achievable damping without compensation.

The physical damping that is present in the test setup is already high enough, such that the bandwidth limitation of low damping does not apply. 


\section{Chapter 5}

\section{Off-axis Modal Active Vibration Control}

Abstract - Collocated Active Vibration Control (AVC) is an effective and robustly stable way of adding damping to the performance limiting vibrations of a plant. Although the active damping unit containing the collocated actuator and sensor belonging to the AVC loop is designed to enable modal active damping, parameter variations can result in a mismatch between the active damping axis and the vibration axis. This chapter presents a model-based evaluation of the interaction between the AVC and the plant. The effect of the deviation between the active damping axis and the vibration axis on the dynamics of the end effector is shown in simulation.

This chapter is a major revision of (Babakhani, de Vries \& van Amerongen 2012d); "Off-axis modal active vibration control of roational vibrations"

B. Babakhani and T. J. A. de Vries and J. van Amerongen

Proceedings of the IEEE/ASME International Conference on Advanced Intelligent Mechatronics, Taiwan, July 11-14, 2012. 


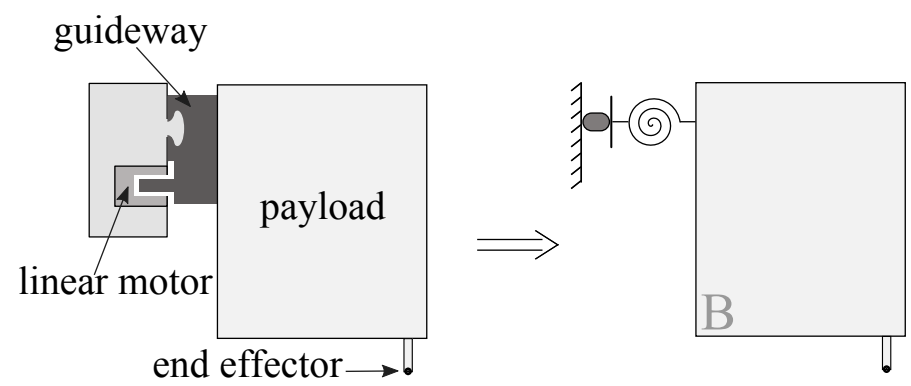

Figure 5.1: Schematic representation of a motion system fitted with a linear drive. The simplified version, shown on the right side, is used in this chapter to represent the plant.

\subsection{Introduction}

Vibrations of industrial machines can be considered an important performance limiting factor. These inevitable dynamics are caused by compliances and masses in the machine. The effect of vibrations can be minimized by increasing the damping. This can be realized when designing the mechanical structure of the machine (Rivin 1999) or by adding passive damping (mechanical) (Mead 1998). When these measures turn out to be insufficient or costly, active means can be implemented to inject damping in the vibration modes (thus, adding a mechatronic damper). Collocated active damping in combination with an energetically passive controller is a suitable option, mainly because of its inherent robustness w.r.t. stability (Preumont 1997). For this, a collocated actuator and sensor pair have to be added to the plant.

The mechanism incorporating this collocated pair, termed Active Damping Unit (ADU), is designed on the basis of the vibration problem at hand. Both the 'shape' and the frequency of the vibrational mode(s) are considered when designing the degree(s) of freedom of such mechanisms. However, in practice the vibration axis can change, for instance due to parameter variations in time or load variations during operation. This will result in a deviation between the vibration axis, and the axis of active vibration control, which we term as 'off-axis' vibration control.

In this chapter, the effect of off-axis active vibration control on the plant dynamics and the achieved damping is treated. The studied case is a motion system fitted with a linear drive. Such machines, also called "flexible guidance" class of plants, generally suffer from rotational vibrations around their guideway (Coelingh et al. 2002, Babakhani \& de Vries 2010a) (see Figure 5.I). Using the model of such a plant, the effect of active damping for both off-axis and direct modal control is discussed. 


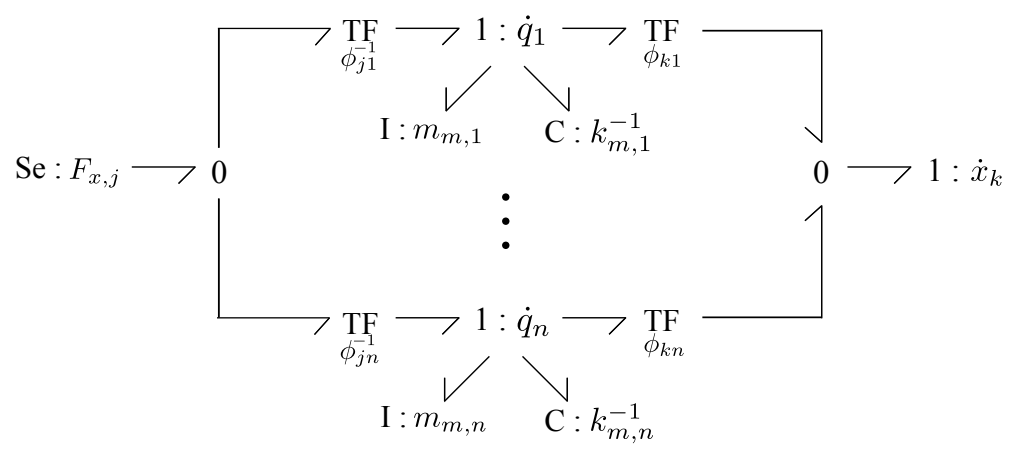

Figure 5.2: Modal decomposition of the transfer function (5.d) in bond graph representation.

In Section 5.2, a model of the plant considered here is given in modal terms (see Section [2.2), which includes the rigid body mode of the plant and one rotational vibration mode (the dominant mode). Modeling in modal coordinates is chosen, because then the system can be described by a set of decoupled differential equations.

Though a modal control algorithm is chosen for Active Vibration Control (AVC), ADU is described more conveniently in terms of Euclidean coordinates. Hence, to model the interaction between the plant and the ADU, another modeling method has been used, which is based on the screw theory (see Ball 1900). The principles of this method are explained in Section 5.3.1. Next, the 3D model of the plant is presented in Section [5.3.2.

Section 5.4 deals with active damping. The model of the ADU and its effect on the end-effector position of the payload are discussed in Section 5.4.2. This is done on the basis of the ADU location w.r.t. the compliance present in the plant. The obtained models are represented in bond graphs. The reader is referred to Karnopp et al. (2006) and Breedveld (1985) for more information on bond graphs and multibond graphs. Section 5.5 presents the simulation results. The conclusions can be found in Section 5.6 .

\subsection{Modal model of vibrations}

A modal model of the plant in modal terms is assumed to be given by 2.9, which is repeated here for convenience:

$$
\frac{x_{k}}{F_{x, j}}(s)=\sum_{i=1}^{n} \frac{\phi_{i j} \phi_{i k}}{m_{m, i}(s)^{2}+k_{m, i}}
$$




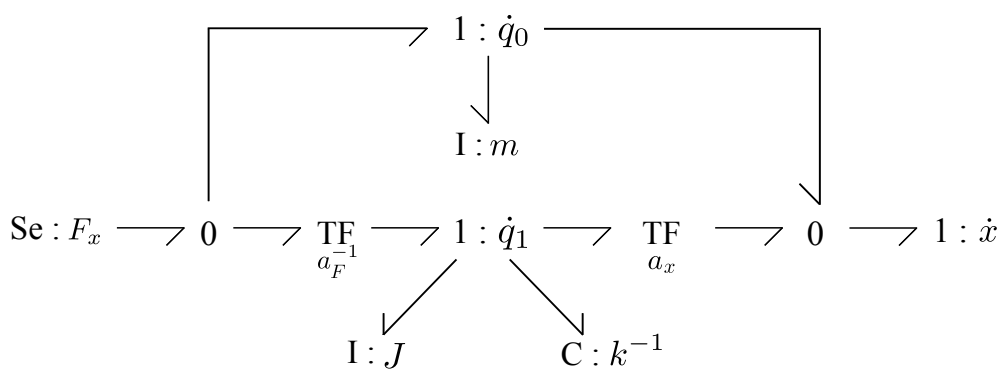

Figure 5.3: The bond graph representation of a 1D modal model of the flexible guidance.

Here, $m_{m, i}$ and $k_{m, i}$ respectively represent modal mass and stiffness of the $i$-th mode. Furthermore, the resonance frequency of the $i$-th vibration mode, $\omega_{e, i}$, is given by:

$$
\omega_{e, i}=\sqrt{\frac{k_{m, i}}{m_{m, i}}}
$$

The bond graph of this modal model is shown in Figure 5.2. Here, I and $C$ represent the modal inertia and modal compliance respectively. The elements of the mode-shape vectors are modeled as transformation ratios of the transformers, $\mathrm{TF}$, the 1-junctions represent the common flow (generalized and modal velocities) nodes and the 0 -junctions stand for common effort (generalized and modal forces) nodes. A one-dimensional (1D) model of the flexible guidance incorporating the rigid body mode, $i=0\left(k_{m, 0}=0\right)$, and one vibration mode, $i=1$, was presented in Section 2.5 in terms of effective modal parameter (2.25), which is repeated here for convenience (damping has been omitted).

$$
\begin{aligned}
\frac{x}{F_{x}}(s) & =\frac{1}{m s^{2}}+\frac{a_{F} a_{x}}{J s^{2}+k} \\
& =\frac{1+\alpha}{m s^{2}} \cdot \frac{s^{2}+\omega_{a}^{2}}{s^{2}+\omega_{e}^{2}}
\end{aligned}
$$

The bond graph representation of this model can be composed using Figure 5.2. The resulting model is depicted in Figure 5.3, which can also be found in (Coelingh et al. 2002).

\subsection{D dynamical model of the plant}

In this section, the effect of the modeled rotational vibration mode on the 3D dynamics of the end effector is included in the model. The dynamics of the 
plant are modeled in modal coordinates (see Section 5.2). Interaction with the ADU is modeled using the screw theory. The advantage of this approach is that geometrical properties of the plant can be used to easily derive the transformation ratios that determine the dynamics of the system. The basics of these methods are explained in what follows. The link to the modal model (2.8), is shown by means of a few examples. The theory used in this section is based on (Stramigioli \& Bruyninckx 2007) and (Visser et al, 2009).

\subsubsection{Rigid-body motion}

Though the payload is typically not completely (or infinitely) rigid, its dynamics can usually be disregarded compared to the effect of the compliance in the guideway, which causes relatively low-frequency vibrations of the payload.

The screw theory states that the motion of a rigid body can be described as a rotation around an axis and a translation parallel to that same axis, the so-called screw (Ball 1900).

Each body is defined to have its own body-fixed coordinate frame $\Psi$, expressed in the Cartesian coordinates $x, y$ and $z$. The twist vector, $T$ (5.4), gives a coordinate-free representation of the velocity of a body, which consists of rotational $(\omega)$ and linear $(v)$ velocities ${ }^{\mathrm{m}} . T_{i}^{k, j}$ is the geometrical velocity of the body fixed to $\Psi_{i}$ w.r.t. $\Psi_{j}$, expressed in $\Psi_{k}$.

$$
\begin{aligned}
T_{i}^{k, j} & =\left[\begin{array}{ll}
\omega & v
\end{array}\right]^{T} \\
& =\left[\begin{array}{llllll}
\omega_{x} & \omega_{y} & \omega_{z} & v_{x} & v_{y} & v_{z}
\end{array}\right]^{T}
\end{aligned}
$$

The force and torque vectors acting on a body form the wrench vector, $W$. $W^{i}$ (5.5) is a wrench expressed in $\Psi_{i}$.

$$
\begin{aligned}
W^{i} & =\left[\begin{array}{ll}
\tau & F
\end{array}\right] \\
& =\left[\begin{array}{llllll}
\tau_{x} & \tau_{y} & \tau_{z} & F_{x} & F_{y} & F_{z}
\end{array}\right]
\end{aligned}
$$

The coordinate transformation from $\Psi_{i}$ to $\Psi_{j}$ is defined by the homogeneous matrix $H_{i}^{j}$ :

$$
H_{i}^{j}=\left[\begin{array}{cc}
R_{i}^{j} & p_{i}^{j} \\
O_{3}^{T} & 1
\end{array}\right]
$$

where $p_{i}^{j}$ represents the coordinates of the vector from $o_{\Psi_{j}}$ (the origin of $\Psi_{j}$ ) to $o_{\Psi_{i}}$ (the origin of $\Psi_{i}$ ), expressed in $\Psi_{j} . R_{i}^{j}$ is a $3 \times 3$-matrix describing the rotational

\footnotetext{
${ }^{1}$ Note that when comparing $T$ with $\dot{\boldsymbol{x}}(t)$ (see Section $\mathbf{b . 2}$ ), the rotational velocity, $\omega$, is the same as the angular velocity of the body, but the linear velocity, $v$, is a projection of the translational and angular velocity of the object, passing through the origin of the frame in which the twist is expressed.
} 
transformation from $\Psi_{i}$ to $\Psi_{j}$. Furthermore,

$$
H_{j}^{i}=\left(H_{i}^{j}\right)^{-1}
$$

and

$$
H_{n}^{0}=H_{1}^{0} H_{2}^{1} \cdots H_{n}^{n-1}
$$

The coordinate transformation of an arbitrary point in $\Psi_{i}, P^{i}=\left[\begin{array}{lll}x & y & z\end{array}\right]^{T}$, to $\Psi_{j}$ is performed by:

$$
\left[\begin{array}{c}
P^{j} \\
1
\end{array}\right]=H_{i}^{j}\left[\begin{array}{c}
P^{i} \\
1
\end{array}\right]
$$

and the following holds for the twist and wrench vectors:

$$
\begin{gathered}
T_{*}^{j, *}=A d_{H_{i}^{j}} T_{*}^{i, *} \\
\left(W^{i}\right)^{T}=A d_{H_{i}^{j}}^{T}\left(W^{j}\right)^{T}
\end{gathered}
$$

where the Adjoint of $H_{i}^{j}$ is given by:

$$
A d_{H_{i}^{j}}=\left[\begin{array}{cc}
R_{i}^{j} & 0 \\
\tilde{p}_{i}^{j} R_{i}^{j} & R_{i}^{j}
\end{array}\right]
$$

$\tilde{P}$ denotes the skew symmetric matrix of $P$, which is defined by:

$$
\tilde{P}=\left[\begin{array}{ccc}
0 & -z & y \\
z & 0 & -x \\
-y & x & 0
\end{array}\right]
$$

\subsubsection{The plant model using the screw theory}

In this section, the relevant parameters describing the interaction between the plant and the ADU, together with the mode-shape vectors relating the modeled vibration mode to the end-effector position, are deduced using the screw theory.

Figure 5.4 depicts the first rotational vibration mode of the plant. The payload, $\mathrm{B}$, is connected to the fixed world by a compliant body, the guideway, shown with a rotational-spring icon. The guideway can be modeled as a modal stiffness, $k$. The fixed world is symbolized by the global coordinate frame $\Psi_{0}$. Also the assumed axis of rotation (the $z$-axis of $\Psi_{D}$ ) is shown. For ease of explanation, let's assume that the $z$-axis of $\Psi_{D}$ is parallel to the $z$-axis of $\Psi_{0}$. Since the ADU only interacts with the end effector (which means that its dynamics are analyzed only w.r.t. $\Psi_{m}$ and not $\Psi_{0}$ ), this assumption does not affect the analysis. The $z$-axis of the coordinate frame of the payload, $\Psi_{m}$, is aligned with the modal axis. The 


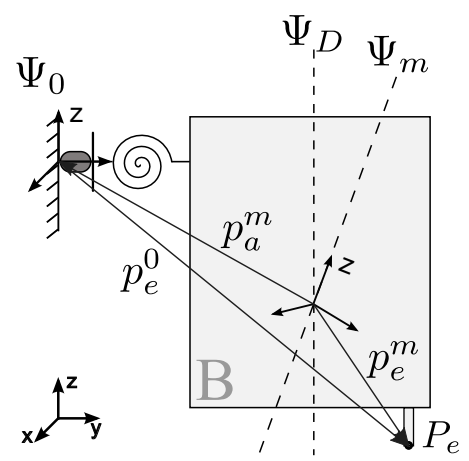

Figure 5.4: Schematic representation of the plant (B) vibrating around its modal axis (skew dashed line) due to the compliant guideway, shown symbolically by a rotational-spring icon. Also shown in this picture are the global coordinate frame (fixed world) $\Psi_{0}$, the body coordinate frame $\Psi_{m}$, the $z$-axis of which is aligned with the modal axis, and the axis around which the active damping is applied, which is the z-axis of $\Psi_{D}$. The end effector is indicated by $P_{e}$.
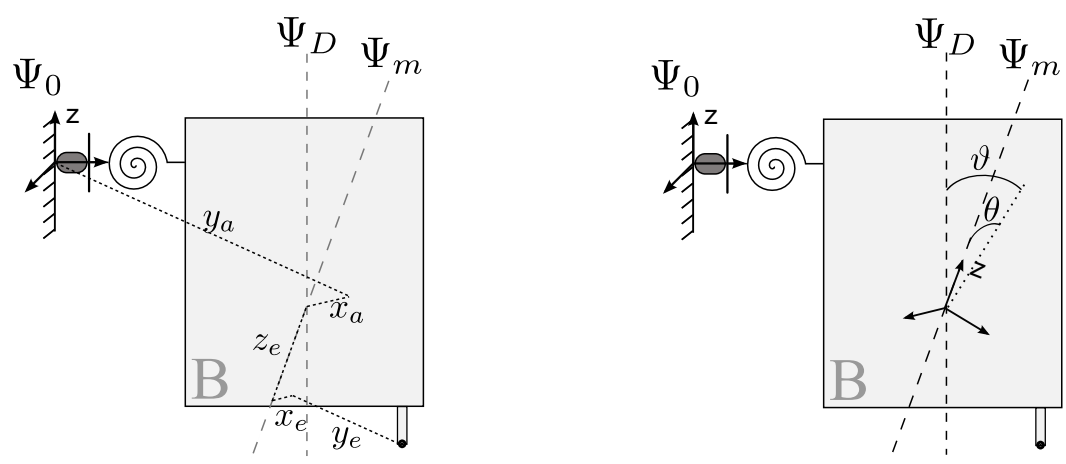

Figure 5.5: Schematic representation of the plant (B) vibrating around its modal axis (skew dashed line). Shown in this picture are the coordinates of both the modal axis $\left(x_{a}, y_{a}\right.$ and $\left.z_{a}\right)$ and end effector $\left(x_{e}, y_{e}\right.$ and $\left.z_{e}\right)$, and the angles $(\vartheta$ and $\theta$ ) by which $\Psi_{m}$ and $\Psi_{D}$ are rotated with respect to each other. The $z$-axes of $\Psi_{0}$ and $\Psi_{D}$ are parallel to each other. 
modal axis, around which the payload 'B' vibrates (the $z$-axis of $\Psi_{m}$ ), is rotated w.r.t. the assumed axis of rotation by an angle $\vartheta$ around the $x$-axis and an angle $\theta$ around the $y$-axis, as shown in Figure 5.5. For convenience and without loss of generality, we assume that there is no fixed rotation around the $z$-axis between $\Psi_{D}$ and $\Psi_{0}$ and that $o_{\Psi_{D}}$ and $o_{\Psi_{m}}$ are not translated with respect to each other. If such rotation and transformations do apply, they can easily be included in the model by multiplying the corresponding $H$-matrices with the current $H_{0}^{m}$.

The transformation matrix $H_{0}^{m}$ describes the transformation from $\Psi_{0}$ to $\Psi_{m}$. The parameters used in $H_{0}^{m}$ are shown in Figure 5.5. $H_{0}^{m}$ consists of a translation from $o_{\Psi_{0}}$ to $o_{\Psi_{m}}$, rotations by the two fixed angles $\vartheta$ and $\theta$ (as described by Bos (1986)) and the dynamic rotation, $\varphi(t)$, around the $z$-axis (a joint in the $z$ direction) due to the vibrations of the payload.

$$
\begin{aligned}
H_{0}^{m} & =H_{D}^{m} \cdot H_{0}^{D} \\
H_{0}^{D} & =\left[\begin{array}{cccc}
1 & 0 & 0 & x_{a} \\
0 & 1 & 0 & y_{a} \\
0 & 0 & 1 & z_{a} \\
0 & 0 & 0 & 1
\end{array}\right] \\
H_{D}^{m} & =H_{D}^{m}(\varphi(t)) \cdot H_{D}^{m}(\theta) \cdot H_{D}^{m}(\vartheta) \\
H_{D}^{m}(\vartheta) & =\left[\begin{array}{cccc}
1 & 0 & 0 & 0 \\
0 & \cos (\vartheta) & -\sin (\vartheta) & 0 \\
0 & \sin (\vartheta) & \cos (\vartheta) & 0 \\
0 & 0 & 0 & 1
\end{array}\right] \\
H_{D}^{m}(\theta) & =\left[\begin{array}{cccc}
\cos (\theta) & 0 & \sin (\theta) & 0 \\
0 & 1 & 0 & 0 \\
-\sin (\theta) & 0 & \cos (\theta) & 0 \\
0 & 0 & 0 & 1
\end{array}\right] \\
H_{D}^{m}(\varphi(t)) & =\left[\begin{array}{cccc}
\cos (\varphi(t)) & 0 & -\sin (\varphi(t)) & 0 \\
\sin (\varphi(t)) & 0 & \cos (\varphi(t)) & 0 \\
0 & 0 & 1 & 0 \\
0 & 0 & 0 & 1
\end{array}\right]
\end{aligned}
$$

Using $H_{0}^{m}$, the force on the payload, expressed in $\Psi_{0}, W^{0}$, can be transformed to $\Psi_{m}$ according to (5.11), in order to find the resulting torque around the modal axis. Let's define $p_{a}^{m}$ as the vector from the modal axis to the linear motor, expressed in the coordinates of $\Psi_{m}$ as shown in Figure 5.4:

$$
p_{a}^{m}=\left[\begin{array}{lll}
x_{a} & y_{a} & z_{a}
\end{array}\right]^{T}
$$

where $z_{a}$ is irrelevant when the rotation around the $z$-axis is considered. $\tau_{z}^{a}$ and the transformation ratio from the actuator to the angular velocity of the first 
vibration mode, $a_{F}^{-1}$-which in fact stems from the mode-shape vector- are:

$$
\begin{aligned}
& W^{0}=\left[\begin{array}{llllll}
0 & 0 & 0 & F_{x}^{0} & 0 & 0
\end{array}\right] \\
& \left(W^{m}\right)^{T}=A d_{H_{m}^{0}}^{T}\left(W^{0}\right)^{T} \\
& =\left[\begin{array}{ll}
\left(\tau^{m}\right)^{T} & \left(F_{x}^{m}\right)^{T}
\end{array}\right]^{T}
\end{aligned}
$$

Assuming the end-effector vibrations to be small motions around an equilibrium, the torque around the modal axis resulting from the linear actuation, $\tau_{z}^{a}$, can be determined by:

$$
\begin{aligned}
\tau^{a} & =p_{a}^{m} \times F_{x}^{m}=\tilde{p}_{a}^{m} F_{x}^{m} \\
& =\left[\begin{array}{ll}
-y_{a} \sin (\theta) & z_{a} \cos (\theta)+x_{a} \sin (\theta)-y_{a} \cos (\theta)
\end{array}\right]^{T} F_{x}^{0} \\
& \Rightarrow \tau_{z}^{a}=\underbrace{\left(-y_{a} \cos (\theta)\right)}_{a_{F}} F_{x}^{0}
\end{aligned}
$$

The twist of a body w.r.t. a certain frame is the same for any arbitrary point on the rigid body. Hence:

$$
T_{e}^{m, 0}=T_{*}^{m, 0}
$$

The linear velocity of a certain point on the rigid body $i$, expressed in $\Psi_{0}$ (fixed world), can be determined using (5.201).

$$
\begin{aligned}
{\left[\begin{array}{c}
\dot{p}_{*}^{0} \\
0
\end{array}\right] } & =\dot{H}_{i}^{0}\left[\begin{array}{ll}
p_{*}^{i} & 1
\end{array}\right]^{T} \\
& =H_{i}^{0} \tilde{T}_{i}^{i, 0}\left[\begin{array}{cc}
p_{*}^{i} & 1
\end{array}\right]^{T} \\
& =\left[\begin{array}{cc}
R_{i}^{0} & p_{i}^{0} \\
0 & 1
\end{array}\right]\left[\begin{array}{cc}
\tilde{\omega}_{i}^{i, 0} & v_{i}^{i, 0} \\
0 & 0
\end{array}\right]\left[\begin{array}{c}
p_{*}^{i} \\
1
\end{array}\right]
\end{aligned}
$$

So for the linear velocity of $P_{e}$ expressed in $\Psi_{0}$, the following holds:

$$
\begin{aligned}
p_{e}^{m} & =\left[\begin{array}{lll}
x_{e} & y_{e} & z_{e}
\end{array}\right]^{T} \\
\dot{p}_{e}^{0} & =\underbrace{\left[\begin{array}{ll}
-R_{m}^{0} \tilde{p}_{e}^{m} & R_{m}^{0}
\end{array}\right]}_{A_{s}} T_{m}^{m, 0}
\end{aligned}
$$

Assuming small $\varphi(t)$ :

$$
\begin{aligned}
& \sin (\varphi(t))=0 \\
& \cos (\varphi(t))=1
\end{aligned}
$$

the elements of matrix $A_{s}(\mathbf{5 . 2 2})$ are ${ }^{\square}$ :

$$
A_{s}=\left[\begin{array}{llll}
A_{s, \omega_{x}} & A_{s, \omega_{y}} & A_{s, \omega_{z}} & A_{s, v}
\end{array}\right]
$$

\footnotetext{
${ }^{2}$ The elements of $A_{s}$ including the term $\varphi(t)$ have not been shown because of their length.
} 


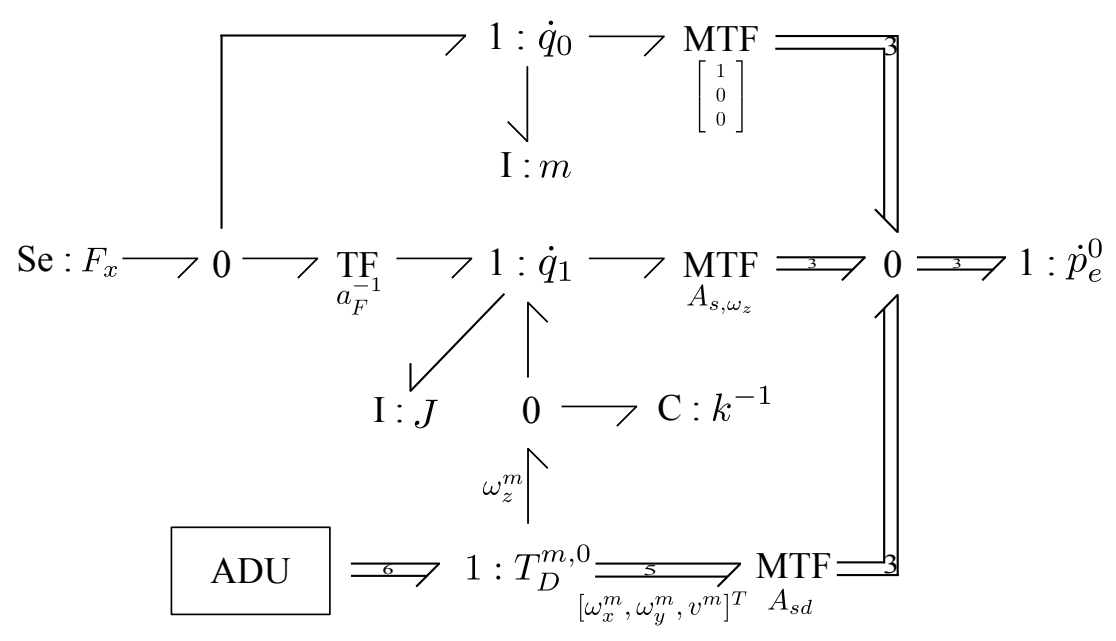

Figure 5.6: Model of the plant including the interaction between the ADU and the end effector. The resulting dynamics from off-axis active damping in the degrees of freedom other than the modal axis are imposed by the ADU actuator(s). Hence, modeling the inertias of the payload around these axes is redundant.

$$
\begin{aligned}
& A_{s, \omega_{x}}=\left[\begin{array}{c}
-y_{e} \sin (\theta) \\
y_{e} \cos (\theta) \sin (\vartheta)-z_{e} \cos (\vartheta) \\
y_{e} \cos (\theta) \cos (\vartheta)+z_{e} \sin (\vartheta)
\end{array}\right] \\
& A_{s, \omega_{y}}=\left[\begin{array}{c}
z_{e} \cos (\theta)+x_{e} \sin (\theta) \\
-x_{e} \cos (\theta) \sin (\vartheta)+z_{e} \sin (\theta) \sin (\vartheta) \\
z_{e} \sin (\theta) \cos (\vartheta)-x_{e} \cos (\theta) \cos (\vartheta)
\end{array}\right] \\
& A_{s, \omega_{z}}=\left[\begin{array}{c}
-y_{e} \cos (\theta) \\
x_{e} \cos (\vartheta)-y_{e} \sin (\theta) \sin (\vartheta) \\
-x_{e} \sin (\vartheta)-y_{e} \sin (\theta) \cos (\vartheta)
\end{array}\right] \\
& A_{s, v}=\left[\begin{array}{ccc}
\cos (\theta) & 0 & -\sin (\theta) \\
\sin (\theta) \sin (\vartheta) & \cos (\vartheta) & \cos (\theta) \sin (\vartheta) \\
\sin (\theta) \cos (\vartheta) & -\sin (\vartheta) & \cos (\theta) \cos (\vartheta)
\end{array}\right]
\end{aligned}
$$

For reference, the model of the plant including the ADU, shown in Figure 5.6 can be used, where:

$$
A_{s d}=\left[\begin{array}{lll}
A_{s, \omega_{x}} & A_{s, \omega_{y}} & A_{s, v}
\end{array}\right]
$$




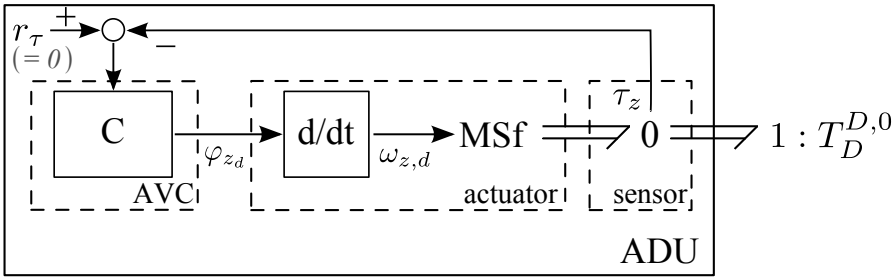

Figure 5.7: Schematic overview of the ADU.

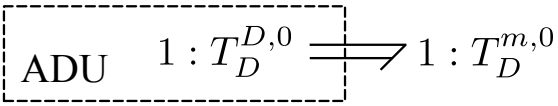

Figure 5.8: The twist relations for modal $A V C ; \Psi_{D}=\Psi_{m}$.

\subsection{Active damping}

To suppress the performance limiting vibrations of the payload, active damping can be applied. This can be done by implementing an active vibration controller. In this section, the AVC and its effect on the plant dynamics are described.

The computations below are done assuming that the ADU is rigid in all directions, but in the direction in which it should apply active damping (in this case around the $z$-axis). However, this is practically impossible to realize. The influence of the compliances of the ADU should be reduced to values much lower than the compliance in the plant causing the vibrations. In addition, both $\vartheta$ and $\theta$ should be relatively small to prevent overloading the ADU in directions other than its own degree of freedom.

\subsubsection{Active vibration controller}

For modal AVC, the Integral Force Feedback (IFF) (5.30) algorithm can be used, which behaves like a viscous damper, $d_{A V C}=K_{I F F}^{-1}$ (Preumont \& Achkire 1997).

$$
C(s)=K_{I F F} / s
$$

The resulting twist due to the actuator(s) of the AVC (see Figure 5.7 ) is given by $(5.3 \mathrm{~J})$, which is a rotation around what is assumed to be the modal axis of the plant (the $z$-axis of $\Psi_{D}$ ).

$$
T_{D}^{D, 0}=\left[\begin{array}{llllll}
0 & 0 & \omega_{z_{d}}^{D, 0} & 0 & 0 & 0
\end{array}\right]^{T}
$$




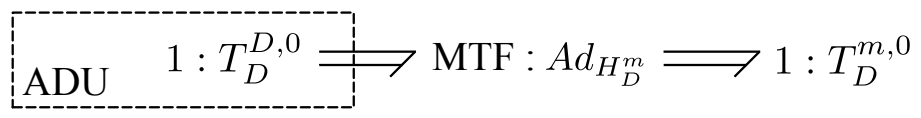

Figure 5.9: The twist relations for off-axis modal $A V C ; \Psi_{D} \neq \Psi_{m}$.
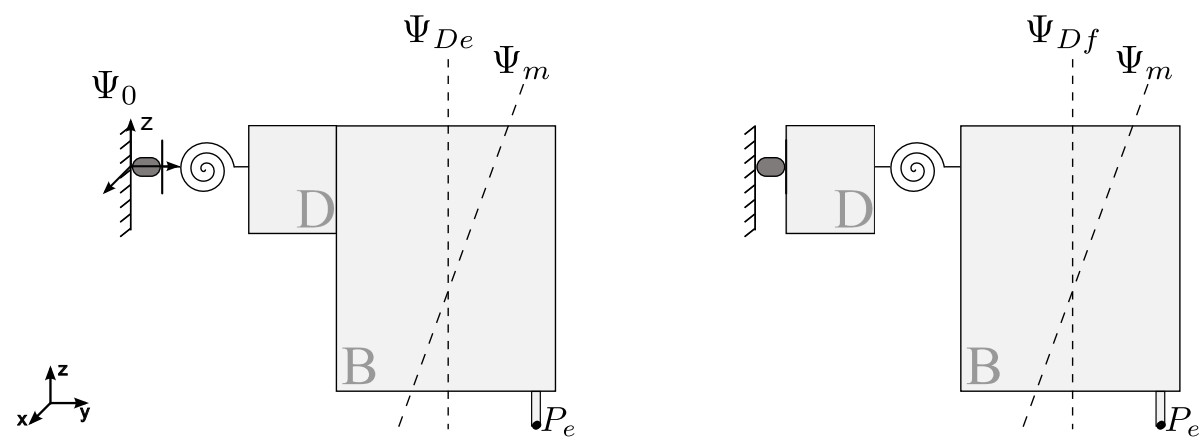

Figure 5.10: Schematic representation of the plant (B) in combination with the $A D U$; on the left side the ADU is fixed to the end effector and operates around $\Psi_{D e}$, on the right side the ADU is fixed to the fixed world and operates around $\Psi_{D f}$. The $z$-axes of $\Psi_{D e}$ and $\Psi_{0}$ are only parallel to each other in absence of vibrations. The $z$-axes of $\Psi_{D f}$ and $\Psi_{0}$ remain parallel at all time.

\subsubsection{ADU and end effector interaction}

In case of modal damping, the ADU operates around the same axis as the modal axis of the plant, so $T_{D}^{D, 0}=T_{D}^{m, 0}$ (see Figure 5.8). However, when the two axes are rotated w.r.t. each other, $T_{D}^{D, 0}$ does not only affect the rotation around the modal axis. This effect is modeled by including a transformation between $T_{D}^{D, 0}$ and $T_{D}^{m, 0}$ as shown in Figure 5.9.

The collocated actuator(s) and sensor(s) for the AVC, which measure the torque and actuate a rotation around the $z$-axis of $\Psi_{D}$, are embedded in an ADU and applied to the plant. The ADU can be fixed to the end effector, which is assumed to be rigid (shown on the left side of Figure 5.10), or fixed in a way that a compliant body is in between the ADU and the end effector (shown on the right side of Figure 5.101). This compliant body can for instance be the guideway causing the rotational vibration mode.

\section{ADU between the physical compliance and the payload}

When mounted on the end effector, the ADU becomes an integrated part of the payload. As a whole, the inertia of the payload is dominant (the ADU is generally smaller) and the guideway is unchanged. The combination of the ADU and the 
end effector thus rotates around practically the same axis as the end effector without an ADU. The operational axis of the ADU (the $z$-axis of $\Psi_{D e}$ ) remains fixed with respect to $\Psi_{m}$, as shown in Figure 5.10 and Figure 5.T]. The activedamping axis is hence different that what is assumed in (Babakhani, de Vries \& van Amerongen 2012C). This difference changes the outcome of the analysis that follows, from the results in (Babakhani, de Vries \& van Amerongen 2012C). The $z$-axes of $\Psi_{D e}$ and $\Psi_{0}$ are only parallel to each other when the payload does not vibrate.

$$
\begin{gathered}
H_{D e}^{m}=H_{D}^{m}(\theta) \cdot H_{D}^{m}(\vartheta) \\
T_{D e}^{m, 0}=A d_{H_{D e}^{m}} T_{D}^{D, 0} \\
=\left[\begin{array}{c}
\sin (\theta) \cos (\vartheta) \\
-\sin (\vartheta) \\
\cos (\theta) \cos (\vartheta) \\
0 \\
0 \\
0
\end{array}\right] \omega_{z_{d}}^{D, 0}
\end{gathered}
$$

\section{ADU between the fixed world and the physical compliance}

When fitted between the fixed world and the physical compliance, the ADU axis (the $z$-axis of $\Psi_{D f}$ ) is, and remains parallel to the $z$-axis of the fixed world (The $z$-axes of $\Psi_{D f}$ and $\Psi_{0}$ are parallel).

$$
H_{D f}^{m}=H_{D}^{m}(\varphi(t)) \cdot H_{D}^{m}(\theta) \cdot H_{D}^{m}(\vartheta)
$$
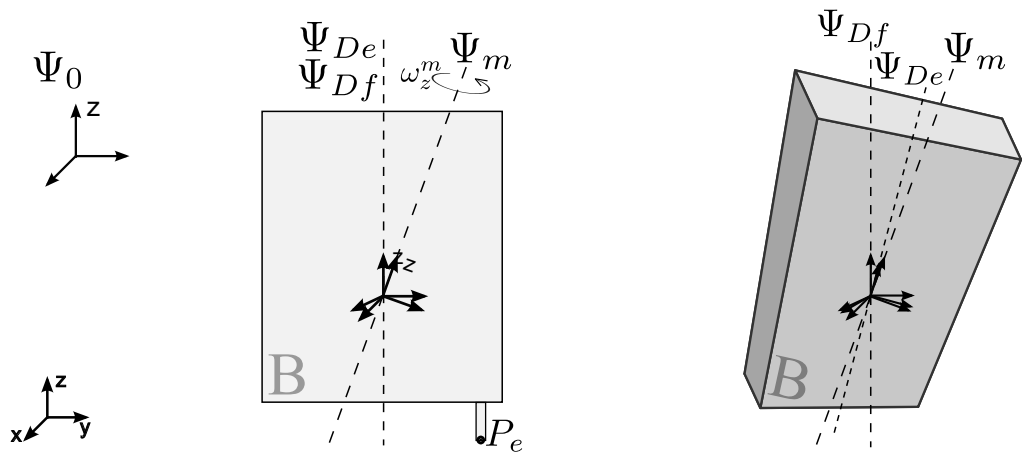

Figure 5.11: Schematic representation of the plant (B), its coordinate frame $\Psi_{m}$ and the active damping frames belonging to the $A D U$, fixed to the fixed world $\Psi_{D f}$ or to the end effector $\Psi_{D e}$. The ADU frames are rotated by the angles $\vartheta$ and $\theta$. When $B$ rotates around its modal axis, $\Psi_{D f}$ remains fixed w.r.t. $\Psi_{0}$ while $\Psi_{D e}$ remains fixed w.r.t. $\Psi_{m}$. 


$$
\begin{aligned}
& T_{D f}^{m, 0}=A d_{H_{D f}^{m}} T_{D}^{D, 0} \\
& =\left[\begin{array}{c}
\sin (\varphi(t)) \sin (\vartheta)+\cos (\varphi(t)) \sin (\theta) \cos (\vartheta) \\
-\cos (\varphi(t)) \sin (\vartheta)-\sin (\varphi(t)) \sin (\theta) \cos (\vartheta) \\
\cos (\theta) \cos (\vartheta) \\
0 \\
0 \\
0
\end{array}\right] \omega_{z_{d}, 0}^{D, 0}
\end{aligned}
$$

Assuming small $\varphi(t), H_{D e}^{m} \approx H_{D f}^{m}$. This means that there is not a significant difference between the two different assembly locations for the ADU. It should be noted that other factors, such as tilt between the ADU and the end effector, might still make it preferable to mount the ADU as close as possible to the performance metric. These factors however are not investigated here.

\section{The effect on modal damping}

Due to the transformation on $T_{D}^{D, 0}$, stemming from the misalignment of the assumed and the real axis of vibration, the rotation imposed by the AVC, $\omega_{z_{d}}^{D, 0}$, results in a factor $\cos (\vartheta) \cos (\theta)$ smaller rotation of the payload. The open-loop transfer function of the AVC loop is given by:

$$
H_{o l}(s)=\frac{\tau_{z}}{\omega_{z_{d}}}=\frac{\cos ^{2}(\vartheta) \cos ^{2}(\theta) k s}{s^{2}+\omega_{e, 1}^{2}}
$$

This results in the closed-loop transfer function:

$$
H_{c l}(s)=\frac{\tau_{z}}{r_{\tau}}=\frac{\cos ^{2}(\vartheta) \cos ^{2}(\theta) K_{I F F} k s}{s^{2}+\cos ^{2}(\vartheta) \cos ^{2}(\theta) K_{I F F} k s+\omega_{e, 1}^{2}}
$$

thus a damping term of:

$$
d_{m, 1}=\cos ^{2}(\vartheta) \cos ^{2}(\theta) K_{I F F} k
$$

So, the smaller the deviation between the modal axis and the active damping axis (small $\theta$ and $\vartheta$ ), the higher the achieved modal damping, as can be expected. In addition, the actuation by the AVC induces rotations around both the $x$ and the $y$ axes of $\Psi_{m}$, which is in general undesired.

For comparison, when the active-damping axis and the modal axis are aligned, $\theta=\vartheta=0$, the damping injection by the AVC is optimal, and more importantly, the ADU does not cause any undesired rotations around the $x$ and the $y$ axes. This is also evident from the closed-loop transfer function (5.40).

$$
H_{o l}(s)=\frac{\tau_{z}}{\omega_{z_{d}}}=\frac{k s}{s^{2}+\omega_{e, 1}^{2}}
$$




$$
H_{c l}(s)=\frac{\tau_{z}}{r_{\tau}}=\frac{K_{I F F} k s}{s^{2}+K_{I F F} k s+\omega_{e, 1}^{2}}
$$

Hence, the resulting modal damping is:

$$
d_{m, 1}=K_{I F F} k
$$

Equation (5.38) implies that if $\vartheta$ and $\theta$ have constant values, the damping term of the off-axis AVC (5.38) can be increased to the value of (5.41) by increasing $K_{I F F}$. However, the decrease of the achieved damping in case of off-axis vibration control is in practice inevitable due to the resulting decreased controllability of the vibration mode. This can be shown by including $k_{p l}$ (see Section 3.3.T). The open-loop transfer function of the AVC loop becomes:

$$
H_{o l}(s)=\frac{\tau_{z}}{\omega_{z_{d}}}=\left(k_{p l}+\cos ^{2}(\vartheta) \cos ^{2}(\theta) k\right) \frac{s^{2}+\frac{k_{p l} k}{J\left(k_{p l}+\cos ^{2}(\vartheta) \cos ^{2}(\theta) k\right)}}{s^{2}+\omega_{e, 1}^{2}}
$$

So, the antiresonance frequency is equal to:

$$
\omega_{a, 1}=\frac{k_{p l} k}{J\left(k_{p l}+\cos ^{2}(\vartheta) \cos ^{2}(\theta) k\right)}
$$

The larger the values of $\vartheta$ and $\theta$, the smaller $\cos ^{2}(\vartheta) \cos ^{2}(\theta)$, and hence the higher the antiresonance frequency of the AVC loop (See (B.8) for comparison). An increase of the antiresonance frequency decreases the difference between the resonance and antiresonance frequencies, which decreases the maximum achievable damping (4.T2).

\subsection{Simulations}

The results of the previous section are evaluated here in simulation. Two sets of parameters have been used. One simulation is performed assuming that the modal axis is rotated w.r.t. the $z$-axis of $\Psi_{0}$ (Figure [5.12). The other simulation is performed assuming the modal axis to be parallel to the $z$-axis of $\Psi_{0}$ (Figure [5.T3). In both cases, the active damping axis is assumed to be rotated w.r.t. the modal axis by the angles $\vartheta_{D}$ and $\theta_{D}$. The other parameters of the model used for simulations (see Figure 5.6), are listed in Table 5.T. The off-axis AVC is simulated for both locations of the ADU, as shown in Figure 5.J0. However, the difference is not visible in the plots, as expected.

The dashed gray lines in Figure 5.12 and Figure 5.13 show the linear velocity of the end effector, expressed in $\Psi_{0}$, on application of a force, $F_{x}$, in the $x$-direction in $\Psi_{0}$, without AVC. The oscillatory behavior due to the rotational vibration mode is clearly visible, especially in the $x$-direction. 


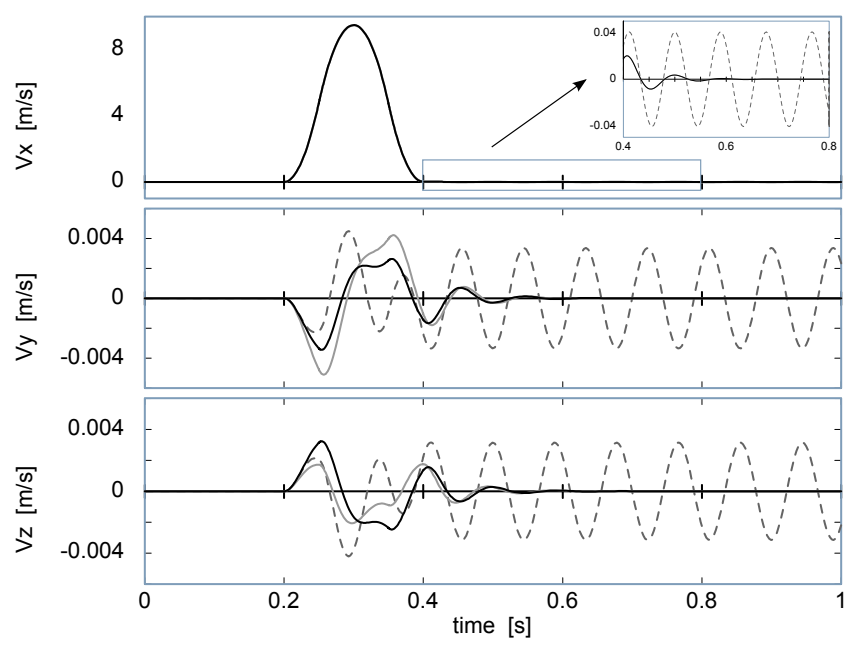

Figure 5.12: $\dot{p}_{e}^{0}(\mathrm{~m} / \mathrm{s})$ of the plant v.s. time $(\mathrm{s})$ in $x(V x), y(V y)$, and $z(V z)$ direction. $\vartheta=-7^{\circ}$ and $\theta=5^{\circ}$; gray dashed line: without AVC, black: modal AVC with $\Psi_{D}=\Psi_{m}$, gray solid line: off-axis AVC.

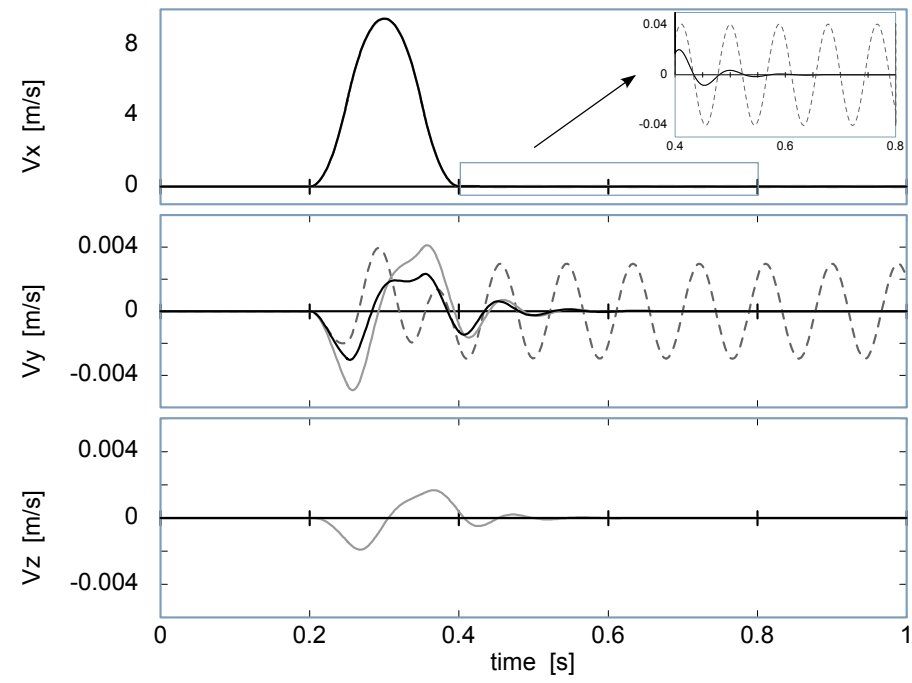

Figure 5.13: $\dot{p}_{e}^{0}(\mathrm{~m} / \mathrm{s})$ of the plant v.s. time $(\mathrm{s})$ in $x(V x), y(V y)$, and $z(V z)$ direction. $\vartheta=\theta=0$; gray dashed line: without AVC, black line: modal AVC with $\Psi_{D}=$ $\Psi_{m}$, gray solid line: off-axis AVC. 
Table 5.1: Parameters of the model used for simulations.

\begin{tabular}{|c|cc|}
\hline \hline Parameter & Value & Unit \\
\hline$m$ & 1.5 & $\mathrm{~kg}$ \\
$k$ & $2.5 e 3$ & $\mathrm{Nm} / \mathrm{rad}$ \\
$J$ & 0.5 & $\mathrm{kgm}^{2} / \mathrm{rad}$ \\
\hline$\theta_{D}$ & 4 & $\circ$ \\
$\vartheta_{D}$ & -3 & $\circ$ \\
$K_{I F F}$ & 0.015 & \\
\hline$x_{e}$ & 0.8 & $\mathrm{~cm}$ \\
$y_{e}$ & 11 & $\mathrm{~cm}$ \\
$z_{e}$ & -6 & $\mathrm{~cm}$ \\
\hline$x_{a}$ & 0.5 & $\mathrm{~cm}$ \\
$y_{a}$ & 5.5 & $\mathrm{~cm}$ \\
\hline \hline
\end{tabular}

The black solid lines show the dynamics of the end effector after applying AVC around the same axis as the vibrations axis; $\Psi_{D}=\Psi_{m}$. The plots show that AVC improves the dynamics of the end effector considerably.

The solid gray lines show the effect of off-axis AVC. Both simulations show that the dynamics of the end effector have changed compared to when $\Psi_{D}=$ $\Psi_{m}$ (black line). Although the performance is improved compared to the undamped plant dynamics (dashed lines), there is a clear difference between the performance of the off-axis AVC and the ideal situation (black line). In terms of achieved damping, the difference is barely visible.

Figure 5.12 shows a situation in which the performance in the $y$-direction is worsened, whereas the performance in the $z$-direction is improved as a result of the deviation between the damping axis and the modal axis. Figure 5.13 shows a configuration for which the performance in both $y$-direction and $z$-direction worsen. Figure 5.13 shows that the off-axis AVC introduces dynamics in the $z$ direction that were not present without off-axis AVC.

\subsection{Conclusions}

In this chapter, the interaction between the active damping unit and a plant suffering from rotational vibrations was given in a 3D setting. This interaction was modeled using the principles of both modal modeling and the screw theory. Using the transformation matrices, it was shown that the location of the active damping unit with respect to the physical compliance in the plant does not have a significant effect on the achieved active damping. The analysis of off-axis 
active vibration control showed that the achievable damping decreases due to the deviation of the damping axis from the vibration axis. This decrease is however not significant for small angles. Off-axis AVC induces dynamics that depending on the specific configuration of the plant, could worsen the performance. The results were verified be means of simulation. 


\section{Chapter 6}

\section{Loss of Collocation}

Abstract - Adding active damping in a collocated fashion to a badly damped mechanical plant guarantees the stability of the closed-loop system. However, collocation is lost beyond a certain frequency due to restrictions imposed by practical implementation. In this chapter, the effect of the first noncollocated vibration mode in the active damping loop is analyzed on the basis of its most relevant parameters. The results are summarized in two figures that provide guidelines for both designing an active damper unit and tuning its controller.

This chapter is a revised version of (Babakhani, de Vries \& van Amerongen 2012b);

"Design Guidelines concerning the Destabilizing High-frequency Mode of an Integral Force Feedback-based Active Damping Unit"

B. Babakhani and T. J. A. de Vries and J. van Amerongen

Proceedings of the IEEE/ASME International Conference on Advanced Intelligent Mechatronics, Taiwan, July 11-14, 2012. 


\subsection{Introduction}

Industrial machines generally suffer from loss of performance due to badly damped resonances in the mechanical structure. When passive measures are not sufficient or cannot be realized, active methods can be used to add damping to the undesired vibrations. Examples of active damping are active mounts for vibration isolation as implemented by Clark \& Robertshaw (1997), smart discs embedded in lithographic machines by van Schothorst (I1999) and Holterman \& de Vries (2004), and smart structures in large flexible spacecrafts (see Inman 20001). Active damping can also improve the performance of motion systems such as pick-and-place machines (see Verscheure et al. 2006) and robot arms (see Choil et al. (1999).

The implementation of active damping can be done using various control algorithms and sensor- and actuator configurations, an overview of which can be found in (Gawronskil 2004). The sensor- and actuator configurations can be classified in two groups: collocated or noncollocated (Miu 1992, Preumont 1997). In this chapter the collocated group is considered because of the guarantee of stability that is obtained when the control algorithm entails an energetically Intrinsically Passive Controller (IPC) (Stramigioli \& Bruyninckx 200T). Hence, integral force feedback (Preumont \& Achkire 1997), which is an IPC, is chosen here, although model based algorithms may achieve better performance (Ehmann 20102).

However, in practice collocation can only be realized within a limited frequency region; either mechanical or electronic properties of the Active Damper Unit (ADU) result in the loss of collocation beyond a certain frequency. So an important question is where the first noncollocated vibration mode in the active damping loop should be to avoid instability and retain the above mentioned stability guarantee.

In this chapter, the characteristics of the first noncollocated vibration mode are identified that have a significant role in the stability properties of the active damping loop. The effect of each characteristic is explained using a root-locus plot (Franklin et al, 2006) of the active damping loop. The transfer function of the active damping loop is used to obtain stability regions. The stability regions presented in this chapter can be used as guidelines during both the design of the active damping unit and the tuning of the control algorithm to prevent instability.

The model that is used in this chapter is the same model as in Section [5.2, which was derived in Section 2.2., namely:

$$
\frac{x_{k}}{F_{x, l}}(s)=\sum_{i=1}^{n} \frac{\phi_{j i} \phi_{k i}}{m_{m, i} s^{2}+k_{m, i}}
$$




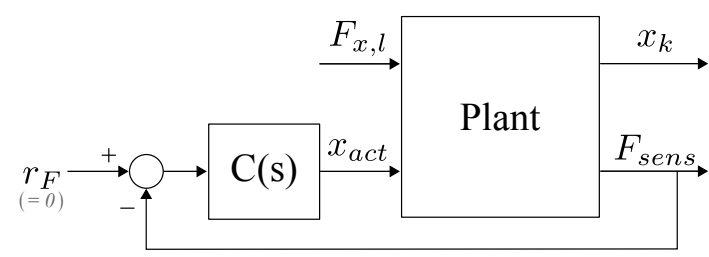

Figure 6.1: The schematic overview of the plant in active damping loop. The plant transfer function from $F_{x, l}$ to $x_{k}$ is given by (6.]), the plant transfer function from $x_{a c t}$ to $F_{\text {sens }}$ is given by (6.70), and the controller is described by (6.3). The reference signal for the controller is $r_{F}=0$.

Where the resonance frequency of the $i$-th vibration mode, $\omega_{e, i}$, is described by:

$$
\omega_{e, i}=\sqrt{\frac{k_{m, i}}{m_{m, i}}}
$$

This chapter is organized as follows. First, collocated active damping is explained in Section 6.2; The control algorithm and its effect on both the collocated and noncollocated vibration modes can be found in this section. Next, the guidelines and rules of thumb concerning the properties of the first noncollocated vibration mode are discussed in Section 6.3. Section 6.4 presents the conclusions.

\subsection{Collocated active damping}

Collocated control focuses on the addition of damping to a dominant vibration mode, called the target mode here. The controller is tuned to add optimal damping to the target mode, but it will also add damping to collocated higherorder modes. Here, a Leaking Integral Force Feedback (LIFF) algorithm is chosen for the active damping controller, for which a collocated force sensor and position actuator are required (see Figure 6.T).

\subsubsection{Leaking integral force feedback}

Leaking integral force feedback (6.3) behaves like a viscous damper, $d=K_{L I F F}^{-1}$, and a static stiffness, $k=p_{L P} \cdot K_{L I F F}^{-1}$ (see Section B.3.T and Preumont \& Achkire (1997)).

$$
C(s)=\frac{K_{L I F F}}{s+p_{L I F F}}
$$


The maximum achievable damping for a single mode (for $\omega_{a}>\omega_{e} / 3$ ) can be determined according to (Preumont 1997):

$$
\xi_{e, i}^{\max }=\frac{\omega_{e, i}-\omega_{a, i}}{2 \omega_{a, i}}
$$

where $\omega_{e, i}$ denotes the resonance frequency of the target mode and $\omega_{a, i}$ its antiresonance frequency. $\xi_{e, i}^{\max }$ is achieved by the following open-loop gain:

$$
K_{o l}^{\xi^{\max }}=\omega_{e, i} \sqrt{\frac{\omega_{e, i}}{\omega_{a, i}}}
$$

The $K_{L I F F}$ that leads to $\xi^{\max }$ is the ratio of $K_{o l}^{\xi^{\max }}$ to the high-frequency gain level of the Frequency Response Function (FRF) from the position actuator to the force sensor.

To improve the static stiffness of the plant and prevent actuator saturation, a high-pass filter (6.6) can be added to the LIFF algorithm.

$$
H_{H P}(s)=\frac{s}{s+p_{H P}}
$$

By doing so, the guarantee of stability is lost in general, but according to Holterman (20122), if the cut-off frequency of the high-pass filter is small w.r.t. the resonance frequency, the closed-loop stability is preserved. Hence, the active damping controller is tuned using the rules of thumb given by Holterman (20012), namely:

$$
\begin{aligned}
& p_{L P} \leq 0.2 \omega_{e, i} \\
& p_{H P} \leq 0.2 p_{L P}
\end{aligned}
$$

\subsubsection{Active damping loop}

To derive the transfer function of the active damping loop, the contribution of each resonance mode of the plant, $\omega_{e, i}\left(6_{2}\right)$, should be determined. For convenience, we assume that the ADU consists of only one actuator and one sensor. The reader is referred to (Holterman 2002) for a detailed derivation of the transfer function of the active damping loop in a general setting.

As mentioned in Section 2.2, each mode can be described by the modal parameters $m_{m, i}$ and $k_{m, i}$. The position actuation by the ADU, $x_{a c t}$, results in a modal contribution termed $q_{a c t, i}$, according to:

$$
\begin{aligned}
x_{a c t} & =\phi_{a i} q_{a c t, i} \\
\frac{q_{i}}{q_{a c t, i}}(s) & =\frac{k_{m, i}}{m_{m, i} s^{2}+k_{m, i}}
\end{aligned}
$$


The contribution of each modal force $F_{m, s e n s, i}$, to the force measured by the ADU sensor $F_{\text {sens }}$, is given by:

$$
\begin{aligned}
F_{m, \text { sens }, i} & =k_{m, i}\left(q_{a c t, i}-q_{i}\right) \\
F_{\text {sens }} & =\sum_{i=1}^{n} \phi_{s i} F_{m, \text { sens }, i}
\end{aligned}
$$

The transfer function of the active damping loop from the position actuator to the force sensor of the ADU is given by the sum of each modal contribution, which results in:

$$
\frac{F_{\text {sens }}}{x_{a c t}}(s)=k_{0}+\sum_{i=1}^{n} \frac{\phi_{a i} \phi_{s i} k_{m, i} s^{2}}{s^{2}+\omega_{e, i}^{2}}
$$

Here, $\phi_{a i}$ and $\phi_{s i}$ are elements of the matrix for transforming the ADU coordinates to modal coordinates and $k_{0}$ represents the low-frequency level of the FRF from position actuator to the force sensor. For collocated active damping $\left(\phi_{a i}=\phi_{s i}\right)$, and in case a piezo actuator and sensor stack is used, $k_{0}$ incorporates the stiffness paralle to the piezo stack, in combination with stack stiffness.

The model of the plant, reduced to having only the target mode, $i=1$, is given by:

$$
\begin{aligned}
P(s) & =\frac{F_{\text {sens }}}{x_{\text {act }}}(s) \\
& =\frac{\left(k_{1}+k_{0}\right) s^{2}+k_{0} \omega_{e, 1}^{2}}{s^{2}+\omega_{e, 1}^{2}} \\
& =\left(k_{1}+k_{0}\right) \cdot \frac{s^{2}+\frac{k_{0}}{k_{1}+k_{0}} \omega_{e, 1}^{2}}{s^{2}+\omega_{e, 1}^{2}}
\end{aligned}
$$

where $k_{1}=\phi_{s 1} \phi_{a 1} k_{m, 1}$ and since collocated, $\phi_{s 1}=\phi_{a 1}$.

Rewriting (6.11) in terms of resonance and antiresonance frequencies, results in the following transfer function:

$$
P(s)=g \cdot \frac{s^{2}+\omega_{a, 1}^{2}}{s^{2}+\omega_{e, 1}^{2}}
$$

where $g$ is the high-frequency gain and $\omega_{a, 1}$ is a function of $k_{0}, k_{1}$ and $\omega_{e, 1}$. The ratio of the resonance frequency to the antiresonance frequency, termed $\eta$ (6.T3), is determined by $k_{0}, k_{m, 1}, \phi_{s 1}$, and $\phi_{a 1}$.

$$
\eta_{1}=\frac{\omega_{e, 1}^{2}}{\omega_{a, 1}^{2}}
$$

Including other modes in the model can be done by just adding its modal contribution to the transfer function according to (6.T0). The plant transfer 
Table 6.1: Mode types.

\begin{tabular}{|c||c|c|c|c|c|}
\hline \hline$\eta$ & $\eta>1$ & $\eta=1$ & $0<\eta<1$ & $\eta=0$ & $\eta<0$ \\
\hline Type & AR & $\mathrm{U}$ & $\mathrm{RA}$ & $\mathrm{R}$ & $\mathrm{N}$ \\
\hline
\end{tabular}

function including one additional vibration mode, $i=2$, becomes after rewriting:

$$
P(s)=g \cdot \frac{s^{2}+\omega_{a, 1}^{2}}{s^{2}+\omega_{e, 1}^{2}} \cdot \frac{s^{2}+\omega_{a, 2}^{2}}{s^{2}+\omega_{e, 2}^{2}}
$$

For the second vibration mode, $k_{2}=\phi_{a 2} \phi_{s 2} k_{m, 2}$ applies (similar to (6.JI)). In case the additional mode is noncollocated, $\phi_{a 2} \neq \phi_{s 2}$.

The antiresonance frequencies of the active damping loop are determined by $k_{0}$, $\phi_{a i} \phi_{s i}, k_{m, i}$ and $\omega_{e, i}$.

\subsubsection{Stability and higher-order modes}

The stability of the active damping loop depends greatly on the type of higherorder modes. The type of a certain mode can be characterized using parameter $\eta$, as can be seen in Table 6.J (see also Section 2.2. and Table 2.TI). Here R stands for resonance, A for antiresonance, $\mathrm{U}$ for unobservable/uncontrollable $(\mathrm{R}=\mathrm{A})$, and $\mathrm{N}$ for non-minimum phase; $\mathrm{AR}$ implies first antiresonance, then resonance (Coelingh et al. 2002). Types $\mathrm{U}$ and $\mathrm{N}$ are no longer considered hereafter.

All modes in the active damping loop have an effect on the location of the antiresonance frequencies, and hence on the maximum achievable damping for the target mode, $\xi_{1}^{\max }$. But as long as the higher-order modes are of the AR type, the stability of the active damping loop is preserved. This special pole zero pattern, which typically occurs for collocated control, results in poles remaining in the left half of the $s$-plane for an increasing gain of the active damping controller (see Figure 6.2a). However, if modal damping is small, modes of the RA and R type are destabilized beyond a certain $K_{L I F F}$, as shown in Figure $6.2 b^{\mathbb{m}}$. In this chapter, the effect of these two types of noncollocated modes is considered. From now on, the phrase "noncollocated mode" refers to a higher-order mode of either RA or R type.

The influence of a noncollocated mode on the stability of the active damping loop can be analyzed by means of three characteristics, namely:

- $\xi_{e, i}$ : the relative damping of a mode

\footnotetext{
${ }^{1}$ The root-locus plots in this chapter are only depicted for the positive imaginary axis
} 


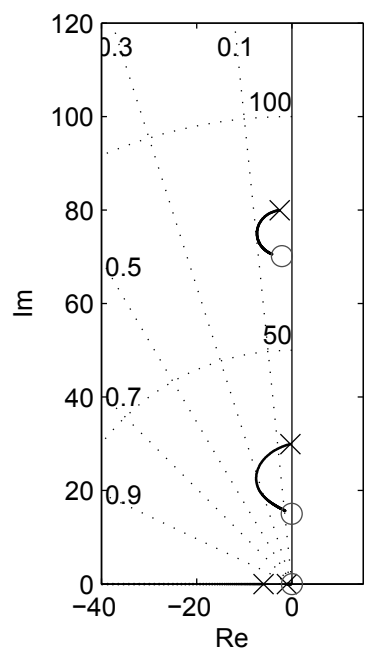

(a) $\eta_{2}<1$

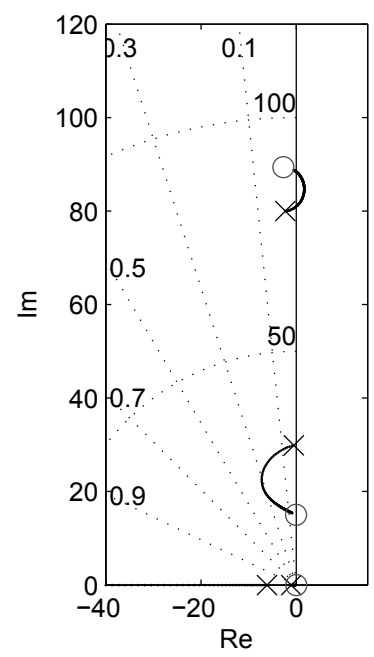

(b) $\eta_{2}>1$

Figure 6.2: Root-locus plot of the active damping loop, including two modes, for an increasing $K_{L I F F}$. Left: Type $A R$, right: type $R A$.

- $\eta_{i}$ : the relative distance between resonance and antiresonance of a mode

- $\omega_{e, i+1} / \omega_{e, i}$ : the relative distance between resonance of the target mode and the higher-order mode

Using relative characteristics rather than the parameters of the stated problem themselves, omits dimensional aspects from the analysis.

Incorporating $\xi_{e, i}$ in (6.14) results in (6.15) that can be used to investigate the effect of relative damping on the dynamics of the active damping loop.

$$
\begin{aligned}
P(s) & =g \cdot P_{1}(s) \cdot P_{2}(s) \\
P_{1}(s) & =\frac{s^{2}+2 \xi_{a, 1} \omega_{a, 1} s+\omega_{a, 1}^{2}}{s^{2}+2 \xi_{e, 1} \omega_{e, 1} s+\omega_{e, 1}^{2}} \\
P_{2}(s) & =\frac{s^{2}+2 \xi_{a, 2} \omega_{a, 2} s+\omega_{a, 2}^{2}}{s^{2}+2 \xi_{e, 2} \omega_{a, 2} s+\omega_{e, 2}^{2}}
\end{aligned}
$$

The root-locus plots in Figure $\sqrt{6.3}$ illustrate the general effect of the listed characteristics. Each time, the parameter that has been varied is indicated by 


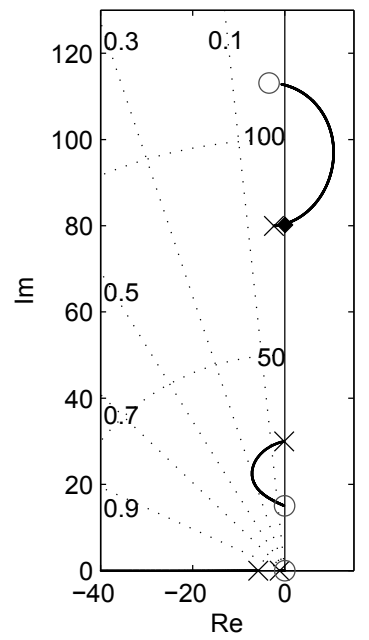

(a) $\xi_{e, 2}=0.03, \eta_{2}=0.5$, $\omega_{e, 2} / \omega_{e, 1}=2.7, \downarrow: \varrho=0.26$

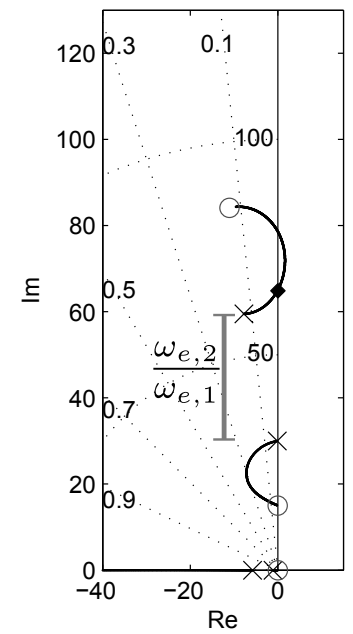

(c) $\xi_{e, 2}=0.13, \eta_{2}=0.5$, $\omega_{e, 2} / \omega_{e, 1}=2, \downarrow: \varrho=1.12$

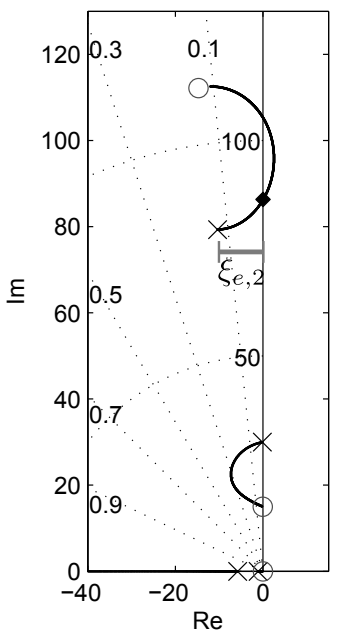

(b) $\xi_{e, 2}=0.13, \eta_{2}=0.5$, $\omega_{e, 2} / \omega_{e, 1}=2.7, \bullet: \varrho=1.58$

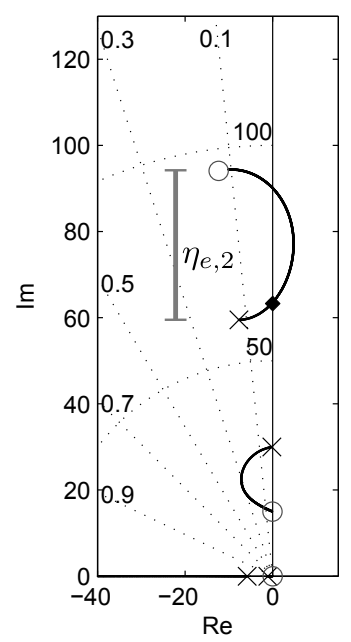

(d) $\xi_{e, 2}=0.13, \eta_{2}=0.4$, $\omega_{e, 2} / \omega_{e, 1}=2, \downarrow: \varrho=0.78$

Figure 6.3: The role of the three characteristics of the noncollocated mode, $\xi_{e, 2}, \eta_{2}$ and $\omega_{e, 2} / \omega_{e, 1}$ on the stability of the active damping loop. 
a gray line in the plot. The black lozenge indicates the border of instability. The corresponding active damping gain, expressed as a fraction of the optimal gain $\varrho\left(\right.$ (6.16) , also referred to as $\varrho_{c c}$, is included in the captions.

$$
\varrho=\frac{K_{L I F F}}{K_{L I F F}^{\xi^{m a x}}}
$$

$\varrho$ is a measure of the achieved active damping; $\varrho \approx 0$ implies that hardly any active damping is added and $\varrho_{c c}>1$ means that maximum achievable damping for the target mode, $\xi_{e, 1}^{\max }$, is feasible and thus the corresponding $K_{L I F F}$ does not destabilize the higher-order noncollocated mode.

Looking at Figure 6.3, the following observations hold:

- more relative damping in the noncollocated mode, i.e. a higher $\xi_{e, 2}$, moves the root locus towards the left half-plane. Hence this increases $\varrho_{c c}$ (compare Figure 6.3a with Figure 6.3b)

- a larger resonance of the noncollocated mode, i.e. increasing $\omega_{e, 2} / \omega_{e, 1}$, slows the movement of the higher-order pole, resulting in an increase of $\varrho_{c c}$ (compare Figure 6.3b with Figure 6.3d)

- an increase in distance between resonance and antiresonance, i.e. a larger $\eta_{2}$, changes the curvature of the root-locus belonging to the noncollocated mode, leading to a lower $\varrho_{c c}$ (compare Figure 6.30 with Figure 6.3d).

Of course, the characteristics of the collocated modes also influence the locus of the noncollocated mode. The number of collocated modes and their characteristics are in general unknown. Nevertheless, their effect can be illustrated using the target mode.

The parameter $\xi_{e, 1}^{\max }$ (6.4) can be used to characterize the target mode. $\xi_{e, 1}^{\max }$ is the damping of the target mode, as a result of active damping. In addition, it incorporates the effect of $\eta_{1}$, since the value of $\xi_{e, 1}^{\max }$ depends on the location of $\omega_{e, 1}$ w.r.t. $\omega_{a, 1}$.

Figure 6.4 shows the effect of the target mode on the stability of the noncollocated mode. Comparing Figure 6.4a with Figure 6.4b (and also with Figure 6.3d) it can be concluded that a higher $\xi_{e, 1}^{\max }$ increases $\varrho_{c c}$. Note that these examples should be interpreted differently from Figure 6.3. A higher $\varrho_{c c}$ by itself is not necessarily an improvement; it merely shows the proportion of $\xi_{e, 1}^{\max }$ that is feasible, keeping in mind that the value of $\xi_{e, 1}^{\max }$ is different for Figure 6.4a and Figure $6.4 \mathrm{~b}$. Hence, this example only shows how $\xi_{e, 1}^{\max }$ affects the locus of the noncollocated mode. 


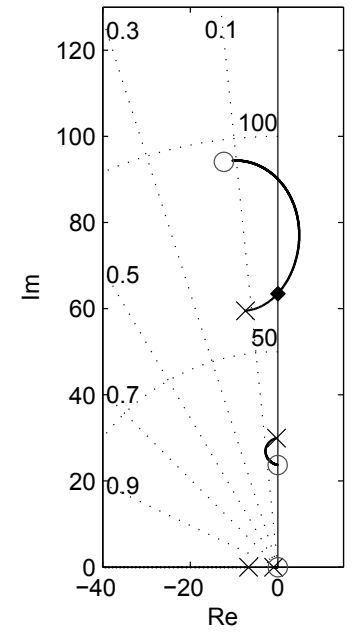

(a) $\xi_{e, 1}^{\max }=0.1, \bullet: \varrho=0.75$

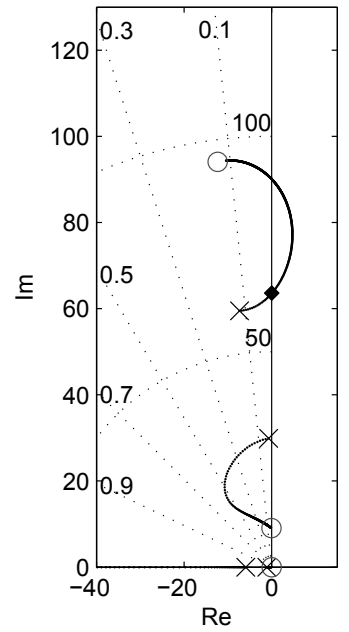

(b) $\xi_{e, 1}^{\max }=0.5, \diamond: \varrho=0.85$

Figure 6.4: The effect of the target mode, $\xi_{e, 1}^{\max }$ on the locus of the noncollocated mode, $\xi_{e, 2}=0.13, \eta_{2}=0.4, \omega_{e, 2} / \omega_{e, 1}=2$ (compare with Figure 6.3d).

\subsection{Stability analysis}

There are two ways of approaching the stability analysis, namely from the perspective of designing an active damper or from the perspective of tuning the active damping controller. The former assumes freedom in designing an ADU while the latter rather copes with the limitations of the ADU.

For the stability analysis (6.15) is used as the plant transfer function. The target mode is represented by $i=1$ for which $\eta_{1}>1$, and the first noncollocated mode is represented by $i=2$ and $0 \leq \eta_{2}<1$.

\subsubsection{Active damper design}

When designing the ADU, the key question is what the lower bound is for the frequency of the first noncollocated mode for the active damping loop to remain stable when the active damping controller is tuned to obtain the maximum achievable active damping.

The lines in Figure 6.5 and Figure 6.6 show the minimum required $\omega_{e, 2} / \omega_{e, 1}$ to avoid instability for various values of $\xi_{e, 2}$. Figure $\measuredangle 5$ also shows the effect of $\xi_{e, 1}^{\max }$ on the minimum required $\omega_{e, 2} / \omega_{e, 1}$. This figure shows that a larger $\xi_{e, 1}^{\max }$ imposes 


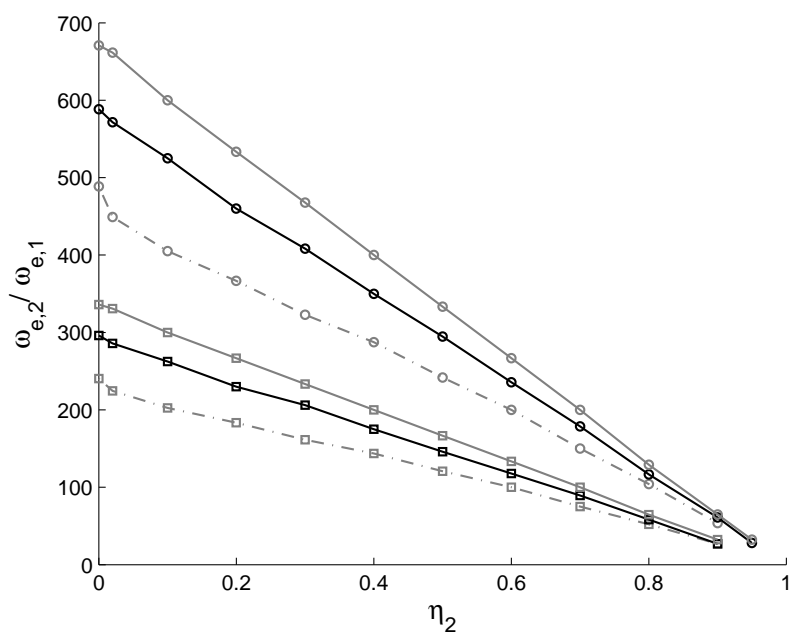

Figure 6.5: $A D U$ design plot. Minimum required frequency ratios for various $\xi_{e, 2}$ and $\xi_{e, 1}^{\max } . \circ: \xi_{e, 2}=0.001$ and $\square: \xi_{e, 2}=0.002$. Gray solid line: $\xi_{e, 1}^{\max }=0.5$, Black solid line: $\xi_{e, 1}^{\max }=0.3$, Gray dash-dotted line: $\xi_{e, 1}^{\max }=0.1$.

a higher $\omega_{e, 2} / \omega_{e, 1}$. The beneficial effect of relative damping in the noncollocated mode becomes very clear from both Figure 5.5 and Figure 5.6. However, it is difficult to design for damping in practice, which is the reason for the need to apply active damping in the first place. Also, it shows that we should strive for a design in which the antiresonances of the noncollocated modes remain as close to the resonances as possible, especially in case of low relative damping.

Furthermore, these figures can be used to deduce some rules of thumb. As shown in Figure 6.5, the stability bounds depend on $\xi_{e, 1}^{\max }$. Although it might be possible to achieve relatively high $\xi_{e, 1}^{\max }$, it is generally both difficult and undesirable, since it decreases the static stiffness of the plant ${ }^{\mathbb{D}}$. This is of course a design consideration. But assuming that $\xi_{e, 1}^{\max }>0.5$ is undesirable (if not unachievable), a linear formula for the minimum required $\omega_{e, 2} / \omega_{e, 1}$ as a function of both $\eta_{2}$ and $\xi_{e, 2}$ can be deduced from the stability bounds, namely:

$$
\left(\frac{\omega_{e, 2}}{\omega_{e, 1}}\right)_{\text {min }}=0.7 \cdot \xi_{e_{2}}^{-1}\left(1-\eta_{2}\right)
$$

\footnotetext{
${ }^{2}$ In addition to an increase in the absolute value of the real part of the resonance poles (higher damping), a decrease of the absolute value of their imaginary part occurs as a result of active damping
} 


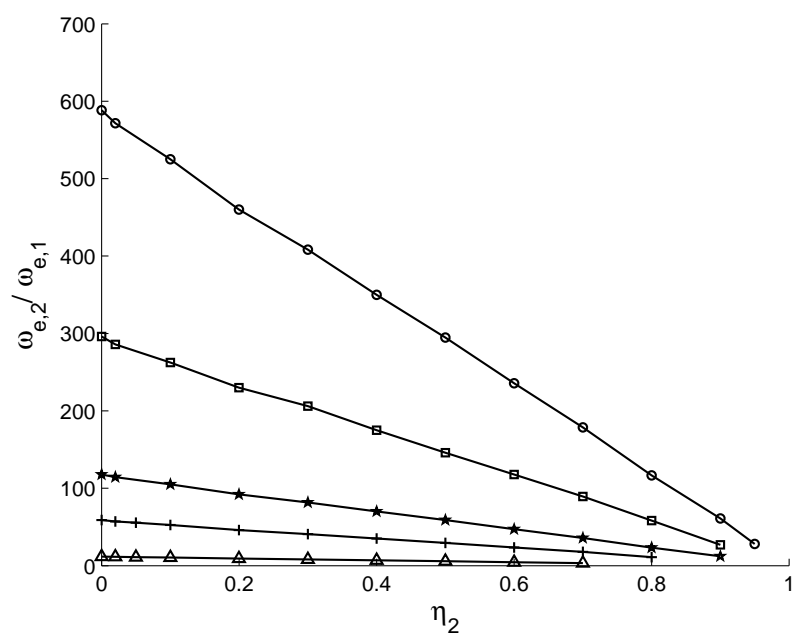

Figure 6.6: $A D U$ design plot. Minimum required frequency ratios for an increasing $\xi_{e, 2}$, and $\xi_{e, 1}^{\max }=0.3$. The values of $\xi_{e, 2}: \circ: 0.001, \square: 0.002, \star: 0.005$, $+: 0.01, \triangle: 0.05$.

\subsubsection{Tuning active damping controller}

Confronted with the undesired high order dynamics of the active damping mechanism, it is crucial to know what the maximum feasible active damping for the target mode is. In other words, how much active damping can be obtained without destabilizing the first noncollocated mode in the loop.

Figure 6.7 shows the feasible $\varrho$ (which is described by (6.16)) for various $\omega_{e, 2} / \omega_{e, 1}$. This plot is made for $\xi_{e, 2}=0.001$, which means that the noncollocated mode is very lightly damped (the worst case in Figure 6.6 ). $\varrho=1$, indicated by the dotted horizontal line, corresponds to applying maximum active damping. The most important conclusion that can be drawn from Figure 6.7 is that despite a low relative damping and modest frequency of the first noncollocated mode, there is room for applying considerable active damping. Also, the beneficial effect of an antiresonance close to the resonance for the noncollocated mode is visible again.

This figure can also be used to tune the active damping controller, by using only the frequency of the first noncollocated mode. For instance, if the first noncollocated mode appears at $100 \cdot \omega_{e, 1}$, it is safe to apply $\varrho=0.15$. 


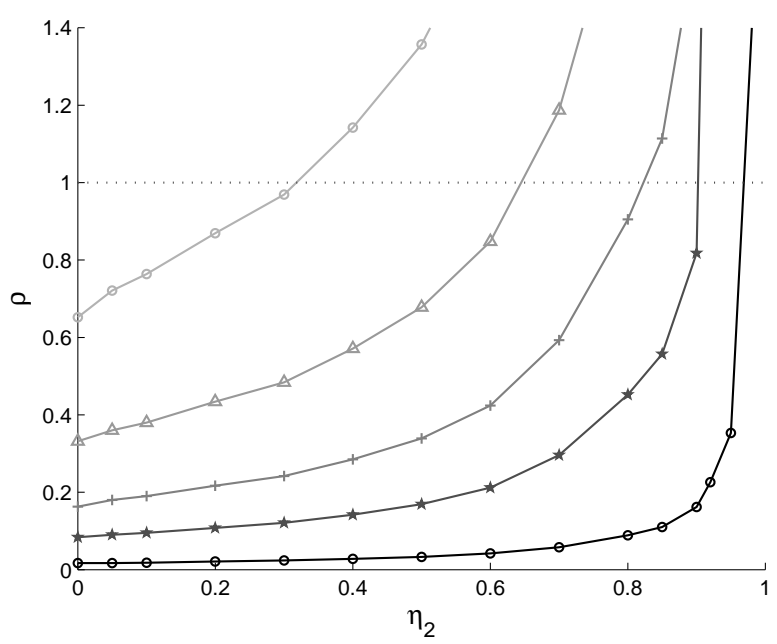

Figure 6.7: $A D U$ tuning plot. Maximum realizable $\varrho$, as a function of $\eta_{2}$ for various $\omega_{e, 2} / \omega_{e, 1}$. $\xi_{e, 2}=0.001$. From black to light gray: $\omega_{e, 2} / \omega_{e, 1}=$ $10,50,100,200$ and 400.

\subsection{Conclusions}

The stability bounds that apply for achievable active damping resulting from the first noncollocated mode of the active damping loop were presented in this chapter (see Fig 6.6 and Fig 6.7). These stability bounds are plotted as a function of $\xi_{e}$ (relative damping) and $\eta$ (relating resonance, $\omega_{e}$, to antiresonance, $\omega_{a}$ ) of the first noncollocated mode, in combination with its ratio to the target mode of the active damper.

Fig 6.6 provides guidelines and rules of thumb when designing an active damper unit. Fig 6.7 can be used for tuning the active damping controller for stability, knowing as little as only the frequency of the first noncollocated mode.

The danger of instability due to the noncollocated mode in the active damping loop is decreased considerably by adding just enough active damping to the target mode to meet the set requirements, instead of applying the maximum achievable active damping. 


\subsection{Discussion}

The value of $K$ corresponding to any point $s$ on a root locus can be calculated by:

$$
K_{s}=\frac{\prod_{i=1}^{m}\left(s-p_{i}\right)}{\prod_{i=1}^{n}\left(s-z_{i}\right)}
$$

So for the point $s_{o}$, being the point on the border of instability as shown in Figure 6.8, the following applies:

$$
\begin{aligned}
K_{s_{o}} & =\frac{\prod_{i=1}^{m}\left(s_{o}-p_{i}\right)}{\prod_{i=1}^{n}\left(s_{o}-z_{i}\right)} \\
& =K_{s_{o}, 1} \cdot \frac{\prod_{i=3}^{m}\left(s_{o}-p_{i}\right)}{\prod_{i=3}^{n}\left(s_{o}-z_{i}\right)} \\
& =K_{s_{o}, 1} \cdot K_{s_{o}, 2}
\end{aligned}
$$

where $K_{s_{o}, 1}$ only includes the target mode, $i=1$, and the first noncollocated mode, $i=2$, and $K_{s_{o}, 2}$ incorporated the modes between the two. Since the modes between the target mode and the first noncollocated mode are of the AR type,

$$
\left(s_{o}-z_{i}\right)>\left(s_{o}-p_{i}\right), \quad \forall i>2
$$

Hence:

$$
\begin{aligned}
K_{s_{o}, 2} & <1 \\
K_{s_{o}} & <K_{s_{o}, 1}
\end{aligned}
$$

which in general is true, since commonly multiple collocated modes exist in between the two considered modes. This means that the design guidelines derived in this chapter are rather optimistic.

The contribution of the modes beyond $\omega_{e, 2}$ to $K_{s_{o}}$ could be smaller, equal or greater than 1, depending on the mode types (see Table 6.T). So their effect can not be incorporated in the design guidelines without any knowledge about the situation at hand. 


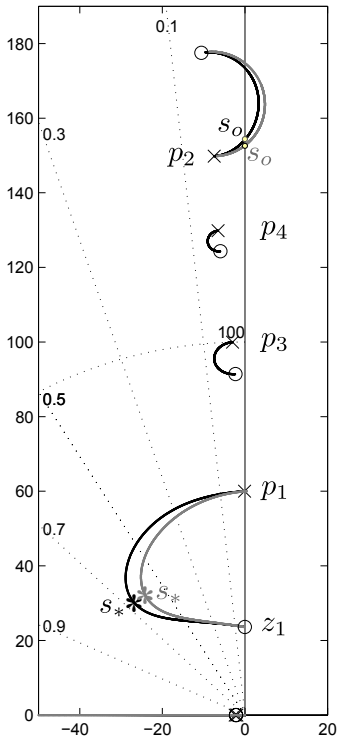

(a)

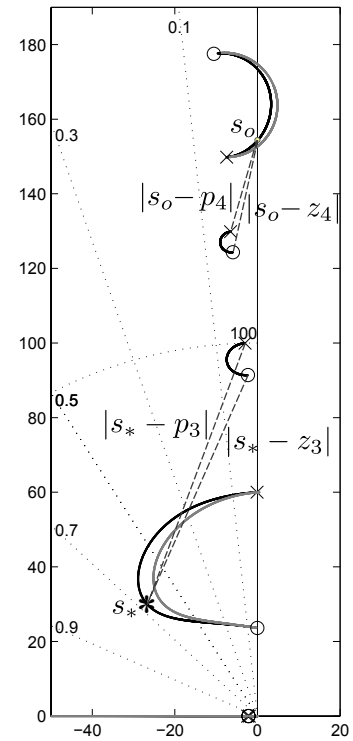

(b)

Figure 6.8: The effect of multiple collocated modes between the target mode and the first noncollocated mode on the AVC-loop root-locus (black linee), compared to the analyzed situation incorporating only the target mode and the first noncollocated mode (gray lines). 


\section{Chapter 7}

\section{Conclusions and Recommendations}

In light of the goals set for this project, as described in Section $\mathbb{1 . 1}$, this chapter reflects on the results achieved in Section $\square]$, and on the work that still needs to be done in Section 7.2 .

\subsection{Conclusions}

This section summarizes the main conclusions of the previous chapters.

\section{Design guidelines for a stable control loop}

The characteristics of an arbitrary eigenmode that have a significant influence on the closed-loop stability are identified to be the frequency of that mode, its damping ratio, and its controllability. The controllability of a vibration mode refers to the distance between the corresponding pole and zero; The greater the distance between the two, the higher the controllability of that mode. These parameters constitute the type of a certain mode. For several controller configurations, stability bounds have been determined for various types of highorder modes.

Choosing the low authority/high authority control strategy results in two independent control loops that operate in parallel. Their stability can hence be analyzed separately. The so-called stability regions have been computed for the two types of controllers implemented in the motion-control loop and the activedamping loop, in this thesis. The results are depicted in various plots that can be found in Chapter $\square$ (Figures $\sqrt{2.4}$ and $[2.6$ ) and Chapter $\mathbf{6}$ (Figures 6.5 and 5.6 ). These plots show the stability robustness that can be gained by damping in motion systems, and give a set of guidelines that can be used for the mechatronic design of a control loop. The term "design", can relate to the choice of the 
controller bandwidth, tuning the controller parameters, the choice of the actuator and sensor on the basis of their dynamics, and the specifications belonging to any mechanism embedding the actuator and sensor.

\section{Collocated and noncollocated active damping in motion systems}

The presumption that forms the basis of this research is that the performance of the closed-loop motion system can be improved by adding damping to the resonance modes of the plant. Chapter $\mathbf{B}$ showed that this hypothesis does not simply apply for at least one class of noncollocated active damping.

When the same actuator is used in both the motion-control loop and activedamping loop, no damping is added to the zeros of the motion-control loop. This can readily be seen from the transfer function of the plant in the motioncontrol loop, when the active-damping loop is closed. Since the poles move toward the same poorly damped zeros, as in the case of no active damping, for an increasing motion-control bandwidth, the resonance mode starts to exhibit oscillatory behavior, which might worsen the performance of the closed-loop system.

Collocated active damping increases the damping of both the poles and the zeros of the motion-control loop, keeping the poles of the motion-control loop well damped for an increasing bandwidth. This makes the performance improvement by increasing the motion-control bandwidth possible, which was aimed for by active damping.

The increase of damping of the zeros belonging to the motion-control loop is an important advantage of collocated active vibration control when it comes to performance improvement of motion systems.

\section{One-dimensional active damping}

The measurements presented is this thesis were performed using the Rocking Mode Demonstrator (RMD). The RMD is a test setup, demonstrating the effect of the rocking mode on the dynamics of the end effector. To add damping to the rotational vibration mode of this setup, an Active Damping Unit (ADU) has been realized. First a set of requirements for the ADU with reference to the rotational vibration mode to be damped, were determined. These requirements are translated into a mechanical design. However, the dynamics of the realized ADU differ from the prediction, resulting in low controllability of the vibration mode.

The low controllability of the target vibration mode has been improved by implementing the so-called static response compensation. This compensation can result in non-minimum phase zeros in the active-damping loop, making this control loop less robust against parameter variations. 
The measurements clearly show the beneficial effect of the added damping in the end-effector behavior. The increase of the motion-control bandwidth could however not be demonstrated. The first resonance mode, simulating the rocking mode, does not destabilize the motion-control loop because of its relatively high damping. In fact, it is the second vibration mode in the plant transfer of the RMD that limits the achievable motion-control bandwidth. However, the low-pass dynamics of the actuator amplifier in the active-damping loop prevents addition of damping to this vibration mode of the setup.

\section{The three-dimensional effect of modal damping on the end effector}

This thesis presented a competent model of the effect of modal active vibration control on the three-dimensional end-effector position, in terms of Euclidean coordinates. This general model is derived from the geometry of the plant, using the screw theory, and can be applied for other cases of active vibration control in motion systems.

Using this model, it was shown that a small deviation of the active vibration control axis and the actual vibration axis of the end effector has a rather small influence on the achievable damping. Hence, it can be disregarded in that sense. However, this deviation does introduce dynamics in other degrees of freedom of the end effector. So these newly introduced dynamics might worsen the performance.

\section{Overall conclusions}

To recap, the goal of this research was formulated as investigating the addition of damping to the rotational vibration mode of a linearly actuated motion system to

- achieve a shorter settling time in the transient response of the plant to a commanded motion

- increase the achievable closed-loop motion-control bandwidth to obtain an improved performance

This thesis started by showing the role of damping on the stability of motion systems, for P(I)D-type motion controllers. The root-locus analysis validates the increase of the bandwidth resulting from the increase in modal damping to various extents for different types of high-order modes.

The practical implementation of active damping in a setup designed with a rotational vibration mode showed the performance improvement by damping in the transient behavior of the plant.

The increase of the bandwidth as a result of active damping could not be demonstrated using the current test setup, because of the relatively high damping already present in the setup. 


\subsection{Recommendations}

A typical challenge when implementing active damping in motion systems is that the resonance frequency of the end effector can change by changes in the geometry. For instance, the extension of the arm of a pick-and-place machine changes the inertia of the end effector, leading to variation of its resonance frequency during operation. So the effect of this resonance shift should be accounted for when tuning the AVC. In view of this parameter variation, the AVC should be tuned such that sufficient damping is added to the resonance mode over the entire frequency range it can occur in.

The maximum achievable damping depends on the ratio between the resonance and antiresonance frequencies of that mode. By placing the collocated active damping unit as close as possible to the guideway, the variation in the antiresonance frequency of the active-damping loop will follow the same trend as the resonance frequency variation. After all, the antiresonance frequency is the resonance frequency of the subsystem constrained by the actuator and sensor of the active-damping loop. By making this subsystem resemble the resonating load as much as possible, the changes in the parameters affecting the resonance frequency will affect the antiresonance frequency at the same time. Consequently, the root-locus of the target mode will roughly preserve its shape, leaving the maximum achievable damping almost unaffected (depending on the extent of the dynamics captured by the antiresonance, as already mentioned). The gain for which the maximum damping can be achieved, however, depends on both the ratio between the resonance and antiresonance frequency, as well as the resonance frequency. Since the aforementioned ratio remains quite constant, by only taking the variation of the resonance frequency into account, the active vibration controller can be tuned for acceptable damping over the entire vibration-frequency range. This could for instance be done by appropriate gain scheduling or using an optimal gain resulting from an appropriate cost function. It is hence interesting and relevant to investigate this and to come up with an appropriate tuning procedure.

Although the chosen control structure and algorithm of the active damping are proven to be effective, the design of the active damping unit remains a challenge. One aspect of the active damping mechanism that should be investigated further is its stiffness in other directions than its degree of freedom. The analysis in Chapter $\square$ was performed under the assumption that the active damping unit is infinitely stiff in all directions, but in its own degree of freedom This is in general not the case. The addition of the ADU can in fact decrease the stiffness in the other directions, which can have a negative effect on the performance of the end effector. 
Another challenge when realizing an active damping unit is the resulting controllability of the target mode, which basically refers to the ratio between the resonance and antiresonance frequencies of that mode. The current realization of the ADU results in a low controllability of the rotational vibration mode of the RMD. This is inconsistent with the predictions from the models of the ADU. The model of the mechanical concepts implemented in the ADU should be validated to find out the cause of difference between the expectations and the actual properties of the ADU. Specially the model of the stroke amplification mechanism should be validated. An adequate model of the ADU is not only useful for the current implementation of active damping, but also for utilizing any concept used in the current mechanism in another design. The ADU should be disassembled as well as possible and examined more closely to find out the reason for the difference between the expectations and the actual properties of the ADU. The piezo stack should be tested for electrical leakage (between the sensor and actuator) and mechanical coupling (deformation of the sensor resulting from the actuation of the actuator). Also both the stroke of the stroke amplifying mechanism and the forces it generates should be tested to validate the expected behavior and the parameters deduced from the FEM analysis. 


\section{Appendix A}

\section{Detailed mechanical design of the ADU}

This appendix is contributed by Dr. ir. D. M. Brouwer.

A flexure mechanism has been designed which converts the $20 \mu \mathrm{m}$ translational motion of the piezo's into a $0.70^{\circ}$ rotation. To make the mechanism repeatable (Eastman 1935) and deterministic (Hale 1999, Soemers 2010) the design is based on flexures to eliminate backlash and minimize hysteresis and friction. The mechanism consist of a range amplification mechanism and a translation to rotation converter.

Each piezo actuator has a range amplifier mechanism based on inclined beams, shown in Figure A.1. An integrated preload adjustment screw assures that the piezo is loaded compressively at all times. The stroke amplification and stiffness of the amplification mechanism are calculated in the next section. The amplification mechanism can be observed as four inclined beams, two in parallel and two in series. Therefore the stroke and stiffness are equal to the single inclined beam, shown in Figure A.2, with one end fixed, the other end fixed guided. The elongation $y^{\prime}$ as a function of displacements $x$ and $y$ of the beam is

$$
y^{\prime}=x \sin \left(v_{0}\right)+y \cos \left(v_{0}\right)
$$

The displacement in bending direction, $x^{\prime}$, as a function of displacements $x$ and $y$ of the beam is

$$
x^{\prime}=x \cos \left(v_{0}\right)-y \sin \left(v_{0}\right)
$$

Dual to the displacements the forces can be converted

$$
P=P^{\prime} \cos \left(v_{0}\right)-W^{\prime} \sin \left(v_{0}\right)
$$




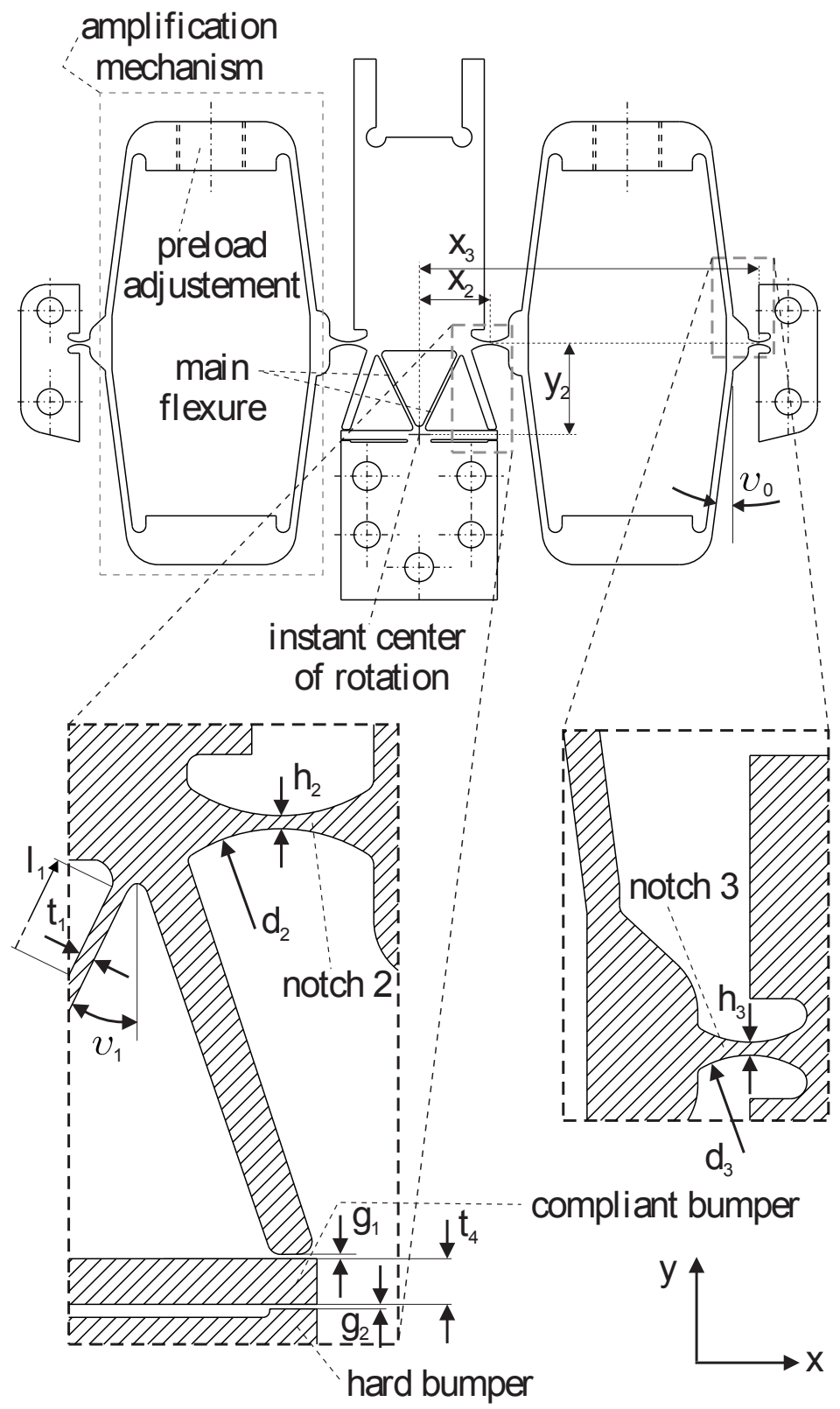

Figure A.1: Drawing view of the Active Damper Unit, top view. 
and

$$
W=-P^{\prime} \sin \left(v_{0}\right)+W^{\prime} \cos \left(v_{0}\right)
$$

The axial force in the beam becomes

$$
P^{\prime}=\frac{E A}{l_{0}} y^{\prime}
$$

with $E$ the Young's modulus, $A=w_{0} \cdot t_{0}$ the cross-sectional area of the beam, $w_{0}$ is the width of the beam and the thickness of the plate in which the mechanism is fabricated, and $t_{0}$ is the thickness of the beam. The bending force in the beam becomes

$$
W^{\prime}=\frac{12 E I}{l_{0}^{3}} x^{\prime}
$$

with $I=w_{0} \cdot t_{0}^{3} / 12$ the second moment of area.

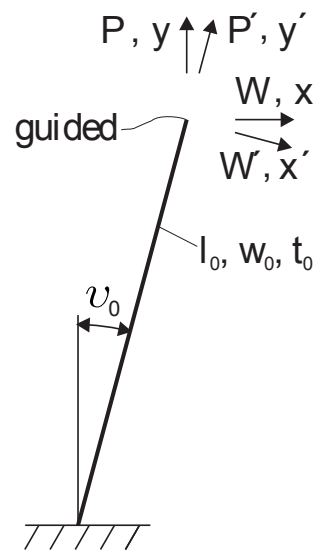

Figure A.2: Deflection and forces of an inclined beam with one end fixed, the other end guided.

The stiffness $k_{x}$ in $x$-direction with the piezo actuator blocked is

$$
k_{0}=\left.\frac{d W}{d x}\right|_{y=0}=\frac{E A}{l_{0}} \sin ^{2}\left(v_{0}\right)+\frac{12 E I}{l_{0}^{3}} \cos ^{2}\left(v_{0}\right)
$$

The stroke amplification becomes

$$
i=\left.\frac{d x}{d y}\right|_{W=0}=\left|\frac{\frac{E A}{l_{0}} \cos \sin \left(v_{0}\right)+\frac{12 E I}{l_{0}^{3}} \cos \sin \left(v_{0}\right)}{\frac{E A}{l_{0}} \sin ^{2}\left(v_{0}\right)+\frac{12 E I}{l_{0}^{3}} \cos ^{2}\left(v_{0}\right)}\right|
$$


With the dimensions tabulated in Table A.1 the stiffness of the stroke amplification mechanism, $k_{0}=d W / d x$, becomes $1.6 \cdot 10^{6} \mathrm{~N} / \mathrm{m}$ and the stroke amplification, $d x / d y$, becomes 6.9. With the Piezo stiffness, $250 \cdot 10^{6} \mathrm{~N} / \mathrm{m}$, chipsensor stiffness, $1900 \cdot 10^{6} \mathrm{~N} / \mathrm{m}$, and the distance $y_{2}$ the stackstiffness of two stacks becomes $98.5 \mathrm{Nm} / \mathrm{rad}$. A FEM model however showed a stroke amplification factor of 6.3 and a stiffness of $82 \mathrm{Nm} / \mathrm{rad}$. The difference is caused by the parasitic stiffnesses in the FEM model which add extra compliance. The longitudinal stiffness of the notch hinges and the stiffness in $x$-direction of the main flexure are several orders higher than stiffness of the stack, and are therefore not taken into account in $k_{s}$.

Table A.1: dimensions of the ADU.

\begin{tabular}{|c|cc|}
\hline \hline Dimension & Value & Unit \\
\hline$v_{0}$ & 7.4 & $\circ$ \\
$v_{1}$ & 26 & $\circ$ \\
$a$ & 1.17 & - \\
\hline$d_{2}$ & 5.0 & $\mathrm{~mm}$ \\
$d_{3}$ & 3.0 & $\mathrm{~mm}$ \\
\hline$g_{1}$ & 0.07 & $\mathrm{~mm}$ \\
$g_{2}$ & 0.07 & $\mathrm{~mm}$ \\
\hline$h_{2}$ & 0.20 & $\mathrm{~mm}$ \\
$h_{3}$ & 0.20 & $\mathrm{~mm}$ \\
\hline$l_{0}$ & 12.5 & $\mathrm{~mm}$ \\
$l_{1}$ & 5.8 & $\mathrm{~mm}$ \\
$t_{0}$ & 0.55 & $\mathrm{~mm}$ \\
$t_{1}$ & 0.24 & $\mathrm{~mm}$ \\
$w_{0}$ & 10.0 & $\mathrm{~mm}$ \\
\hline$x_{2}$ & 3.1 & $\mathrm{~mm}$ \\
$x_{3}$ & 2.5 & $\mathrm{~mm}$ \\
$y_{2}$ & 6.5 & $\mathrm{~mm}$ \\
\hline \hline
\end{tabular}

The amplification mechanism loads the beam laterally. In addition, in the case of overload, a sideways collision will load the main flexure hinge laterally too. Therefore, the main flexure has to withstand the lateral loads of actuation and collision, and axial load of the mass of the attached beam. A main flexure design is proposed that allows for wire electro discharge machining in one monolithic part, has five stiffly supported degrees of freedom, and the rotation compliant. The main flexure hinge consists of two leaf springs, shown in Figure A.1] and Figure A.3. The intersection of the leaf springs creates an instant center of 


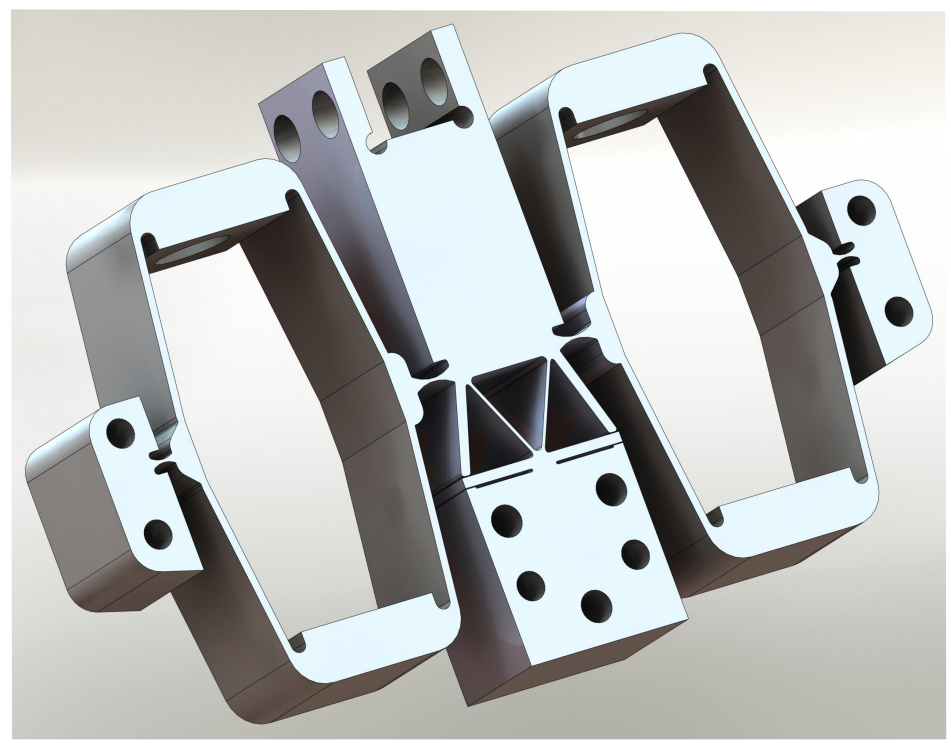

Figure A.3: 3-D view of the Active Damper Unit.

rotation. Stiffness wise these leaf springs act in parallel. Hinges 2 and 3 are mainly loaded axially, and therefore these can conveniently be designed as notch hinges.

The rotations of the main flexure and notch hinges are calculated.

$$
\tan \left(\Delta v_{3}\right)=\frac{y_{2}-l_{2} \cos \left(v_{02}+\Delta v_{1}\right)}{x_{2}-l_{2} \sin \left(v_{02}+\Delta v_{1}\right)}
$$

with

$$
\tan v_{02}=\frac{x_{2}}{y_{2}} ; \quad l_{2}=\sqrt{x_{2}^{2}+y_{2}^{2}}
$$

in which $x_{2}, x_{3}$ and $y_{2}$ are shown in Figure A.1, $\Delta v_{1}$ and $\Delta v_{3}$ are the rotational displacements of respectively the main flexure and notch hinge 3 . The input dimensions and results are tabulated in Table A.1 and A.2 respectively. The rotational displacement of the second flexure $\Delta v_{2}$ can then be calculated

$$
\Delta v_{2}=\Delta v_{1}+\Delta v_{3}
$$

The combined rotational stiffness of the flexure mechanism, $k_{p l}$ without piezo's and piezo amplification mechanism is a combination of the stiffness of the main flexure $k_{1}$, and notch hinges 2 and 3 , respectively $k_{2}$ and $k_{3}$

$$
k_{\mathrm{pl}}=k_{1}+2\left(\frac{\Delta v_{1}}{\Delta v_{2}}\right)^{2} k_{2}+2\left(\frac{\Delta v_{1}}{\Delta v_{3}}\right)^{2} k_{3}
$$


Table A.2: rotations, stiffnesses and stresses of the ADU.

\begin{tabular}{|c|cc|}
\hline \hline Parameter & Value & Unit \\
\hline$k_{s}(\mathrm{FEM})$ & $98.5(82)$ & $\mathrm{Nm} / \mathrm{rad}$ \\
$i$ (FEM) & $6.9(6.3)$ & - \\
\hline$\Delta v_{1}$ & 0.7 & $\circ$ \\
$\Delta v_{2}$ & 0.8 & $\circ$ \\
$\Delta v_{3}$ & 0.1 & $\circ$ \\
\hline$k_{1}$ & 5.2 & $\mathrm{Nm} / \mathrm{rad}$ \\
$k_{2}$ & 1.5 & $\mathrm{Nm} / \mathrm{rad}$ \\
$k_{3}$ & 1.9 & $\mathrm{Nm} / \mathrm{rad}$ \\
$k_{p l}$ & 9.2 & $\mathrm{Nm} / \mathrm{rad}$ \\
\hline \hline
\end{tabular}

The rotational stiffness of the main flexure, $k_{1}$, around its instant center of rotation can be approximated using the Euler-Bernoulli beam equations which leads to (van Eijk 1985)

$$
k_{1}=8\left\{\frac{E I}{l_{1}}\left(1-3 a+3 a^{2}\right)\right\}
$$

in which $l_{1}$ is the length of the leaf springs, and $a \cdot l_{1}$ is the distance from the instant center of rotation to the far end of the leaf spring. The notch hinges are preferably loaded in tensile direction. A notch hinge then combines a high axial stiffness, rotational compliance, low bending stress and loadability. The notch hinges can be dimensioned based on Koster (1996). The rotational stiffness of notch hinge $i$ with $i=2,3$ is approximated by

$$
k_{i}=0.093 \sqrt{\frac{h_{i}}{d_{i}}} E w_{0} h_{i}^{2}
$$

With the dimensions listed in Table A.1 $k_{p l}$ becomes $9.2 \mathrm{Nm} / \mathrm{rad}$.

The maximum bending stress of the main flexure is given by van Eijk (1985) and amounts to $257 \mathrm{~N} / \mathrm{mm}^{2}$. The bending stress in the notch hinges is given by Koster (1996) is $325 \mathrm{~N} / \mathrm{mm}^{2}$ and $57 \mathrm{~N} / \mathrm{mm}^{2}$ in hinges 2 and 3 respectively.

Because the main flexure is loaded by a distant mass resulting in a moment, elastic stability has to be checked. Preferably the critical moment is several times higher than the moment acting on the leaf springs. The main flexure is designed such that the critical moment is three times higher than the intended load on the flexure.

To protect the flexures from over loading by the actuators, a compliant bumper is introduced. When the rotation, $\Delta v_{1}$ exceeds the allowable value, gap $g_{1}$ is reduced to zero and the compliant bumper is collided with. The stiffness of this bumper is 10 times the rotational stiffness of the flexure. This way the collision 
forces are limited. A second bumper with large stiffness stops the rotational motion entirely, after running through gap $g_{2}$.

The ADU is made out of Stavax Supreme, a high yield mold steel, $E=200 \mathrm{GPa}$ and $G=76.9 \mathrm{GPa}$. The dimensions and results are tabulated in Table $\mathrm{A.1}$ and $\mathrm{A.2}$ respectively. 


\section{List of publications}

\section{Journal papers}

- Babakhani, B., de Vries, T. J. A. \& van Amerongen, J. (2012a), 'A comparison of the performance improvement by collocated and noncollocated active damping in motion systems', IEEE/ASME transactions on Mechatronics to be published.

- Babakhani, B., Brouwer, D. M., de Vries, T. J. A. \& van Amerongen, J. (2012), 'Experimental evaluation of active damping of a rotational vibration mode in motion systems', submitted to IEEE/ASME Transactions on Mechatronics.

- Babakhani, B., de Vries, T. J. A. \& van Amerongen, J. (2012), 'The Role of Damping on the Stability of P(I)D-controlled motion systems', submitted to Mechatronics.

\section{Conference papers}

- Babakhani, B., de Vries, T. J. A. \& van Amerongen, J. (2012c), Off-axis modal active vibration control of roational vibrations, in 'Proceedings of the IEEE/ASME International Conference on Advanced Intelligent Mechatronics (AIM)', pp. 326-331.

- Babakhani, B., de Vries, T. J. A. \& van Amerongen, J. (2012b) , Design guidelines concerning the destabilizing high-frequency mode of an integral force feedback-based active damping unit, in 'Proceedings of the IEEE/ASME International Conference on Advanced Intelligent Mechatronics (AIM)', pp. 520-525.

- Babakhani, B. \& de Vries, T. J. A. (2010b), On the stability properties of PIDcontrolled motion systems, in 'Proceedings of the 5th IFAC Symposium on Mechatronic Systems', pp. 473-477.

- Babakhani, B. \& de Vries, T. J. A. (2010), Active damping of the 1D rocking mode, in 'Proceedings of the IEEE International Conference on Mechatronics and Automation (ICMA)', pp. 1370-1375. 


\section{Abstracts}

- Babakhani, B. \& de Vries, T. J. A. (2010), Active damping in precision equipment using piezo, in '29th Benelux Meeting on Systems and Control', pp. 74.

- Holterman, J., de Vries, T. J. A., Babakhani, B. \& Brouwer, D. M. (2012), 'Design of a piezoelectric rotation actuator', in 'Electroceramics XIII', pp. 92. 


\section{Bibliography}

Altintas, Y. \& Okwudire, C. E. (2008), 'Dynamic stiffness enhancement of directdriven machine tools using sliding mode control with disturbance recovery', CIRP Annals - Manufacturing Technology 58(1), 335-338.

Anderson, E. H. \& Hagood, N. W. (1994), 'Simultaneous piezoelectric sensing/actuation: analysis and application to controlled structures', Journal of Sound and Vibration 174(5), 617-639.

Aphale, S. S., Fleming, A. J. \& Moheimani, S. O. R. (2007), 'Integral resonant control of collocated smart structures', Smart Materials and Structures 16, 439446.

Åström, K. J. (2008), Feedback Systems, Princeton University Press, New Jersey.

Åström, K. J. \& Murray, R. M. (2001), 'The future of PID control', Control Engineering Practice 9, 1163-1175.

Babakhani, B., Brouwer, D. M., de Vries, T. J. A. \& van Amerongen, J. (2012), 'Experimental evaluation of active damping of a rotational vibration mode in motion systems', submitted to IEEE/ASME Transactions on Mechatronics .

Babakhani, B. \& de Vries, T. J. A. (2010a), Active damping of the 1d rocking mode, in 'Proceedings of the IEEE International Conference on Mechatronics and Automation (ICMA)', pp. 1370-1375.

Babakhani, B. \& de Vries, T. J. A. (2010b), On the stability properties of p(i)dcontrolled motion systems, in 'Proceedings of the 5th IFAC Symposium on Mechatronic Systems', pp. 473-477.

Babakhani, B., de Vries, T. J. A. \& van Amerongen, J. (2012a), 'A comparison of the performance improvement by collocated and noncollocated active damping in motion systems', IEEE/ASME transactions on Mechatronics to be published.

Babakhani, B., de Vries, T. J. A. \& van Amerongen, J. (2012b), Design guidelines concerning the destabilizing high-frequency mode of an integral 
force feedback-based active damping unit, in 'Proceedings of the IEEE/ASME International Conference on Advanced Intelligent Mechatronics (AIM)', pp. 520-525.

Babakhani, B., de Vries, T. J. A. \& van Amerongen, J. (2012c), Off-axis modal active vibration control of roational vibrations, in 'Proceedings of the IEEE/ASME International Conference on Advanced Intelligent Mechatronics (AIM)', pp. 326-331.

Balas, M. J. (1979), 'Direct velocity feedback control of large space structures', Journal of Guidance, Control, and Dynamics 2(3), 252-253.

Ball, R. S. (1900), A Treatise in the Theory of Screws, Cambridge University Press.

Baz, A. \& Poh, S. (1996), 'Optimal vibration control with modal positive position feedbac', Optimal Control Applications \& Methods 17(2), 141-150.

Berkhoff, A. P. \& Wesselink, J. M. (2011), 'Combined mimo adaptive and decentralized controllers for broadband active noise and vibration control', Mechanical Systems and Signal Processing 25(5), 1702-1714.

Borger, D. (2010), On the stability properties of motion controllers, Master's thesis, University of Twente, Enschede, The Netherlands.

Borger, D. \& Holterman, J. (2010), Rocking mode demonstrator, Technical report, Imotec b.v.

Bos, A. M. (1986), Modeling Multibody Systems in terms of Multibond Graphs, PhD thesis, University of Twente, Enschede, The Netherlands.

Breedveld, P. C. (1985), 'Multibond graph elements in physical systems theory', Journal of the Franklin Institute 319(1/2), 1-36.

Chen, Y. \& Tlusty, J. (1995), 'Effect of low-friction guideways and lead-screw flexibility on dynamics of high-speed machines', CIRP Annals - Manufacturing Technology 44(1), 353-356.

Choi, S. B., Han, S. S., Kim, H. K. \& Cheong, C. C. (1999), ' $\mathrm{H}_{\infty}$ control of a flexible gantry robot arm using smart actuators', Mechatronics 9, 271-286.

Clark, W. W. \& Robertshaw, H. H. (1997), 'Force feedback in adaptive trusses for vibration isolation in flexible structures', Journal of Dynamic Systems, Measurement, and Control 119, 365-371.

Coelingh, H. J. (2000), Design Support for Motion Control Systems, PhD thesis, University of Twente, Enschede, The Netherlands. 
Coelingh, H. J., de Vries, T. J. A. \& Koster, R. (2002), 'Assessment of mechatronic system performance at an early design stage', IEEE/ASME Transactions on Mechatronics 7(3), $269-279$.

de Roover, D. (1997), Motion control of a wafer stage, PhD thesis, Delft University of Technology, Delft, The Netherlands.

de Silva, C. W. (2000), Vibration Fundamentals and Practice, CLC Press, USA.

Denoyer, K. K. \& Kwak, M. K. (1996), ‘Dynamic modelling and vibration suppression of a slewing structure utilizing piezoelectric sensors and actuators', Journal of Sound and Vibration 189(1), 13-31.

Duindam, V., Macchelli, A., Stramigioli, S. \& Bruyninckx, H. (2009), Modeling and Control of Complex Physical Systems, The Port-Hamiltonian Approach, Springer, Heidelberg, Germany.

Eastman, F. S. (1935), Flexure pivots to replace knife edges and ball bearings, Technical Report Engineering Experiment Station Bulletin 86, University of Washington.

Ehmann, C. (2002), Robust controller synthesis vs. integral force feedback with collocation for active damping of flexible structures, in 'Proceedings ISMA; International Conference on Noise and Vibration Engineering'.

Fleming, A. J. (2010), 'Nanopositioning system with force feedback for highperformance tracking and vibration control', IEEE/ASME Transactions on Mechatronics 15(3), 433-447.

Flow International Corporation (2012), 'Flow WMC2 - waterjet machining center'. www.flowcorp.com.

Franklin, G. F., Powell, J. D. \& Emani-Naeini, A. (2006), Feedback Control of Dynamic Systems, Pearson Prentice Hall, New Jersey.

Fritsch GmbH (2012), 'Semi-automatic manipulator SM 902'. http://www.fritsch-smt.de.

Fuller, C. R. (1997), Active Control of Vibration, Academic press limited, London.

Gawronski, W. K. (2004), Advanced Structural Dynamics and Active Control of Structures, Springer-Verlag, New York.

Geng, Z. J. \& Haynes, L. S. (1994), 'Six degree-of-freedom active vibration control using the stewart platforms', IEEE Transactions on Control Systems Technology 2(1), 45-53. 
Goh, C. J. \& Caughey, T. K. (1985), 'On the stability problem caused by finite actuator dynamics in the collocated control of large space structures', International Journal of Control 41(3), 787-802.

Hagood, N. W. \& von Flotow, A. (1991), 'Damping of structural vibrations with piezoelectric materials and passive electrical networks', Journal of Sound and Vibration 146(2), 243-268.

Hahn, H. (1981), Higher Order Root-locus Technique with Applications in Control System Design, Freidr. Vieweg \& Sohn Verlagsgesellschaft mbH, Germany.

Hale, L. C. (1999), Principles and Techniques for Designing Precision Machines, $\mathrm{PhD}$ thesis, University of California, Livermore, CA.

Harris, C. M. (1988), Shock and Vibration Handbook, McGraw-Hill, New York, USA.

Hegewald, T. \& Inman, D. J. (2001), 'Vibration suppression via smart structures across a temperature range', Journal of Intelligent Material Systems and Structures 12, 191-203.

Holterman, J. (2002), Vibration Control of High-Precision Machines with Active Structural Elements, PhD thesis, University of Twente, Enschede, The Netherlands.

Holterman, J. (2010), Active damping unit for the rocking mode demonstrator, Technical Report 2010.1505.V, Imotec b.v.

Holterman, J. \& de Vries, T. J. A. (2004), 'Active damping within an advanced microlithography system using piezoelectric smart discs', Mechatronics 14(1), 1534.

Holterman, J. \& de Vries, T. J. A. (2005), 'Active damping based on decoupled collocated control', IEEE/ASME Transactions on Mechatronics 10(2), 135-145.

Holterman, J. \& Groen, P. (2012), An Introduction to Piezoelectric Materials and Components, Stichting Applied Piezo, The Netherlands.

Inman, D. J. (2001), 'Active modal control for smart structures', Philosophical Transactions: Mathematical, Physical and Engineering Sciences 359(1778), 205-219.

Jiang, L. \& Miles, R. N. (1999), 'A passive damper for the vibration modes of the head actuator in hard disk drives', Journal of Sound and Vibration 220(4), 683694.

Kalman, R. E. (1960), On the general theory of control systems, in 'Proceedings of the 1st World Congress of the International Federation of Automatic Control', pp. 481-492. 
Karnopp, D. C., Margolis, D. L. \& Rosenberg, R. C. (2006), System Dynamics; Modeling and Simulation of Mechatronic Systems, John Wiley \& Sons, Inc., New Jersey.

Keane, A. J. \& Bright, A. P. (1996), 'Passive vibration control via unusual geometries: Experiments on model aerospace structures', Journal of Sound and Vibration 190(4), 713-719.

Koster, M. P. (1996), Constructieprincipes voor het Nauwkeurig Bewegen en Positioneren, Uitgeverij Universiteit Twente, Enschede, the Netherlands.

MacMartin, D. G. (1995), Collocated structural control: motivation and methodology, in 'Proceedings on the 4th IEEE Conference on Control Applications', pp. 1092-1097.

Mahmoodi, S. N., Ahmadian, M. \& Inman, D. J. (2010), 'Adaptive modified positive position feedback for active vibration control of structures', Journal of Intelligent Material Systems and Structures 21(6), 571-580.

Manning, W. J., Plummer, A. R. \& Levesley, M. C. (2000), 'Vibration control of a flexible beam with integrated actuators and sensors', Smart Materials and Structures 9(6), 932-939.

Mead, D. J. (1998), Passive Vibration Control, John Wiley \& Sons, Chichester, UK.

Mei, C., Mace, B. R. \& Jones, R. W. (2001), ‘Hybrid wave/mode active vibration control', Journal of Sound and Vibration 247(5), 765-784.

Meirovitch, L. (1986), Elements of Vibration Analysis, McGraw-Hill, Singapore.

Meirovitch, L. (1997), Principles and Techniques of Vibration, Prentice-Hall, Inc., New Jersey, United States of America.

Miu, D. K. (1992), Mechatronics, Electromechanics and Contromechanics, an Introduction, Springer-Verlag, Berlin.

Miu, D. K. (1994), 'On transfer function zeros of general colocated control systems with mechanical flexibilities', Journal of Dynamic Systems, Measurement, and Control 116, 151-154.

Mohamed, Z. \& Tokhi, M. O. (2004), 'Command shaping techniques for vibration control of a flexible robot manipulator', Mechatronics 14(1), 69-90.

Murphy, B. R. \& Watanabe, I. (1992), 'Digital shaping filters for reducing machine vibration', IEEE Transactions on Robotics and Automation 8(2), 285-289.

Omxie/SMTmax Corp. (2012), 'QM2100 automatic pick and place machine'. http://www.smtmax.com. 
Pereira, E., Aphale, S. S., Feliu, V. \& Moheimani, S. O. R. (2011), 'Integral resonant control for vibration damping and precise tip-positioning of a singlelink flexible manipulator', IEEE/ASME Transactions on Mechatronics 16(2), 232240.

Preumont, A. (1997), Vibration Control of Active Structures, Kluwer academic publishers, Dordrecht.

Preumont, A. (2006), Mechatronics, Dynamics of Electromechanical and Piezoelectric Systems, Springer, Dordrecht.

Preumont, A. \& Achkire, Y. (1997), 'Active damping of structures with guy cables', Journal of Guidance, Control and Dynamics 20(2), 320-326.

Preumont, A. \& Loix, N. (2000), 'Some perspectives of applications of active control to large space structures', 3667, 13-22.

Qiu, Z., Han, J., Zhang, X., Wang, Y. \& Wu, Z. (2009), 'Active vibration control of a flexible beam using a non-collocated acceleration sensor and piezoelectric patch actuator', Journal of Sound and Vibration 326(3-5), 438-455.

Rankers, A. M. (1997), Machine Dynamics in Mechatronic Systems, an Engineering Approach, PhD thesis, University of Twente, Enschede, The Netherlands.

Resato International b.v. (2012), 'ACM - resato waterjet technology'. http://www.resato.com/waterjet.

Rew, K., Han, J. \& Lee, I. (2002), 'Multi-modal vibration control using adaptive positive position feedback', Journal of Intelligent Material Systems and Structures 13, 13-22.

Rivin, E. I. (1999), Stiffness and Damping in Mechanical Design, Marcel Dekker, Inc., New York, Basel, USA.

Seltzer, S. M., Irwin, D., Tollison, D. \& Waites, H. B. (1988), An application of "high authority/low authority control" \& "positivity", Technical Report repost no. NASA TM-100338, NASA.

Singer, N. C. \& Seering, W. P. (1989), Design and comparison of command shaping methods for controlling residual vibration, in 'Proceedings of the IEEE International Conference on Robotics and Automation', Vol. 2, pp. 888-893.

Skogestad, S. (2004), 'Simple analytic rules for model reduction and PID controller tuning', Modeling, Identification and Control 25(2), 85-120.

Soemers, H. J. M. R. (2010), Design Principles for Precision Mechanisms, TPointprint, Enschede, the Netherlands. 
Soemers, H. M. J. R. \& de Vries, T. J. A. (2008), Lecture notes: Mechatronic Design of Motion Systems, University of Twente, Enschede, The Netherlands.

Stramigioli, S. (2001), Modeling and IPC Control of Interactive Mechanical Systems, A Coordinate-Free Approach, first edition edn, Springer-Verlag, Berlin.

Stramigioli, S. \& Bruyninckx, H. (2001), Geometry of dynamic and higher-order kinematic screw, in 'Proceedings of the IEEE International Conference on Robotics and Automation'.

Symens, W. (2004), Motion and Vibration Control of Mechatronic Systems with Variable Configuration and Local Non-linear Friction, PhD thesis, Katholieke Universiteit Leuven, Leuven, Belgium.

Symens, W., van Brussel, H. \& Swevers, J. (2003), Pole-placement vs. loopshaping design for gain-scheduling control of machine tools with position dependent dynamics, in 'Proceedings of European Control Conference', pp. 933-938.

Tjepkema, D., van Dijk, J. \& Soemers, H. M. J. R. (2012), 'Sensor fusion for active vibration isolation in precision equipment', Journal of Sound and Vibration 331(4), 735-749.

van der Poel, G. W. (2010), An Exploration of Active Hard Mount Vibration Isolation for Precision Equipment, PhD thesis, University of Twente, Enschede, The Netherlands.

van Dijk, J. \& Aarts, R. G. K. M. (2012), Analytical one parameter method for PID motion controller settings, in 'IFAC Conference on Advances in PID Control'.

van Eijk, J. (1985), On the Design of Plate Spring Mechanism, PhD thesis, Delft University of Technology, Delft, The Netherlands.

van Schothorst, G. (1999), Active vibration control using piezoelectric smart disks, in 'Proceedings of the SPIE Conference on Mathematics and Control in Smart Structures', Vol. 3667, pp. 637-648.

Verscheure, D., Paijmans, B., van Brussel, H. \& Swevers, J. (2006), Vibration and motion control design and trade-off for high-performance mechatronic systems, in 'Proceedings of the IEEE International Conference on Control Applications'.

Vervoordeldonk, M. J., van Eijk, J. \& Roozen, N. B. (2006), Active structural damping in high-precision equipment, in 'Proceedings of the International Conference on Noise and Vibration Engineering ISMA', pp. 573-581. 
Visser, L. C., Carloni, R. \& Stramigioli, S. (2009), Vision based motion control for a humanoid head, in 'Proceedings of the RSJ/IEEE International Conference on Intelligent Robots and Systems'.

Yang, B. (1994), 'Vibration control of gyroscopic systems via direct velocity feedback', Journal of Sound and Vibration 175(4), 525-534.

Zhou, L. \& Misawa, E. A. (2005), Low frequency vibration suppression shape filter and high frequency vibration suppression shape filter, in 'Proceedings of the American Control Conference', Vol. 7, pp. 4742-4747.

Zhou, Y., Peng, F. \& Li, B. (2008), 'Adaptive notch filter control for the torsion vibration in lead-screw feed drive system based on neural network', Lecture notes in Computer Science 5315(10), 803-812.

Ziegler, J. G. \& Nichols, N. B. (1942), 'Optimum settings for automatic controllers', Transactions of the ASME pp. pp. 759-768.

Zou, L. \& Slotine, J. E. (2005), 'Robust vibration isolation via frequency-shaped sliding control and modal decomposition', Journal of Sound and Vibration 285, 1123-1149. 


\section{Acknowledgment}

It has been a long time since I saw my aunt Golpar, whom I copy a lot, etch a circuit in the kitchen. This fascinating introduction to electrical engineering led to the choice of pursuing Electronic sciences. This thesis marks an important milestone in an intriguing journey yet to end. I am grateful for the help, support and enthusiasm of a lot of people around me. Here, I would like to thank a small group in particular.

First of all, I would like to thank my promotor prof. Job van Amerongen and my assistant promotor dr. Theo J. A. de Vries for giving me this opportunity, their guidance throughout the research, useful discussions, and of course, the concluding trajectory of writing this thesis during which they flexibly adapted to my tight schedule. Job, thank you for the motivating talks, critical questions and being my promotor, even after your retirement. Theo, your true involvement with my work has been a major help throughout the project. Your willingness to help even with the smallest technical details, your subtle constructive criticism and your compliments helped and motivated me to go on whenever I got stuck.

I am thankful to everybody who helped realizing the RMD setup, especially Dennis Borger, the only M.Sc. student I had the privilege to supervise, for his significant contribution to make the RMD operational, John Wesseler a.o. for the countless times he put the $\mathrm{ADU}$ in the RMD, just to remove it again on my request and Jan Holterman, who in addition to his help with the RMD setup, helped me throughout the project and reviewed a lot of my papers.

Johannes van Dijk and Dirk Tjepkema, I am grateful for our useful discussions during our meetings. I am specially indebted to you, Dirk, for your great help with investigating the unexpected behavior of the ADU and the useful SPACAR model you made.

I would like to thank all my colleagues for the pleasant working environment at the Robotics and Mechatronics group. Our coffee and lunch breaks, group events and parties were always very 'gezellig'. Though I am very glad to have finished my Ph.D., I am very sad to leave you! 
Thank you Carla, Jolanda, Alfred, Marcel and Gerben for all the administrative and technical support in the past years.

In particular, I would like to thank my roommates Rob, Ludo, Bart, Yury, Marcel, Douwe, Yunyun, Xiaochen, Abeje and Oğuzcan for all the fun and your involvement in the past years. Besides chitchats about everything and nothing, you guys helped a lot, every time I asked you. The most recent example is the cover design of this thesis, for which everybody, and in particular Yury, gave useful tips and comments. I have enjoyed our small talk, serious discussions, teasing one another and the board-game nights immensely :-)

Ludo and Abeje, special thanks to you, beside everything else, for your extensive technical support. I have bothered you quite a lot of times and you were always willing to help me with a friendly smile.

A big thank you to all the friends and family who have been a tremendous emotional support. Though you are not mentioned by name, I am truly thankful.

Entirely according to my habit, I have kept the best for last. Mom, Roonak and Bert, you are the ones I am the most indebted to. You have helped me in every way possible.

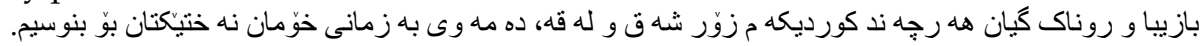

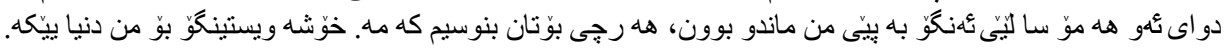

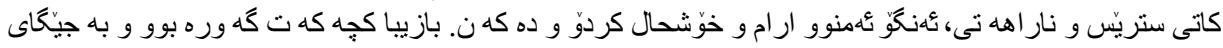

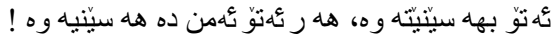

Lieve Bert, ik heb de laatste tijd van jou het meeste van allemaal gevraagd. Jij hebt mij met alle belangrijke beslissingen geholpen, stond zoals altijd met liefde voor mij klaar en hebt in drukke tijden zoveel mogelijk mijn lasten verlicht. Thank-you seems too simple a word to express my gratefulness, but it is all that I can say. Thank you, most of all, for making my home! 


\section{About the author}

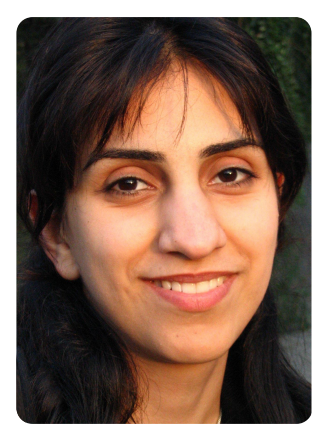

Bayan Babakhani was born in Teheran (Iran) on December 26th, 1982. After completing her high school at Scholengemeenschap Winkler Prins in 2001, she started her study in electrical engineering at the University of Twente, Enschede, The Netherlands.

Among various extracurricular activities during her study, she was the treasurer of the study tour project Shouraizou from 2003 to 2005. This project on technological innovations in Japan, concluded with a study tour to Japan visiting 20 different companies, universities and institutes.

After an internship at Philips TASS (Eindhoven) in 2006 on implementing an Electronic Stability Program (ESP, for the automotive industry) in an FPGA, she wrote her M.Sc. thesis on haptic teleoprators, under the supervision of prof. S. Stramigioli.

In 2008, after obtaining her M.Sc. degree, she started her Ph.D. at the Robotics and Mechatronics group of the University of Twente, under the supervision of prof. J. van Amerongen and dr. T. J. A. de Vries. Her Ph.D. research, within the framework of SmartPie research program, was on active damping of vibrations in high-precision motion systems. During her Ph.D., she successfully completed the educational program of the DISC Graduate School on Systems and Control. 


\section{Invitation}

to the public defense of my Ph.D. dissertation

Active Damping of Vibrations in High-Precision Motion Systems

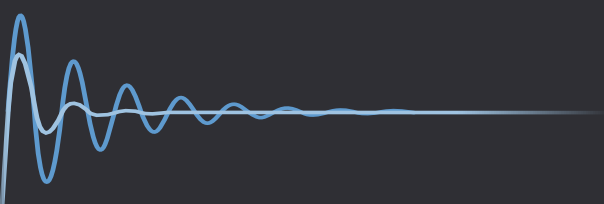

Wednesday November $28^{\text {th }}, 2012$

14:30 Introductory talk

14:45 Start of defense

16:00 Reception

The Waaier building, room 4

University of Twente

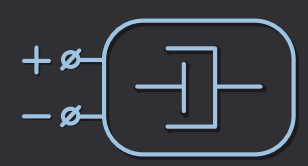

Bayan Babakhani Asbreukerhoek 2 7546 CR Enschede $06-54930029$ bayan.babakhani@gmail.com

Paranymphs: Stephanie Mossel

Roonak Babakhani 